\title{
The Dynamics of Cognition: An ACT-R Model of Cognitive Arithmetic
}

\author{
Christian Lebiere \\ November 23, 1998 \\ CMU-CS-98-186 \\ School of Computer Science \\ Computer Science Department \\ Carnegie Mellon University \\ Pittsburgh, PA \\ Submitted in partial fulfillment of the requirements \\ for the degree of Doctor of Philosophy \\ Thesis Committee: \\ John R. Anderson, Chair \\ Bonnie John \\ Tom Mitchell \\ Robert Siegler, Psychology Department, Carnegie Mellon University \\ (C) 1998 Christian Lebiere
}

This research was sponsored by the Office of Naval Research under contract number N00014-95-10223 to John Anderson at Carnegie Mellon University. The views and conclusions contained in this document are those of the author and should not be interpreted as representing the official policies, either expressed or implied, of the ONR or the U.S. government. 
Keywords: ACT-R, cognitive arithmetic, Bayesian learning, activation spreading, dynamical systems, parameter analysis, power law, machine learning, hybrid systems. 


\begin{abstract}
Cognitive arithmetic, the study of the mental representation of numbers and arithmetic facts and the processes that create, access and manipulate them, offers a unique window into human cognition. Unlike traditional Artificial Intelligence (AI) tasks, cognitive arithmetic is trivial for computers but requires years of formal training for humans to master. Understanding the basic assumptions of the human cognitive system which make such a simple and well-understood task so challenging might in turn help us understand how humans perform other, more complex tasks and engineer systems to emulate them. The wealth of psychological data on every aspect of human performance of arithmetic makes precise computational modeling of the detailed error and latency patterns of cognitive arithmetic the best way to achieve that goal.

While specialized models have been quite successful at accounting for many aspects of cognitive arithmetic, this thesis aims to provide an integrated model of the field using a general-purpose cognitive modeling architecture (ACT-R). This model makes minimal assumptions but instead relies on the architecture's Bayesian learning mechanisms to derive the desired results from the statistical structure of the task. The behavior of this model is analyzed using several approaches: separate simulations of each main result, a single simulation of a lifetime of arithmetic learning, a formal analysis of the model's dynamics and an empirical variation of the simulation's parameters.

This thesis provides a unifying account of the main results of cognitive arithmetic. Through its parameter analysis, it suggests some practical lessons for the teaching of arithmetic. The constraints of a lifetime simulation of arithmetic learning also expose the underlying assumptions of ACT-R's associative learning mechanism. While a simplifying assumption commonly used in machine learning is shown in this case to be inadequate, a more powerful algorithm closely replicates human behavior. The formal and empirical analyses of the model parameters establish that despite its less-than-perfect performance, human cognition is surprisingly optimal. Finally, the behavior of the simulation through a lifetime of arithmetic learning can best be described as a dynamical system affected not only by its external environment but also by its internal dynamics.
\end{abstract}


Dedicated to the memory of my father, and the promise of my son. 


\section{Table of Contents}

CHAPTER 1: INTRODUCTION

CHAPTER 2: MODEL AND DATA

2.2 Model

2.2.1 Basic Representation and Productions

2.2.2 Sub-symbolic Processes

2.3.1 Problem Size Effect

2.3.2 Errors in Addition Retrieval

2.3.3 Errors in Multiplication Computation

3.2 Learning the Correct Answer

3.2.1 Overview

3.2.2 Basic Dynamics of Error Odds

3.2.3 Context and Complexity

3.2.4 Matching Penalty

3.2.5 Multiple Alternatives

3.2.6 External Feedback Sources

3.2.7 Comparison

4.3 Associative Strengths and Interference $\quad 56$

4.3.1 General Introduction $\quad 56$

4.3.2 Tie Problems

4.3.3 Near-tie Problems

4.4.4 Corner Problems

4.3.5 Cross-type Interference

4.3.6 A More General Solution 
5.2 Activation Noise

5.3 Retrieval Threshold

5.4 Mismatch Penalty

5.5 Presentation Schedule

5.6 Steepness of Frequency Distribution

5.7 Feedback Probability

5.8 Discussion

6.1 Feeling of Knowing

6.2 Strategy Choice

6.3 Multiplication Errors

CHAPTER 7: CONCLUSION

7.1 Difficulties

7.2 Contributions

TABLES

Table 1: Equations

Table 2: Symbols

Table 3: Parameters 


\section{Acknowledgments}

This thesis started with the support of my family, and their repeated encouragement that this was something worth doing. My wife Theresa provided her love and her relentless determination in getting things done. I will be forever thankful to her for putting up with me. My father-in-law ceaselessly sang the virtues of education. My son Mathieu was the source of endless distractions, and so many reminders that there are more important things too. I want to thank them all for their love and support.

The ACT-R group at Carnegie Mellon and beyond provided much of the intellectual background to this work. The discussions in the hallway, at the weekly Friday research meeting, and at the yearly ACT-R workshop were the testing-bench on which these ideas were refined. I want to thank in particular Dieter Wallach for those extraordinarily stimulating few months we spent as officemates. Science is indeed a cooperative endeavor.

I would like to thank my committee for guiding me and asking all the right questions. Tom Mitchell lent a benevolent but always insightful ear. Bob Siegler provided encyclopedic knowledge of the field. Ever since the first ACT-R summer school, Bonnie John has always asked the tough architectural questions, and never taken no for an answer.

Most importantly, I want to thank my advisor, John Anderson, for giving me this opportunity. He showed me how research is done and allowed me to be productive. Without him this thesis would never have seen the light of day. 


\section{Chapter 1: Introduction}

Cognitive arithmetic studies the mental representation of numbers and arithmetic facts (counting, addition, subtraction, multiplication, division) and the processes that create, access and manipulate them. Arithmetic is one of the fundamental cognitive tasks (one of the three basic "R"s) which humans have to master. Children go through years of formal schooling to learn, first the numbers, then the facts and skills needed to manipulate them. Many adults have not and will never completely master the domain. And yet it is a task that is trivial to perform correctly for computer architectures.

Some tasks, such as natural language processing or chess, are hard for both humans and machines to perform and require years of learning or engineering. Other tasks, such as vision, which seem to come naturally to humans, require much programming for computers to perform even poorly. One can attribute that to humans possessing a complex vision system which resulted from millions of years of evolution but will require painstaking work to reverse-engineer and replicate in computers. But a task such as arithmetic seems so straightforward and easy to accomplish that it is surprising that it takes years of learning for humans to master. This suggests that human cognition at the most basic level embodies some assumptions about its environment that are at odds with the structure of arithmetic as it is taught. Arithmetic, being a formal mathematical theory, assumes a set of precise and immutable objects (the numbers), facts, and procedures. Human cognition, on the other hand, has evolved to deal with approximate concepts, changing facts, and adaptive procedures. Studying how such a system deals with a formal task such as arithmetic provides an excellent window to its assumptions and mechanisms.

ACT-R is a hybrid production-system theory of human cognition (Anderson, 1993; Anderson \& Lebiere, 1998). At the symbolic level, ACT-R is a fairly standard goaldirected production system, with a declarative memory of long-term facts, known as 
chunks, and a procedural memory holding general production rules. At that level, cognitive arithmetic is a trivial task for ACT-R. All one needs to do is give ACT-R the correct chunks representing arithmetic facts and productions encoding procedures to manipulate them and perfect performance will result. This, however, ignores the impact of ACT-R's sub-symbolic level and would not be a very satisfactory model of human, especially children's, performance. ACT-R is also an activation-based system in which the performance at the symbolic level is controlled by associated real-valued quantities. Those quantities are learned according to Bayesian principles to reflect the architecture's environment. Retrieval and matching of memory chunks by production rules is a noisy, approximate process driven by activation rather than the exact matching of conditions. Thus the behavior of the system becomes adaptive, stochastic and error-prone, matching human behavior better but making cognitive arithmetic a more challenging, but also more interesting task.

Cognitive arithmetic is a task that is both well suited and challenging to ACT-R for a number of reasons. Unlike tasks artificially designed for the purpose of isolating a particular cognitive mechanism, the learning and performance of arithmetic involves almost every mechanism of the architecture. It is therefore an excellent test of whether these parts can perform together as well as separately. Unlike laboratory tasks, large amounts of data are available for every cross-section of the population and every aspect of the task, making it easier to establish the trends being analyzed.

While numbers can be seen as having a concrete interpretation (e.g. children learn the concept "three" by being shown three rabbits), the rest of arithmetic has essentially an abstract structure. It is much less likely that people have brain structures optimized to perform arithmetic than for example vision or language, and suggests a complete reliance on general-purpose learning mechanisms. Since each skill builds on the previous ones, e.g. counting can be used to perform addition, which in turn can be used to perform multiplication, learning can thus be a mostly self-contained process, rather than entirely dependent upon external factors such as teaching. Arithmetic also has an inherently clear, simple and regular structure, with a systematic organization of knowledge into 
tables of immutable facts. This strong regularity, unlike for example the many exceptions of tasks such as natural language processing, also helps in reducing degrees of freedom in modeling the task and provides a good test of ACT-R's statistical learning. These factors lead to a simpler, more regular model that is more predictive than one with many unanalyzed degrees of freedom.

There are two classes of empirical phenomena in the domain of arithmetic that any model needs to account for. One concerns the fact that children, and to a certain degree adults, approach answering arithmetic problems with two basic strategies. One strategy is to simply retrieve the answer. The second strategy, referred to hereafter as the backup strategy or backup computation, is to compute the answer. Thus, given a problem such as $3+4=$ ? children may choose to count (perhaps $4,5,6,7)$ to provide the answer and given $3 * 4=$ ? they may choose to add to get the answer (perhaps $4+4+4$ ). One class of empirical phenomena involves how people choose between the computation strategy and the retrieval strategy.

The second class of empirical phenomena involves the problem-size effect. Children and adults take longer to answer problems involving larger numbers and they also make more errors on these problems. In the case of backup computation the reason for this is fairly obvious -- one has to count more to add large numbers and one has to add more things when multiplying by a larger number. However, while much reduced, the problem-size effect occurs for adults. It has been suggested that this is due to residual use of the backup strategy (LeFevre, et. al., 1996a), although recent research put those results in doubt (Kirk \& Ashcraft, 1997). However, it has been argued that smaller problems also occur more often, offering greater practice. This is true in studies of textbooks (Ashcraft, 1987; Ashcraft \& Christy, 1995; Hamman \& Ashcraft, 1986; Siegler, 1988) but it is also true in the world at large. As many (Benford, 1938; Newcomb, 1888; Raimi, 1976) have noted, small numbers occur more often in the world generally. As just one interesting token of the ubiquity of small numbers, consider the addition problems created by adding the two rows in multiplication problems involving two-digit numbers. An example is given below: 
The problem creates a $3+8$ addition problem and a $1+6$ addition problem. If one looks at all such multiplication problems with multiplicands from 10 to 99 , one finds that addition problems involving smaller addends occur more frequently. Figure 1.1 plots, as a function of the size of the addend, the frequency of all additions problems created by adding the tens digit from the top row (i.e. 138) with the ones digit from the bottom row (i.e. 368) or the hundreds digit from the top row with the tens digit from the bottom row. There is a clear drop off with size of the addend. ${ }^{1}$

\section{1-Digit Addition from 2-digit Multiplication}

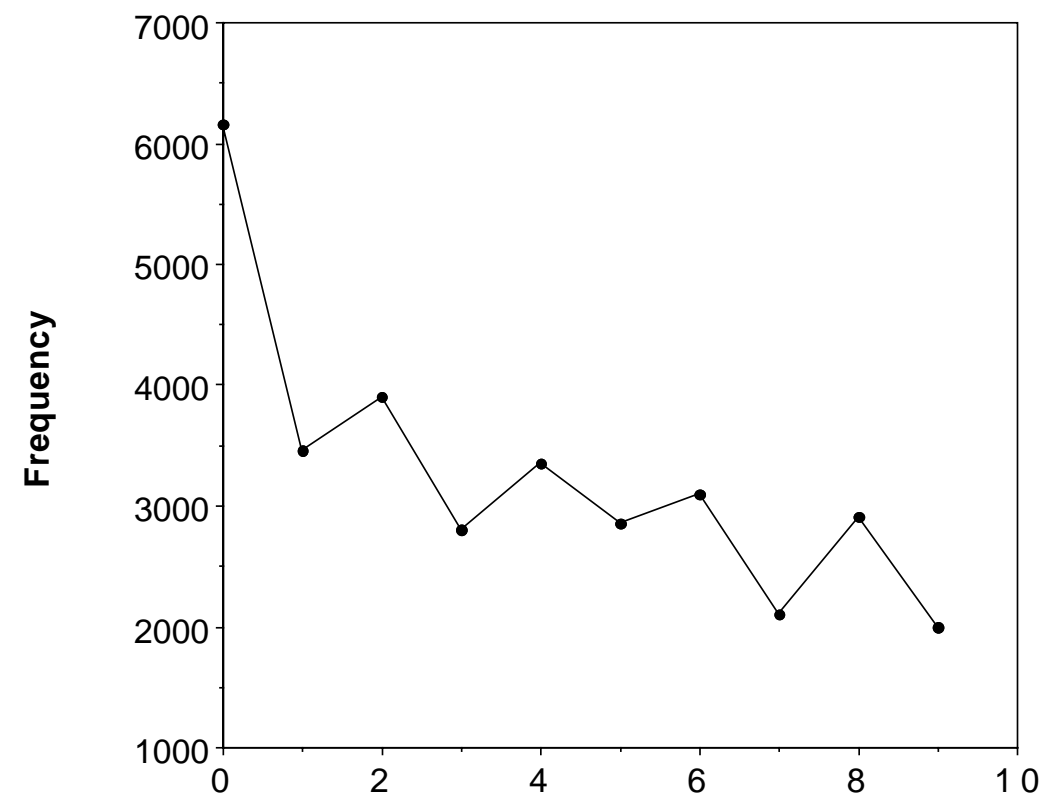

Addend

Figure 1.1: Frequency of 1-digit Addition Problems Created by 2-digit Multiplication Problems.

\footnotetext{
${ }^{1}$ The drop-off with size of addend on Figure 1.1 happens because the hundreds digit in these addition problems tends to be small and the saw-tooth pattern is produced because the ones digit is more often even.
} 
These effects of problem size and strategy are ubiquitous throughout the literature on cognitive arithmetic (for reviews of the field, see e.g. Ashcraft, 1992, 1995; Campbell, 1995; Geary, 1996). While these effects are not by far the only ones to account for, they constitute a good basis for a comprehensive model of cognitive arithmetic. In a model of Zbrodoff's alphabet arithmetic experiment (Anderson \& Lebiere, 1998), it was shown that ACT-R had the ability to account for these effects in the miniature. However, it is another question whether, when ACT-R's learning mechanisms are turned on and given a lifetime of experience, the resulting behavior will look at all like human behavior. This is the challenge that this thesis is trying to address.

Chapter 2 describes the basic model and its ability to account for basic results in the field of cognitive arithmetic. These demonstrations will be typical "mini-models" which assume a certain distribution of knowledge strength at a particular point in time. Chapter 3 analyzes how to go beyond those limited models in creating a model that gradually improves its performance with practice. Chapter 4 presents such a model, called the lifetime simulation, which like human students goes through the equivalent of years of training while replicating the results that were previously modeled separately. From the difficult constraints imposed by the lifetime simulation will be derived a number of lessons for the use and evolution of the architecture. Chapter 5 analyzes the sensitivity of the lifetime simulation to its parameters and draw conclusions regarding the nature of human cognition and the teaching of arithmetic. Chapter 6 discusses some issues concerning cognitive arithmetic, including the feeling of knowing, the question of strategy selection and the nature of human guessing. Chapter 7 summarizes the difficulties encountered in this endeavor and the contributions made by this thesis. 


\section{Chapter 2: Model and Data}

\subsection{ACT-R}

ACT-R is an activation-based goal-directed production system theory (Anderson, 1993; Anderson \& Lebiere, 1998). Knowledge in ACT-R is divided into declarative knowledge stored in chunks (e.g. arithmetic facts) and procedural knowledge stored in productions (e.g. how to retrieve a fact given a problem). Sub-symbolic activation processes control which productions are used and how they apply to chunks. The parameters of these numerical processes reflect the past statistics of use of the knowledge structures to which they are attached. They are learned by Bayesian learning mechanisms derived from the rational analysis of cognition (Anderson, 1990). The equations governing this subsymbolic level will be described below and are summarized in Table 1 and 2 .

In ACT-R, the activation of a declarative memory element, or chunk, can be interpreted as reflecting the log posterior odds that the chunk is relevant in the current context. The activation of a chunk is the sum of the base-level activation of that chunk plus the sum for all context elements of their attentional weights (a.k.a. activation source level) times the strength of association between the context element and the chunk. In Bayesian terms, the base-level activation represents the log prior odds of the chunk being relevant and the strength of association represents the log likelihood ratio that the chunk is relevant given the context element.

Formally, the activation $A_{i}$ of chunk $i$ is defined as:

$$
A_{i}=B_{i}+\sum_{j} W_{j} S_{j i}
$$

\section{Activation Equation}

where $B_{i}$ is the base level activation of $i, W_{j}$ is the attentional weight given the focus 
element $j$, and $S_{j i}$ is the strength of association (a.k.a. Interactive Association or IA values) from element $j$ to chunk $i$. An element $j$ is in the focus, or in context, if it is a part of the current goal, and the total amount of attention is divided evenly among goal elements.

The base level activation of a chunk can be learned to reflect the past history of use of that chunk:

$$
B_{i}=\ln \sum_{j=1}^{n} t_{j}^{-d}
$$

\section{Base-Level Learning Equation}

where $t_{j}$ is the time elapsed since the $j$ th occurrence (retrieval) of chunk $i, n$ is the total number of references to that chunk and $d$ is the memory decay rate. With the assumption that references are evenly distributed, the previous equation reduces to a simpler form that can be more efficiently computed:

$$
B_{i}=\ln \frac{n \cdot L^{-d}}{1-d}
$$

\section{Optimized Learning Equation}

where $L$ is the lifetime of the chunk, i.e. the time since its creation.

Similarly, the strengths of associations can be learned to reflect the past history of use of a chunk given its context:

$$
S_{j i}=\ln \frac{\operatorname{assoc} \cdot R_{j i}^{*}+F\left(C_{j}\right) \cdot E_{j i}}{\operatorname{assoc}+F\left(C_{j}\right)} \quad \text { Posterior Strength Equation }
$$

where $R_{j i}{ }^{*}$ is the prior strength of association, assoc is the weight given to that prior, $F\left(C_{j}\right)$ is the frequency of $j$ being in the context (i.e. a source of activation in the goal), and $E_{j i}$ is 
the empirical strength of association. Initially, the strength of association is 0 if the source $j$ does not appear as a slot value in chunk $i$, and otherwise is equal to:

$$
\ln \left(R_{j i}^{*}\right)=\ln (m / n) \quad \text { Prior Strength Equation }
$$

where $m$ is the total number of chunks in declarative memory and $n$ is the number of chunks which contain the source chunk $j$. Their ratio is a static estimation of the increased likelihood of retrieving chunk $i$ when chunk $j$ is a source of activation. With extensive experience, the strength of association converges to:

$$
\ln \left(E_{j i}\right)=\ln \left(\frac{F\left(N_{i} \& C_{j}\right) \cdot F}{F\left(N_{i}\right) \cdot F\left(C_{j}\right)}\right)
$$

\section{Empirical Ratio Equation}

where $F\left(N_{i} \& C_{j}\right)$ is the frequency of chunk $i$ being needed (retrieved) with chunk $j$ in context, $F\left(N_{i}\right)$ is the frequency of $i$ being needed, $F\left(C_{j}\right)$ is the frequency of $j$ being in the context (i.e. a source of activation in the goal), and $F$ is the total number of opportunities (productions matched) since $i$ was created.

In exact matching mode, ACT-R only considers the chunks that match perfectly the production condition(s). In partial matching mode, every chunk of the correct type is considered, but a mismatch to the production condition results in a penalty being subtracted from the chunk activation to yield its match score:

$$
M_{i p}=A_{i}-M P \cdot \sum(1-\operatorname{Sim}(v, d)) \quad \text { Match Equation }
$$

where $M P$ is the mismatch penalty constant and $\operatorname{Sim}(v, d)$ is the similarity between the desired slot value $d$ specified in the production condition and the actual slot value $v$ contained in the chunk. Gaussian noise of mean 0 and standard deviation $\sigma$ is also added to the activation and the chunk with the highest final match score is then selected, 
assuming that it reaches the retrieval threshold $\tau$. If one approximates the Gaussian noise with a sigmoid distribution, the probability $P$ of chunk $i$ being retrieved by production $p$ is:

$$
P=\frac{1}{1+e^{-\frac{M_{i p}-\tau}{s}}}
$$

\section{Retrieval Probability Equation}

where $s=\sqrt{ } 3 \sigma / \pi$.. If no chunk reaches the retrieval threshold, then a retrieval failure occurs and the next production is selected. If more than one chunk is competing for retrieval, the probability $P(i)$ of chunk $i$ being the one that is retrieved follows the Boltzmann equation, i.e.:

$$
P(i)=\frac{e^{M_{i p} / t}}{\sum_{j} e^{M_{j p} / t}}
$$

\section{Chunk Choice Equation}

where $t=\sqrt{ } 2 s$. The latency Time $_{i p}$ to retrieve (match) a chunk $i$ with production $p$ is an exponentially decreasing function of the sum of the chunk's match score and the production strength $S_{p}$ (which, like a chunk's base level, is a reflection of the frequency of use of the production):

$$
\text { Time }_{i p}=F e^{-f\left(M_{i p}+S_{p}\right)}
$$

\section{Retrieval Time Equation}

where $F$ is a time scaling constant and $f$ an activation scaling constant usually left to its default value of 1 (i.e. ignored). The productions that can apply to the current focus of attention are matched in sequence by decreasing value of expected gain $E$ : 
where $G$ is the value of the current goal, $P$ is its ultimate probability of success given this production firing and $C$ is the cost of execution of this and following productions until completion of the goal. Gaussian noise is added to the expected gain value, and thus production selection will also be stochastic and follow the Boltzmann distribution. The probability and cost parameters can also be learned according to the record of success and failure of each production. The reader should consult Anderson \& Lebiere (1998) for additional details of the ACT-R theory. More information about ACT-R, including the models that are presented in this thesis, is available on the ACT-R web site at http://act.psy.cmu.edu.

\subsection{Model}

This section will set forth the basic model of cognitive arithmetic. There is nothing particularly novel in the types of chunks and productions that were chosen. They reflect a common approach in the ACT-R community and are already used to model many phenomena.

\subsubsection{Basic Representation and Productions}

Arithmetic problems are represented as chunks with four slots: one for the operator, one for each operand and one for the result. For example, the chunk representing the fact that $2+3=5$ would be:

\section{Fact $-2+3=5$}

isa arithmetic

first 2 operator + second 3 result 5 
where $2,+, 3$ and 5 are other chunks representing the numbers and operator. ${ }^{2}$ The most basic action that one can perform on knowledge chunks is to retrieve them. This is accomplished by the Retrieval production, which solves an arithmetic problem by simply retrieving the answer to the problem stored in long-term memory:

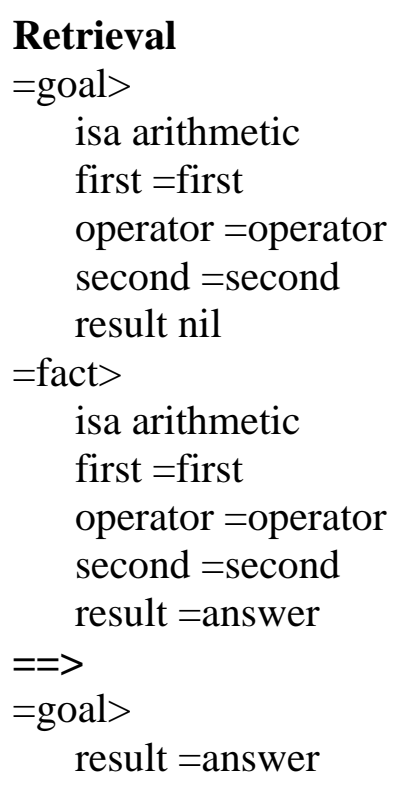

This production simply retrieves a chunk (fact) matching the goal (problem), then copies the answer back to the goal. One can notice that the chunk retrieved from memory is of the same type as the goal representing the problem, and wonder how the fact was initially created. In ACT-R 4.0, there are only two possibilities. The first is that it results from the encoding of an environmental stimulus. In this case, this would correspond to an external source of arithmetic knowledge such as a teacher, a table from a book, or a calculator. The second possibility is the long-term encoding of a past goal. If one cannot retrieve a fact one can (re)generate the arithmetic knowledge by the use of backup computation strategies. An example of such a strategy, which is to perform an addition by repeatedly counting up from one argument a number of times equal to the second argument, can be implemented by the production Iteration:

\footnotetext{
${ }^{2}$ Although addition and multiplication are commutative operations, this is not reflected in the declarative representation of facts, i.e. $2+3=5$ and $3+2=5$ are represented as separate chunks. Of course, this does not prevent explicit procedures to exploit the inherent commutativity, e.g. solve the problem $2+3=$ ? by retrieving the fact $3+2=5$.
} 


$$
\begin{aligned}
& \text { Iteration } \\
& \text { =goal }> \\
& \quad \text { isa arithmetic } \\
& \text { first }=\text { first } \\
& \text { operator }+ \\
& \text { second =second } \\
& \text { result nil } \\
& ==> \\
& =\text { subgoal }> \\
& \quad \text { isa iterate } \\
& \quad \text { result =first } \\
& \quad \text { counter } 0 \\
& \text { limit =second } \\
& \text { increment } 1 \\
& \text { result =answer } \\
& \text { !push } !=\text { subgoal } \\
& =\text { goal }> \\
& \quad \text { result =answer }
\end{aligned}
$$

This production solves an addition problem by setting a subgoal to iteratively add the second argument to the first by increments of 1, using the basic counting skills. Table 2.1 shows the two production rules that were used to accomplish this iterative counting procedure. This counting subgoal is pushed on the stack, and its result will be returned to the current goal as the answer to the problem using the subgoal value return mechanism. When an answer to the problem has been found using either retrieval or one of the backup strategies, the answer is output and the goal is popped by the Answer production:

$$
\begin{aligned}
& \text { Answer } \\
& \text { =goal> } \\
& \text { isa arithmetic } \\
& \text { first }=\text { first } \\
& \text { operator =operator } \\
& \text { second =second } \\
& \text { result =answer } \\
& ==> \\
& \text { !output! =answer } \\
& \text { !pop! }
\end{aligned}
$$

When the goal is popped, it becomes a fact in long-term memory. If this fact did not already exist, then the solving of this problem (presumably using the backup strategies) has added a new arithmetic fact to the knowledge base. If an identical fact already 
existed (modulo the chunk name ${ }^{3}$ ), then the new chunk is merged with the existing one, reinforcing it, and the duplicate copy is removed from declarative memory. If the problem could not be solved by retrieval, this reinforcement from the merging with the new problem will raise the activation of the fact until ultimately the problem can be solved by retrieval. If the problem was already solved by retrieving the fact, then it will receive two learning reinforcements: first, from its use in the retrieval production and, second, from being merged with the problem goal.

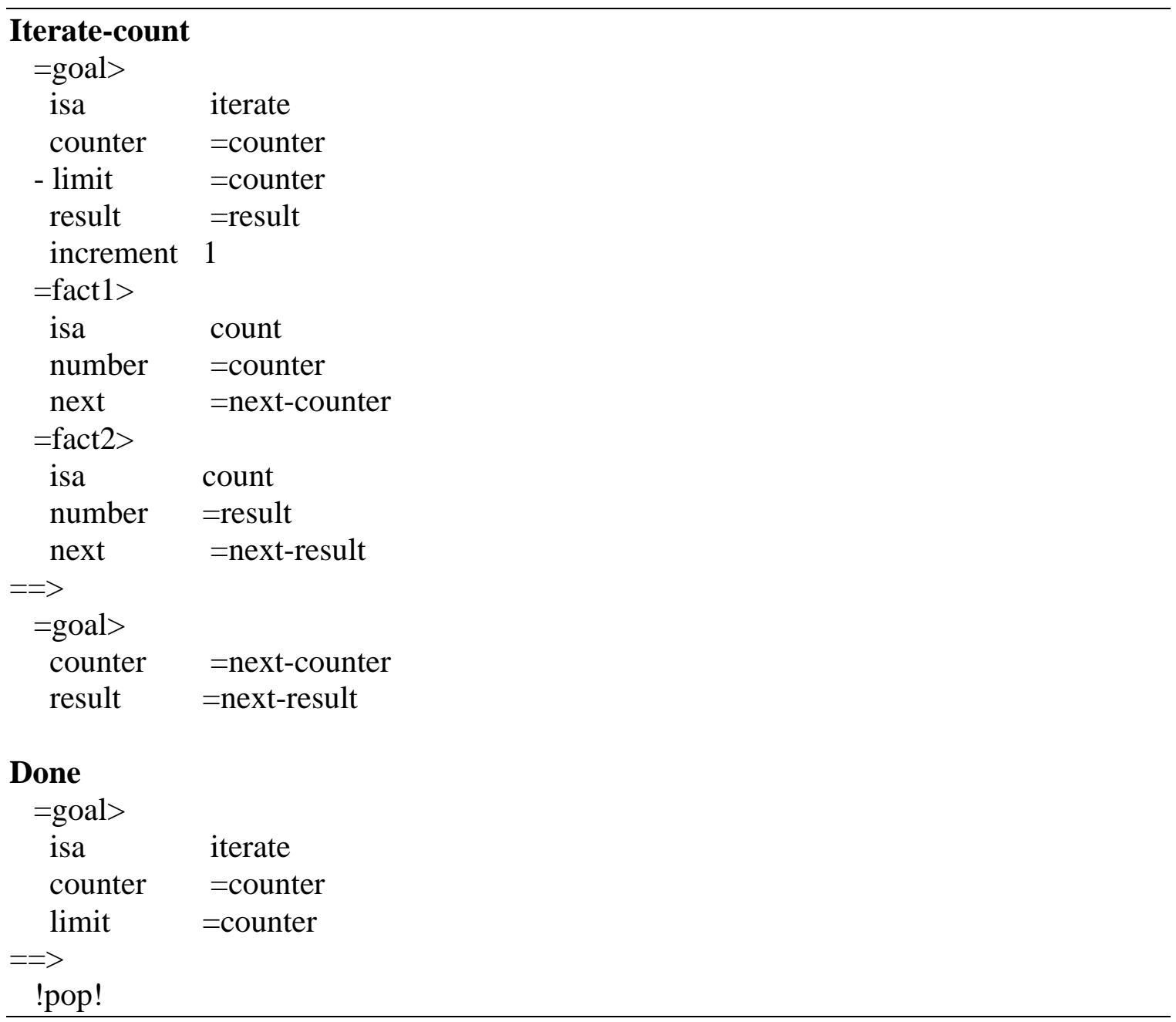

Table 2.1: Productions for Addition by Iterative Counting.

\footnotetext{
${ }^{3}$ This, together with the rather dubious meaning of whichever name happens to be associated with such facts, suggests that chunk names are superfluous and that chunks could be best understood as the content of their slots rather than referred to by name.
} 


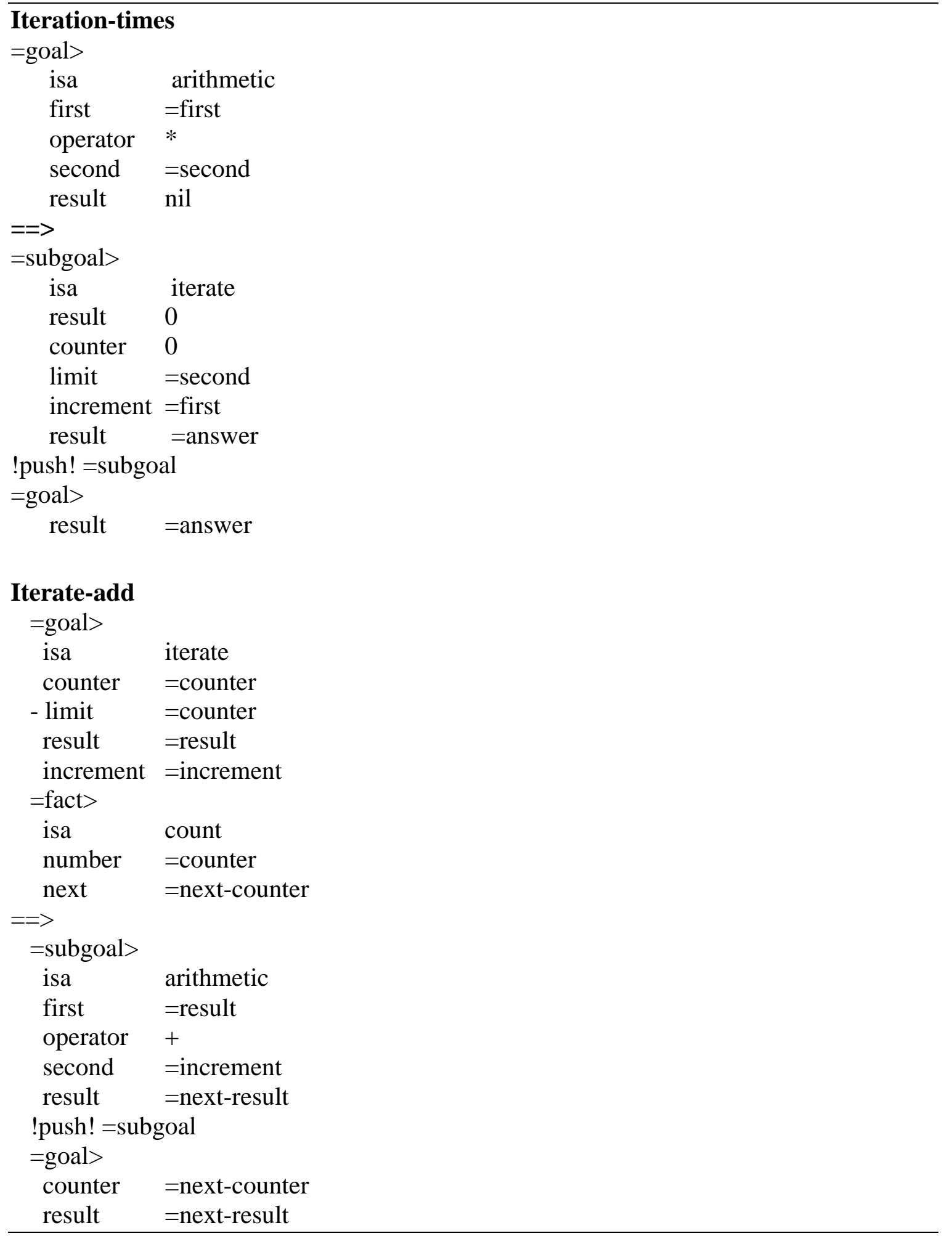

Note: The Retrieval and Done productions are the same as for addition.

Table 2.2: Productions for Multiplication by Iterative Addition. 
Since past goals are the only source of chunks (other than for environmental encoding), this technique of solving a problem by pushing a goal which can be solved either by directly retrieving the answer from the corresponding fact or by using a number of backup strategies is a general ACT-R technique to model problem-solving. By gradually raising the activation of the necessary facts with practice, it provides a general account of the transition from general problem-solving strategies toward more efficient ones. As noted in (Anderson \& Lebiere, 1998), ACT-R implements Logan's (1988) proposal for transition from algorithmic solutions to direct retrieval.

The discussion has focused on addition but a parallel model for multiplication has been developed. The iterative addition procedure, corresponding to the counting procedure, is given in Table 2.2. The productions there try to retrieve the multiplication answers and if they fail call on a backup strategy of repeated addition.

\subsubsection{Sub-symbolic Processes}

Since the retrieval and iteration productions (and possibly other backup strategies) share the same goal condition, conflict resolution is needed to determine which productions are fired in which order. Typically (and this may not be true for, say, small children), the retrieval production provides a high probability of producing a correct answer at low cost, and thus will have the highest evaluation and will be attempted first. If no arithmetic fact for that problem is above threshold, the retrieval production will time out and the next production in the conflict resolution order, e.g. iteration, will be allowed to fire.

A general observation is that children will choose to retrieve more often for smaller problems and choose to compute more often for larger problems (Siegler, 1988). The simplest explanation for this in ACT-R is that subjects cannot retrieve the answer in the case of large problems and fall back on computation. This would occur more often for larger problems because they have less practice (e.g., Figure 1.1). Of course, for people with a poor knowledge of the facts and a fairly reliable backup strategy, the expected gain of the retrieval production might well be lower than the expected gain for the backup 
production(s). In general, people may evolve complex sets of strategies for making the decision between retrieve and compute. However, let us ignore these complications and simply assume that the two strategies are retrieve and compute. Moreover, to the extent that retrieve is preferred in conflict resolution, subjects will only choose to compute after they have failed to retrieve the answer. This preference for retrieval as the first way of solving the goal can be seen as an instance of the Obligatory Retrieval Assumption of Logan (1988), i.e. people cannot help but try to retrieve the answer to an arithmetic problem and only resort to explicit computation when that fails. Those issues will be examined in detail in Chapter 6.

Clearly, the activation of chunks storing arithmetic facts is going to be very critical to ACT-R's performance in cognitive arithmetic. The activation of a chunk is given as a sum of a base-level activation and associative activation according to the Activation Equation. The base-level activation will change with experience according to the BaseLevel Learning Equation in such a way that it grows approximately as a log function of amount of practice. The strengths of association will change with experience according to the Posterior Learning Equation such that it will come to vary approximately as a log function of the odds of a chunk being retrieved when another is in the context.

These activation quantities are converted into match scores that reflect the effects of partial matching (Match Equation). In the case of a perfect match, the match score is just the activation but in the case of a mismatch a penalty will be subtracted from the match score. There is noise in these match scores because of activation noise. If the match score is above a threshold the chunk will be retrievable and the probability of it being retrieved (rather than some other chunk) is described by the Retrieval Probability Equation. If there are multiple possible chunks that might match the one chosen is the one with the highest match score and the probability of any one being chosen is described by the Chunk Choice Equation. Finally, match scores determine latency through the Retrieval Time Equation.

Errors can be committed whether the subject is computing or retrieving. Let us consider 
the example of the problem $2+3=$ ?. Because of ACT-R's partial matching process it is possible for ACT-R to retrieve an arithmetic chunk (e.g. $2+4=6$ ) other than the correct one. Recall that chunks are retrieved on the basis of their match scores that are calculated as their activation levels minus mismatch scores. It is possible that even after the mismatch score is subtracted off, the wrong chunk will have the highest match score and be retrieved instead and its answer stored in the current goal. In this model, the mismatch penalty between numbers increases linearly as a function of the difference between the two numbers. Thus, the mismatch penalty between numbers $i$ and $j$ is $D *|i-j|$ where $D$ is a scale factor to be estimated. The mismatch measure essentially encodes the representational similarity between numbers. ${ }^{4}$ This assumption about the representation of numbers has also been adopted in a number of other models of numerical memory (Anderson, Spoher \& Bennett, 1992; Campbell, 1995; McCloskey \& Lindemann, 1992).

Errors can also occur using the backup procedure when the iteration subgoal returns an erroneous answer because of a misretrieval, a procedural error or any other reason. The erroneous answer will also be stored in the goal. In both cases of retrieval and computation errors, not only will the answer to this particular problem be wrong, but the goal holding the incorrect answer is popped and becomes an erroneous long-term fact (here, $2+3=6) .^{5}$ This fact can then be retrieved as the answer to future problems and perpetuate the error. This otherwise correct retrieval of an erroneous fact becomes another source of error. This competition between memories for both correct and erroneous answers is quite similar to Siegler's treatment (e.g. Siegler, 1988). It might seem possible that ACT-R could reach an unfortunate state where it has so practiced the wrong facts that it comes to believe them. Indeed this can occur and the next chapter describes what must be true for ACT-R to avoid getting absorbed into such error states.

\footnotetext{
${ }^{4}$ Past models have been relatively insensitive to the exact form of the mismatch measure, but Whalen (1996) argues that the internal representation of numerical magnitude is not uniform and influences performance of numerical tasks. The lifetime simulation uses such a measure where similarities are proportional to the ratio between numbers instead of their difference.

${ }^{5}$ This assumes that error correction, provided by another procedure, a teacher or a
} 


\subsection{Data}

This section will examine how this ACT-R model can account for a wide range of effects in cognitive arithmetic, including the problem-size effect and the patterns of errors in retrieval and computation of addition and multiplication problems. Even though these effects typically have multiple, complex sources, simplifying assumptions were made for the sake of analysis. The lifetime simulation will eliminate the simplifying assumptions and account for these effects in a more complex manner. The basic import of the results presented here is that even a fairly simple approach can successfully account for those effects. The methodology will be as follows: given a data set describing the performance of human subjects at a particular point in life (e.g. 4-year-olds, $4^{\text {th }}$ graders, adults) on a particular task (e.g. addition retrieval, multiplication computation) for a particular measure (e.g. latency, error percentage), assumptions will be made about the distribution of knowledge at that point in time (e.g. amount of practice) and about the value of the simulation parameters, and the relevant part of the model (e.g. if the task is retrieval, then the computation productions will not be used) will be run to provide the model's predictions. Again, the lifetime simulation will model all those data sets in a single simulation with the same set of parameters and assumptions. The parameters used in those simulations are summarized in Table 3.

\subsubsection{Problem Size Effect}

The most basic and robust effect in cognitive arithmetic is that larger problems are harder. This holds for measures of retrieval time and error rates, for the four basic operations, for production and verification tasks, and for the entire age span from children to adults and elderly (e.g. Ashcraft, 1992). Ashcraft (1987) reports the change in response time for addition problems in adults. Figure 2.1 illustrates the relationship between the sum of the digits and retrieval time. While most problems exhibit an increase in response time roughly corresponding to the square of the sum of their operands, the slope for problems involving a zero operand (squares in the graph) is

calculator, does not take effect before the goal is popped and becomes a long-term fact. 
approximately flat, and the increase in response time for tie problems (those having identical operands - triangles in the graph) is much smaller than for non-zero, non-tie problems (circles in the graph). The effect therefore reflects a more complex measure of problem difficulty than simply problem size.

\section{Problem Size Effect}

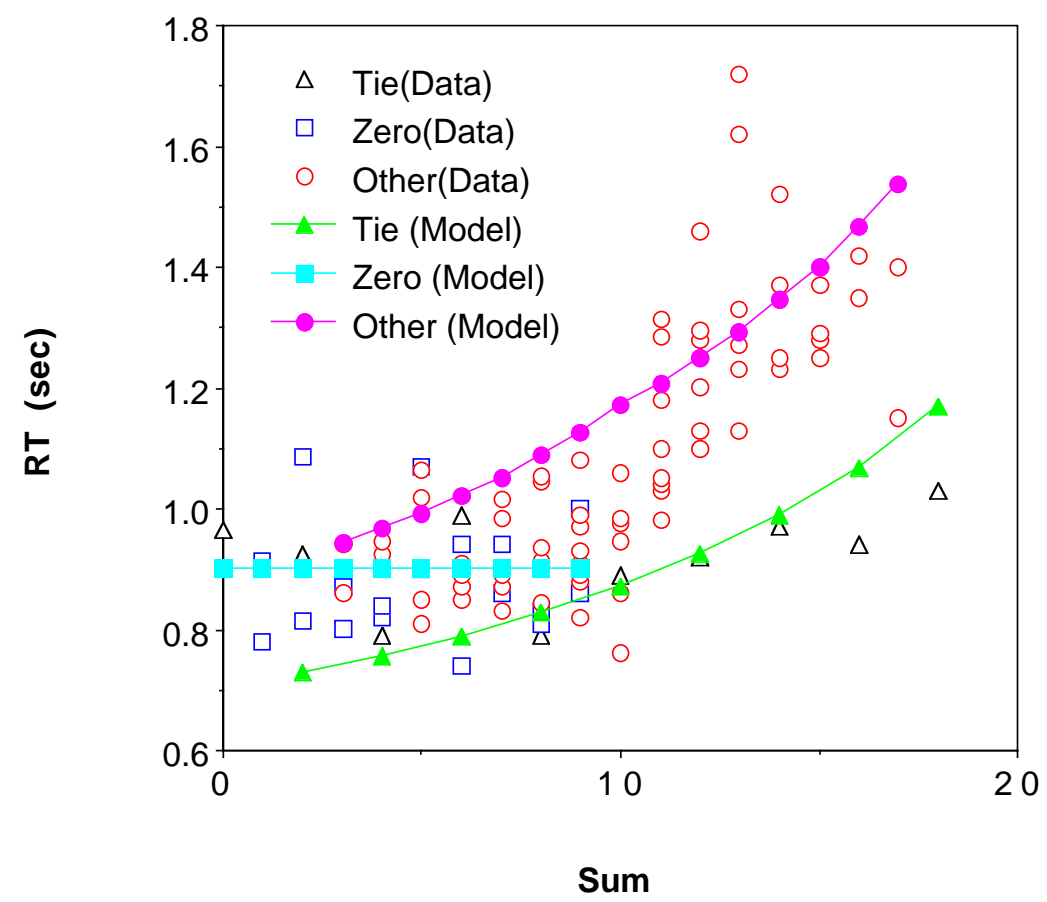

Figure 2.1: Problem Size Effect for Addition for Adults: The data are the open points and the predictions of ACT-R are the closed points connected by lines.

The flat response time for problems involving zero is usually assumed to be the result of a special-purpose rule for those problems (" $0+\mathrm{x}=\mathrm{x}=\mathrm{x}+0$ for all $\mathrm{x}$ "). This will be modeled by the use of the special Zero production. Two main explanations have emerged to account for the rest of the data. Groen and Parkman (1972) initially argued that the problem size effect resulted from the use of backup strategies such as iterative counting. Larger problems involve more counting and therefore higher latencies and more errors. If the first number is always added to the second (or vice versa), then the latency will increase linearly with the sum of the numbers. A better fitting model, called the min model, assumes instead that the smaller number is always added to the larger one, thereby minimizing the number of increments necessary. While this model certainly explains 
part of the problem size effect for children and other poorly trained subjects, it has difficulties in fully accounting for the effect in well-trained adults as well as the better performance on tie problems.

The other category of models relies on the difference of presentation frequency between problems. As was noted earlier, smaller problems occur more frequently than larger ones. Smaller problems are therefore easier because they are presented and practiced more often. Ashcraft (1987) presents the frequency of presentation of addition facts by operand in grades K to 3 and Siegler (1988) presents the frequency of multiplication problems in second- and third-grade workbooks. In each case the frequency decreases roughly linearly with operand size, except for very small operands which are special cases. It is generally assumed that the distribution in schoolbooks approximately reflects the real-life problem distribution. This frequency information was used in an ACT-R simulation whose results are illustrated as lines in Figure 2.1. The ratio of frequencies of the smallest $(0+0)$ to the largest $(9+9)$ was set at 4-to-1 and intermediate problems had a frequency that varied linearly with each operand. Thus, if $0+0$ occurred four times, $0+9$ and 9+0 would occur twice, and 9+9 would occur once. ${ }^{6}$ This distribution approximates closely the occurrence frequency in textbooks as described by Hamman and Ashcraft (1986). This simulation assumes that five hundred thousand problems were presented according to these frequencies at an average of a hundred problems a day. The underlying equation determining latency, based on the Retrieval Time Equation, is:

\section{Time $=I+F e^{-A}$}

where $I$ is an intercept reflecting encoding/answering times, $F$ is the latency factor from the Retrieval Time Equation, and $A$ is the activation of the chunk encoding the addition fact. The activation was determined by the half-million trials of experience in ACT-R. In the model $I$ was estimated at 0.4 second and $F$ was left at the default value of 1.0. An additional latency of 0.5 second was also estimated for the Zero production. As can be

\footnotetext{
${ }^{6}$ The relative frequency of a problem involving digits $i$ and $j$ was $(2-i / 9)(2-j / 9)$.
} 
seen in Figure 2.1 the model does a pretty good job of capturing the effects in the data. The basic increase in latency with problem size comes from ACT-R's base-level learning. It follows from the simplified form of the Base-Level Learning and Retrieval Time Equations that retrieval time is a power function of frequency, and since frequency decreases roughly linearly with problem size, then the response time for arithmetic retrieval grows as a power function of problem size.

The retrieval time for the zero operand problems is constant at 0.9 second while it increases slowly for tie problems to about 1.15 second for the largest problem. Tie problems generate additional spreading activation in ACT-R because one of the arguments appears twice in the context. This can be explained by looking at the addition facts $3+3$ and $3+4$, and comparing the $S_{j i}$ values learned according to the Posterior Strength Equation from the number 3 to a tie arithmetic fact $(3+3=6)$ and a non-tie arithmetic fact (e.g. $3+4=7$ ). In this case $j$ is the number 3 and $i$ is the fact. Assuming for simplicity that the two facts $(3+3=6$ and $3+4=7)$ are equally frequently needed, then all the components of the equation for the two facts are equal except for $F\left(N_{i} \& C_{j}\right)$, which is double for the tie fact because 3 is twice in the goal context for each retrieval, resulting in $S_{j i}$ values larger by $\ln (2)$ for tie facts. This additional activation spread to tie facts will in turn result in a decrease of their retrieval latency. Thus, the advantage of tie problems is a parameter-free prediction of ACT-R's mechanisms for associative learning. ${ }^{7}$

This simple model largely relies on differential presentation frequencies to produce the problem size effect. As will be seen later, differential frequencies of rehearsal (small problems are retrieved and thus reinforced before larger ones) and backup strategies (recomputing larger facts is more error-prone than smaller ones) also contribute to the problem size effect. Finally, even for adults part of the effect may result from the residual use of non-retrieval procedures (LeFevre et al, 1996a, but see Kirk \& Ashcraft,

\footnotetext{
${ }^{7}$ It is also possible that subjects encode tie problems using a special representation to reflect their unusual character (data supporting this conclusion is presented by (Eliaser, Siegler, Campbell, \& Lemaire, 1997)), which would affect the activation calculus as well as matching procedures. Finally, tie problems are often assumed to appear more frequently than indicated by their size alone, although that is not used in the simulation.
} 
1997). As mentioned previously, the lifetime simulation will take all these factors into account and determine their relative importance.

\subsubsection{Errors in Addition Retrieval}

Table 2.3 presents the pattern of retrieval errors of addition facts by 4 -year-olds found by Siegler and Shrager (1984). The subjects were presented with addition problems ranging from $1+1$ to $5+5$ and were asked to just state what they thought the answer was, without resorting to any overt strategy such as putting up fingers or counting. The main effect, similar to the problem size effect, is an increase in errors for larger facts. The facts showing a comparatively low percentage of errors are those involving the operand 1 , tie problems, and problems where the first operand is larger than the second one. Erroneous answers also tend to be smaller than the correct answer.

\begin{tabular}{|r|rrrrrrrrrrrrr|}
\hline & 0 & 1 & 2 & 3 & 4 & 5 & 6 & 7 & 8 & 9 & 10 & 11 & other \\
\hline $1+1$ & 0 & 5 & 86 & 0 & 2 & 0 & 2 & 0 & 0 & 0 & 0 & 2 & 4 \\
$1+2$ & 0 & 0 & 9 & 70 & 2 & 0 & 4 & 0 & 0 & 7 & 2 & 2 & 5 \\
$1+3$ & 0 & 2 & 0 & 11 & 71 & 5 & 2 & 2 & 0 & 0 & 0 & 0 & 7 \\
$1+4$ & 0 & 0 & 0 & 0 & 11 & 61 & 9 & 7 & 0 & 0 & 0 & 2 & 11 \\
$1+5$ & 0 & 0 & 0 & 0 & 13 & 16 & 50 & 11 & 0 & 2 & 2 & 0 & 5 \\
\hline $2+1$ & 0 & 7 & 5 & 79 & 5 & 0 & 0 & 0 & 0 & 0 & 0 & 0 & 4 \\
$2+2$ & 2 & 0 & 4 & 5 & 80 & 4 & 0 & 5 & 0 & 0 & 0 & 0 & 0 \\
$2+3$ & 0 & 0 & 4 & 7 & 38 & 34 & 9 & 2 & 2 & 2 & 0 & 0 & 4 \\
$2+4$ & 0 & 2 & 0 & 7 & 2 & 43 & 29 & 7 & 7 & 0 & 0 & 0 & 4 \\
$2+5$ & 0 & 2 & 0 & 5 & 2 & 16 & 43 & 13 & 0 & 0 & 2 & 0 & 18 \\
\hline $3+1$ & 0 & 2 & 0 & 9 & 79 & 4 & 0 & 4 & 0 & 0 & 0 & 0 & 4 \\
$3+2$ & 0 & 0 & 9 & 11 & 11 & 55 & 7 & 0 & 0 & 0 & 0 & 0 & 7 \\
$3+3$ & 4 & 0 & 0 & 5 & 21 & 9 & 48 & 0 & 2 & 2 & 2 & 0 & 7 \\
$3+4$ & 0 & 0 & 0 & 5 & 11 & 23 & 14 & 29 & 2 & 0 & 0 & 0 & 16 \\
$3+5$ & 0 & 0 & 0 & 7 & 0 & 13 & 23 & 14 & 18 & 0 & 5 & 0 & 20 \\
\hline $4+1$ & 0 & 0 & 4 & 2 & 9 & 68 & 2 & 2 & 7 & 0 & 0 & 0 & 7 \\
$4+2$ & 0 & 0 & 7 & 9 & 0 & 20 & 36 & 13 & 7 & 0 & 2 & 0 & 7 \\
$4+3$ & 0 & 0 & 0 & 5 & 18 & 9 & 9 & 38 & 9 & 0 & 2 & 0 & 11 \\
$4+4$ & 4 & 0 & 0 & 2 & 2 & 29 & 7 & 7 & 34 & 0 & 4 & 0 & 13 \\
$4+5$ & 0 & 0 & 0 & 0 & 4 & 9 & 16 & 9 & 11 & 18 & 11 & 4 & 20 \\
\hline $5+1$ & 0 & 0 & 4 & 0 & 4 & 7 & 71 & 4 & 4 & 0 & 4 & 0 & 4 \\
$5+2$ & 0 & 0 & 5 & 20 & 2 & 18 & 27 & 25 & 2 & 0 & 2 & 0 & 0 \\
$5+3$ & 0 & 0 & 2 & 11 & 9 & 18 & 5 & 16 & 23 & 0 & 5 & 0 & 11 \\
$5+4$ & 0 & 0 & 0 & 0 & 11 & 21 & 16 & 5 & 11 & 16 & 4 & 0 & 16 \\
$5+5$ & 4 & 0 & 0 & 0 & 0 & 7 & 25 & 11 & 2 & 4 & 34 & 4 & 11 \\
\hline
\end{tabular}

Table 2.3: Retrieval Frequencies for 5x5 Addition Retrieval in 4-year-olds.

Since according to instructions the children were asked not to use any procedure other 
than retrieval, the computation productions in the model were disabled. Although guessing and other such procedures could be considered, the basic mechanism for producing an arithmetic error in ACT-R is the mistaken retrieval of another partially matching fact (see Match Equation). According to the Chunk Choice Equation, the probability of such commission errors is proportional to the scaled activation of the intruding facts relative to the correct fact. Since activation is related to frequency, the frequency difference between problems ${ }^{8}$ is therefore critical to explaining the patterns of errors. The other factor is that partial matching penalties will be smaller among similar addition facts.

In the case of the retrieval of addition facts, small sums (especially those involving 1, which can be reduced to the well-practiced skill of counting) are practiced at a higher frequency and are therefore more likely to intrude upon another problem, leading to an error for that problem, than to be intruded upon. This higher activation for smaller facts also explains why the errors for larger facts tend to be biased toward numbers smaller than the correct answer. Tie problems receive an additional amount of activation, as described in the previous section, and are therefore more likely to be retrieved correctly. Finally, let us assume a small probability that, given a problem where the first operand is smaller than the second one (e.g. 2+4), students reverse the order of arguments to simplify counting (the min strategy) and therefore also rehearse the reverse answer $(4+2=6)$, giving it an advantage. The results are shown in Table 2.4. The model generates answer probabilities that are very close to the data.

This model assumes a thousand problem presentations (an average of 40 for each of the 25 problems in Table 9.3) with a distribution frequency ratio (between smallest and largest problems) of $6.25^{9}$, an activation noise $s$ parameter of 0.15 , a scaling mismatch penalty factor of .15 per digit difference ${ }^{10}$ and a retrieval threshold ${ }^{11} \tau$ of -2.25 . The

\footnotetext{
${ }^{8}$ Again, this difference can arise from presentation, rehearsal, and computation processes.

${ }^{9}$ A ratio of 6.25 rather than 4 as in the previous simulation was used to reflect the assumption that young children have a steeper distribution of frequencies than do adults. ${ }^{10}$ This corresponds to the default value of 1.5 for ACT-R's mismatch penalty scaling parameter.
} 
strategy of swapping arguments to make sure that the first is larger than the second (and therefore the extra rehearsals to facts of that type) is modeled by an additional probability of presentation of those problems that has also been estimated at $6 \%$.

\begin{tabular}{|c|ccccccccccccc|}
\hline & 0 & 1 & 2 & 3 & 4 & 5 & 6 & 7 & 8 & 9 & 10 & 11 & other \\
\hline $1+1$ & 0 & 0 & 97 & 2 & 0 & 0 & 0 & 0 & 0 & 0 & 0 & 0 & 0 \\
$1+2$ & 0 & 0 & 30 & 62 & 7 & 0 & 0 & 0 & 0 & 0 & 0 & 0 & 0 \\
$1+3$ & 0 & 0 & 28 & 9 & 60 & 2 & 1 & 0 & 0 & 0 & 0 & 0 & 0 \\
$1+4$ & 0 & 0 & 25 & 9 & 6 & 57 & 1 & 0 & 0 & 0 & 0 & 0 & 1 \\
$1+5$ & 0 & 0 & 24 & 8 & 6 & 4 & 55 & 1 & 0 & 0 & 0 & 0 & 3 \\
\hline $2+1$ & 0 & 0 & 20 & 75 & 5 & 0 & 0 & 0 & 0 & 0 & 0 & 0 & 0 \\
$2+2$ & 0 & 0 & 0 & 16 & 83 & 1 & 0 & 0 & 0 & 0 & 0 & 0 & 0 \\
$2+3$ & 0 & 0 & 1 & 24 & 38 & 33 & 3 & 0 & 0 & 0 & 0 & 0 & 1 \\
$2+4$ & 0 & 0 & 1 & 25 & 29 & 15 & 27 & 1 & 0 & 0 & 0 & 0 & 3 \\
$2+5$ & 0 & 0 & 1 & 26 & 30 & 5 & 13 & 18 & 0 & 0 & 0 & 0 & 7 \\
\hline $3+1$ & 0 & 0 & 15 & 10 & 73 & 2 & 0 & 0 & 0 & 0 & 0 & 0 & 0 \\
$3+2$ & 0 & 0 & 1 & 17 & 35 & 44 & 3 & 0 & 0 & 0 & 0 & 0 & 1 \\
$3+3$ & 0 & 0 & 0 & 0 & 21 & 9 & 70 & 1 & 0 & 0 & 0 & 0 & 0 \\
$3+4$ & 0 & 0 & 0 & 3 & 24 & 20 & 21 & 26 & 1 & 0 & 0 & 0 & 5 \\
$3+5$ & 0 & 0 & 1 & 3 & 26 & 11 & 27 & 4 & 14 & 0 & 0 & 0 & 14 \\
\hline $4+1$ & 0 & 0 & 14 & 9 & 7 & 69 & 1 & 0 & 0 & 0 & 0 & 0 & 1 \\
$4+2$ & 0 & 0 & 1 & 18 & 21 & 22 & 37 & 1 & 0 & 0 & 0 & 0 & 2 \\
$4+3$ & 0 & 0 & 1 & 2 & 19 & 22 & 18 & 34 & 1 & 0 & 0 & 0 & 4 \\
$4+4$ & 0 & 0 & 0 & 0 & 0 & 31 & 11 & 8 & 47 & 0 & 0 & 0 & 1 \\
$4+5$ & 0 & 0 & 1 & 2 & 3 & 21 & 16 & 7 & 8 & 14 & 0 & 0 & 28 \\
\hline $5+1$ & 0 & 0 & 14 & 9 & 7 & 5 & 63 & 1 & 0 & 0 & 0 & 0 & 2 \\
$5+2$ & 0 & 0 & 1 & 19 & 22 & 6 & 19 & 27 & 1 & 0 & 0 & 0 & 5 \\
$5+3$ & 0 & 0 & 1 & 2 & 20 & 9 & 27 & 6 & 24 & 0 & 0 & 0 & 11 \\
$5+4$ & 0 & 0 & 1 & 2 & 2 & 18 & 18 & 8 & 8 & 20 & 0 & 0 & 24 \\
$5+5$ & 0 & 0 & 0 & 0 & 0 & 0 & 47 & 13 & 9 & 6 & 18 & 0 & 7 \\
\hline
\end{tabular}

Table 2.4: Retrieval Frequencies for 5x5 Addition in Model.

Another way to examine this data is to plot the probability of correct retrieval for each argument as is done in Figure 2.2. Both plots show a fairly close match, with the jump in percentage correct for problems involving 1 , and the greater slope of the addend curve resulting from the probability of swapping arguments to further favor smaller addends.

\footnotetext{
${ }^{11}$ Answers in the "other" category are assumed to be retrieval failures resulting in guessing outside the 0 -to-10 range.
} 
Addition Retrieval (Augend)

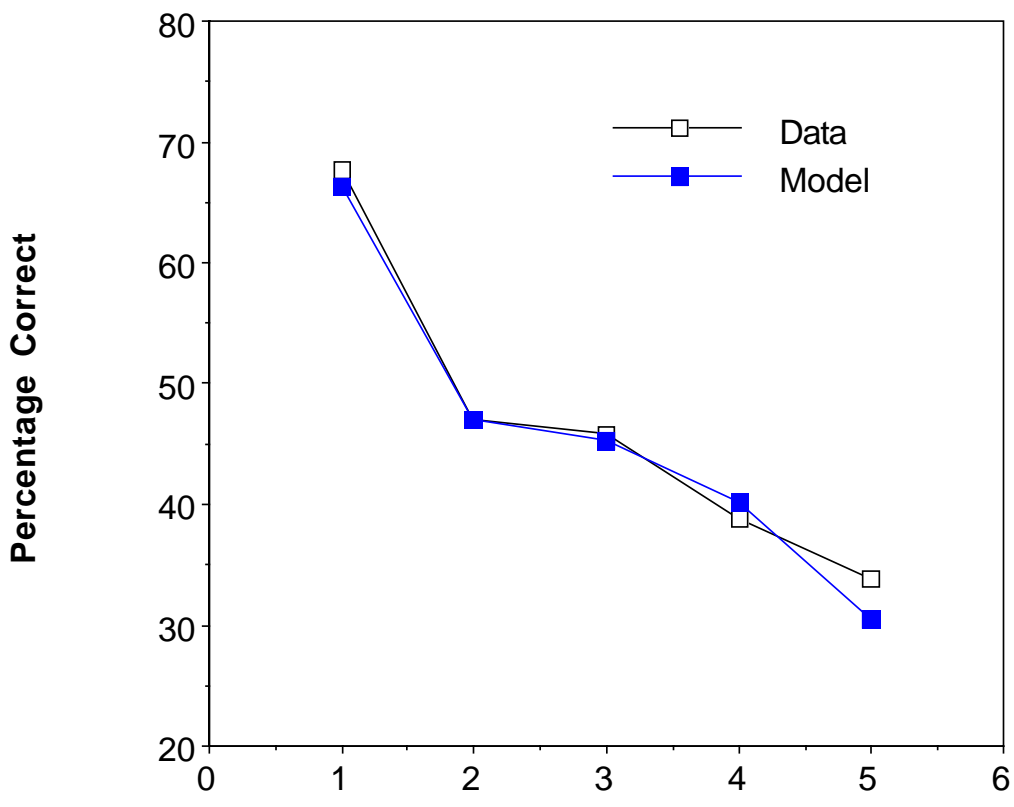

Augend

Addition Retrieval (Addend)

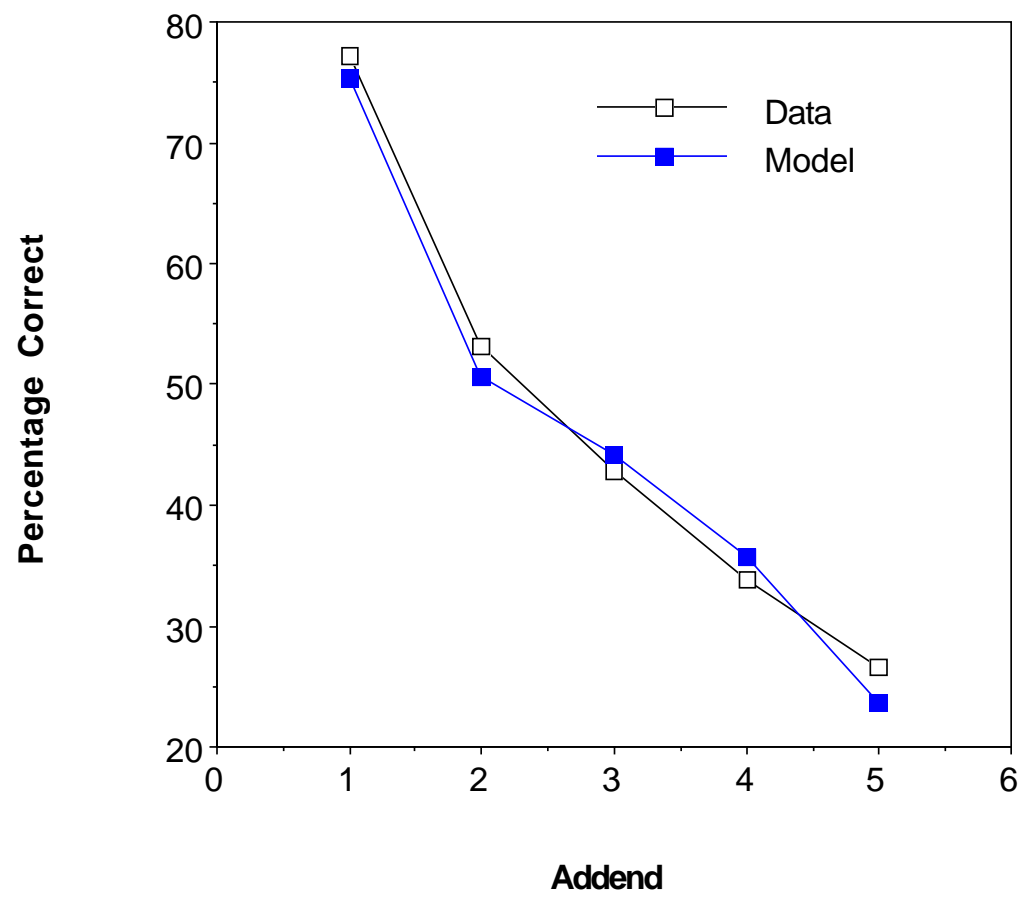

Figure 2.2: Percentage of Correct Retrievals in Addition: (a) Augend; (b) Addend. 
As was mentioned in the overview, this is a somewhat simplified model in that it assumes that only correct facts can be retrieved, albeit sometimes incorrectly. Of course, 4-yearolds may also hold some incorrect addition facts (at least weakly encoded), which if correctly retrieved will lead to error (or conversely if incorrectly retrieved could potentially yield a correct answer). In fact, the incorrect answers generated by the model's answer to this experiment would lead to just such incorrect facts. Another source of such errors could be results from past attempts at trying to reconstruct unavailable addition facts through counting (on their fingers or mentally) or other strategies. It is however not necessary to specify such past history since the basic assumptions of partial matching and a difference in rehearsal frequency can lead to a satisfactory model.

\subsubsection{Errors in Multiplication Computation}

Figure 2.3 from Siegler (1988) presents the percentage of errors in multiplication by repeated addition, a standard backup computation, for fourth-graders. Subjects were given single-digit multiplication problems in the form of a column of numbers in which the multiplicand was repeated the number of times specified by the multiplier, e.g. 8x6 was presented as a column in which 8 was written 6 times. Subjects were asked to add the columns of numbers and write down the answer. Analogous to the addition problems, the probability of error increases with the size of both the multiplicand and the multiplier. Particularly remarkable is the very low percentage of errors for repeated addition of 5.

Since multiplication by repeated addition essentially involves the same retrieval of arithmetic facts (counting and addition), the same mechanism can also explain that pattern of errors. Error percentage increases with the size of the multiplier because of the increase in the opportunities for retrieval error, and with the size of the multiplicand because of the increased probability of error in the retrieval of larger facts. The particularly low percentage of errors for repeated addition by 5 is obtained since only two facts are needed $(0+5=5$ and $5+5=10)$ and repeatedly reinforced, unlike other repeated additions where 5 or all 10 of the facts on that row of the addition table are needed. 


\section{Multiplication Computation (Multiplicand)}

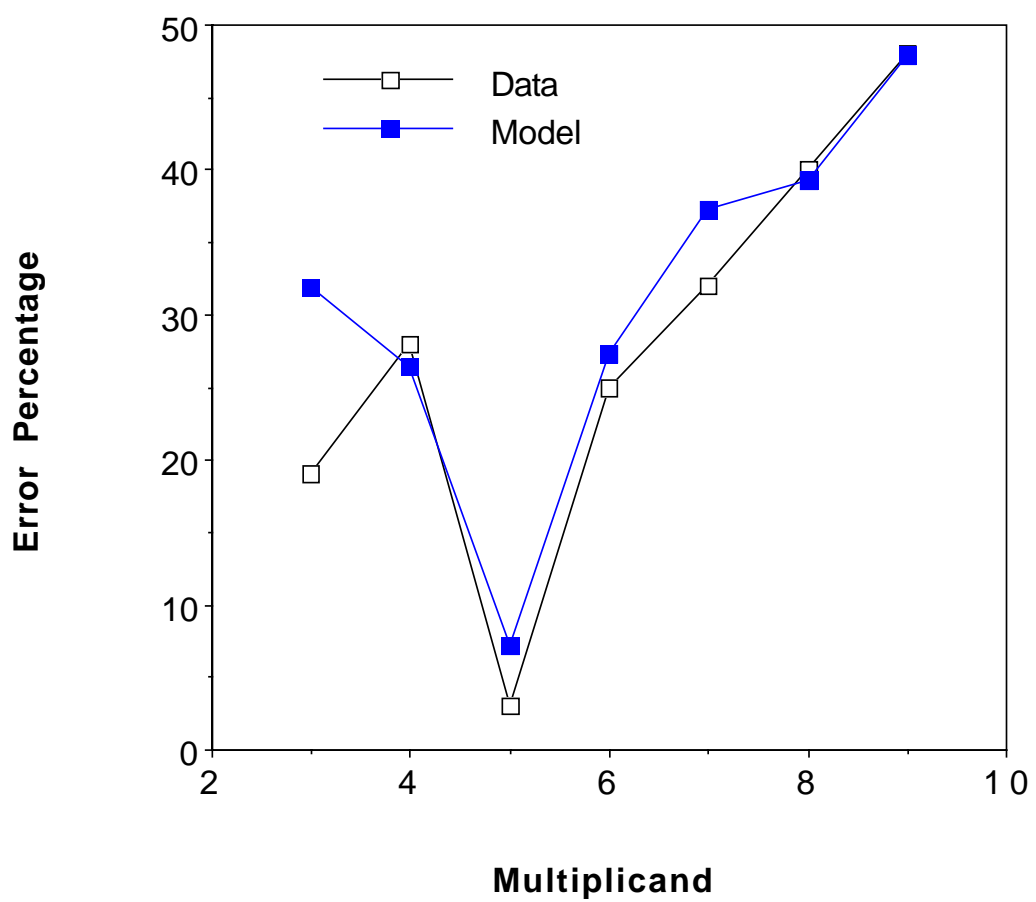

\section{Multiplication Computation (Multiplier)}

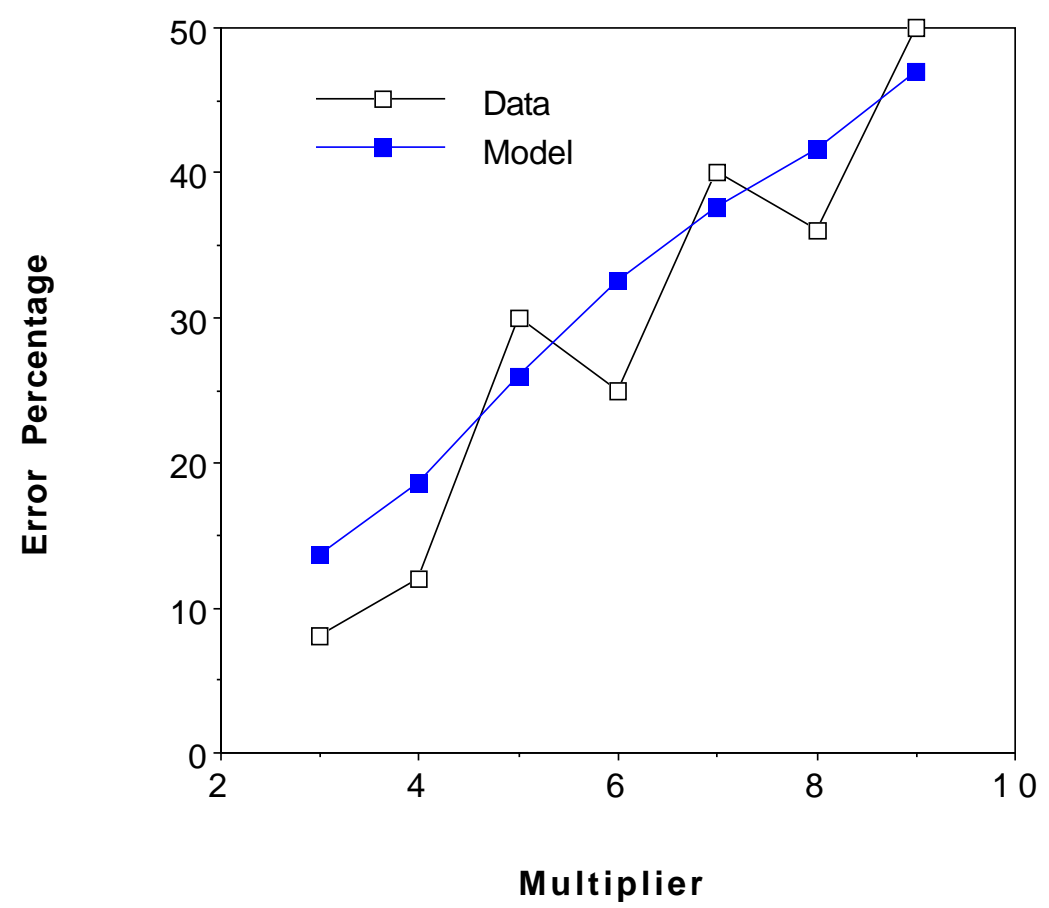

Figure 2.3: Percentage of Errors in Multiplication by Repeated Addition. (a) Multiplicand; (b) Multiplier. 
Figure 2.3 compares the predictions of the model with the data. Since the subjects were fourth-graders, this model assumes about four years of presentation of addition facts at a rate of a hundred a day, for a total of about one hundred and fifty thousand. The frequency ratio of this distribution is 4 . In addition, it is assumed that subjects have previously solved a certain number of multiplication problems by repeated addition. The distribution of multiplication problems is the one reported by Siegler for second- and third-grade textbooks. About a thousand multiplication problems are used resulting in five thousand additional addition rehearsals. The activation noise parameter $s$ is 0.12 and the same standard mismatch penalty factor of 0.15 per digit as used in the addition retrieval model. The plot by multiplicand shows a general increase in error percentage with the size of the argument, resulting from the decrease in rehearsal frequency for larger problems, and very few errors for addition by 5 , resulting from the limited set of facts needed (and, incidentally, the fact that they both contain the number 5 twice and therefore receive additional activation). The plot by multiplier also shows an increase in error for larger arguments, this time because the number of steps is directly proportional to the multiplier and each step introduces a new opportunity for error. One feature of the data that is not replicated by this model is the lower percentage of errors for even multiplier values. One possibility is that this may result from a hidden strategy of adding in pairs, e.g. adding 14 three times rather than adding 7 six times. 


\section{Chapter 3: Learning over Time}

The previous chapter described a number of cognitive arithmetic performance results at a particular point in the learning cycle and how to model them assuming a specific state of knowledge at that time. This chapter attempts to examine how these skills improve with time and how ACT-R's learning mechanisms can account for that.

\subsection{Problem Size Effect over Time}

Ashcraft (1987) describes the decrease in response time to addition problems across grades, as well as the gradual flattening of the problem size effect, from about a 2.5-to-1 ratio for large vs. small problems (two-digit sum vs. single-digit sum) in first grade to about a 1.1-to-1 by college. Figure 3.1a presents his data as a function of problem size and academic level of his subjects.

While some of this effect may be due to the gradual adoption over time of more efficient strategies (e.g. simply retrieving the fact instead of counting on one's fingers), the simplest way to account for it is by examining the increase in activation with practice and the resulting decrease in retrieval latency. Assuming that the frequency of presentation of each problem remains constant, the $S_{j i}$ values in the Activation Equation will also remain fairly constant and most of the effect of practice on activation will be reflected in the base levels of the facts. Thus, the critical equation is the Base-Level Learning Equation. If the number of references $n$ in the Optimized Learning Equation is replaced by $p . L$, where $p$ is the presentation rate in terms of number of presentations per unit of time and $L$ is the life of the chunk, then the Base-Level Learning Equation can be approximated by:

$$
B_{i}=\ln \frac{p \cdot L^{1-d}}{1-d}
$$




\section{Problem Size Effect (Data)}

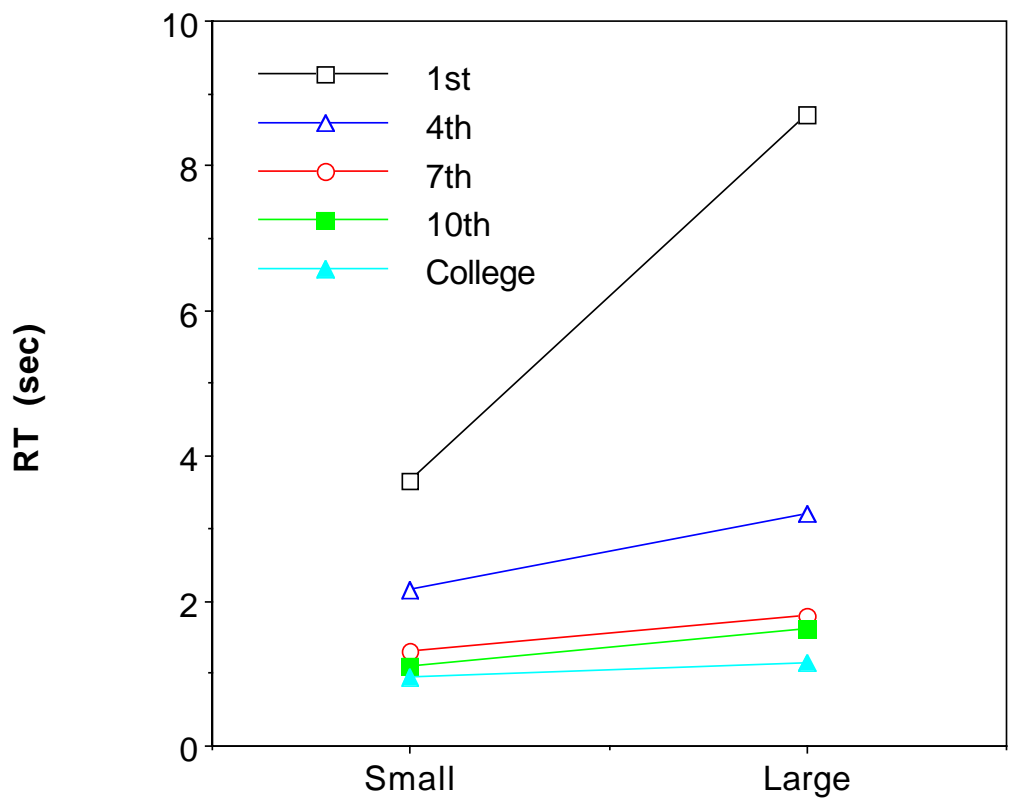

Problem Size

\section{Problem Size Effect (Model)}

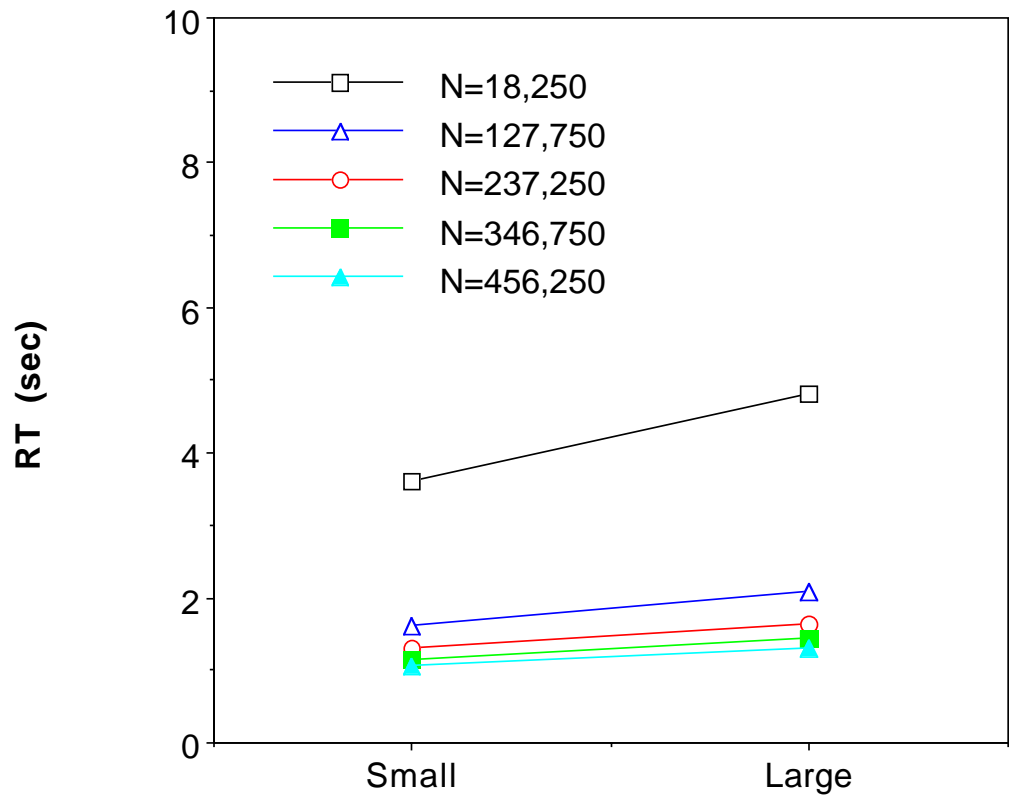

Problem Size

Figure 3.1: The Effect of Problem Size across Grades: (a) Data; (b) Model. 
Then by substituting this quantity into the Retrieval Time Equation, the retrieval latency can be shown to be a power function of the life of the chunk:

\section{Time $=c L^{-f(1-d)}$}

where $c=F p /(1-d)$ and so reflects the presentation rate $p$. Thus, the time to answer these addition problems is expected to speed up as a power function of age $(L)$. Figure 3.2 plots the data as a pair of small- and large-problem curves across grades with a log-log scale. It does appear roughly to speed up as a power law function of grade.

Power Law Speedup Over Time

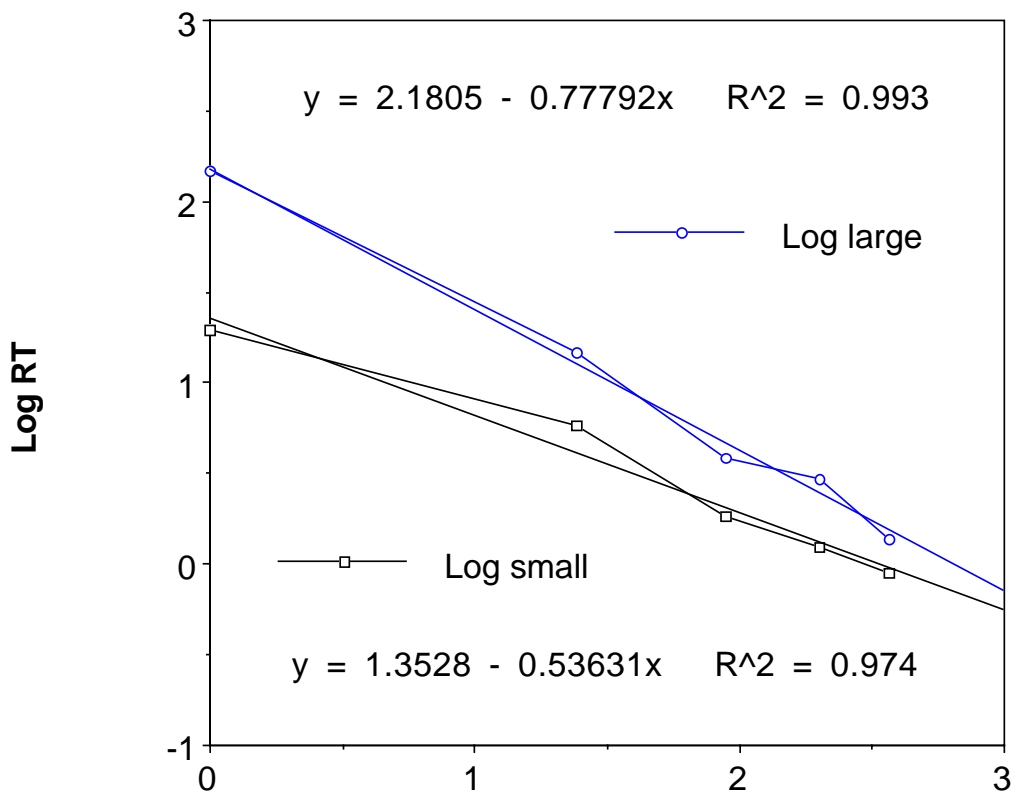

Log grade

Figure 3.2: Power-law Speed-up of Response Time

The slope of the small-problem curve of about 0.5 is perfectly compatible with the default parameter values of 0.5 for the base-level decay $d$. The somewhat higher slope of about 0.75 for the larger problems can be explained by a switch from computation to retrieval in addition to retrieval speedup, since first graders are still likely to use 
computation for some large problems. Another factor could be a leveling of the problem distribution over time, with large problems becoming gradually more frequent relative to small problems.

The results from the ACT-R model are displayed in Figure 3.1b. All parameters were the same as those used for the problem-size effect model previously presented. The amount of presentations for each grade corresponds to that many years of training (minus a half, assuming that subjects were tested in mid-year) at the usual average rate of a hundred problems a day. This model fails to produce the degree of speed-up for the large problems. The most probable explanation is its failure to include computation. Children are probably using this backup computation extensively for the large problems and it is producing a considerable slow down. ${ }^{12}$ However, Figure $3.1 \mathrm{~b}$ shows how much of the effect can be accounted for purely in terms of speed up in retrieval.

\subsection{Learning the Correct Answer}

\subsubsection{Overview}

Cognitive arithmetic performance increases over the years from marginal (less than 50\% correct retrieval of small addition facts among 4-year-olds as reported by Siegler and Shrager (1984), and even much worse for larger ones) to almost perfect and efficient retrieval for most adults under normal circumstances. At some point, children largely stop using the computation to answer their arithmetic problems and just retrieve the answer. They still make errors and according to the model there are two sources for errors, called type-a and type-b, on a problem like $3+5=$ ?:

(a) They will have stored incorrect answers (e.g., $3+5=7$ ) from past miscomputations and these can be retrieved.

\footnotetext{
${ }^{12}$ Chapter 4 on the lifetime simulation will show that this discrepancy goes away when a computation component is included.
} 
(b) They can partially match and retrieve a correct answer (e.g., 3+4=7) to a different problem.

What happens when a child starts retrieving answers subject to these errors and stops getting regular feedback on their additions? Can these errors be reduced through sheer practice at retrieval? This question will be answered separately with respect to these two types of errors in ACT-R.

First, will continued practice lead to a reduction in type-a errors? Every time the child retrieves the right answer or the wrong answer they will increase its base-level activation. Suppose $p_{1}$ is the frequency with which the correct answer is retrieved and $p_{2}$ is the frequency with which the incorrect answer is retrieved. Then from the earlier equation it follows that the difference in their base-level activations will be:

$$
B_{1}-B_{2}=\ln \frac{p_{1}}{p_{2}}
$$

which is a function of their relative frequencies $p_{1}$ and $p_{2}$. Thus, the difference in baselevel activations between a correct and incorrect fact will increase if and only if the difference in their frequencies increases. The associative activation will not change since, according to the Posterior Strength Equation, the $S_{j i}$ values only depend on the relative frequencies, not the amount of practice. Similarly, the other activation quantities (mismatch penalty, noise) do not change with practice. Under certain circumstances, the presentation frequencies and the base-level activations will diverge. This is essentially a rich-get-richer dynamics. Strong chunks (hopefully the correct ones) are more likely to be recalled, which will strengthen them further, while weak chunks (hopefully the wrong ones) will be increasingly less likely to be retrieved until they are gradually forgotten. The following sections present a mathematical analysis of this situation. It turns out that the critical parameter in this is ACT-R's noise parameter, $s$. If the parameter $s$ is set well below 1, ACT-R can "clean itself up" so to speak. Through repeated retrieval it will 
come more and more to retrieve the stronger answer and so strengthen its base-level activation.

The analysis of type-b errors is different. Under the assumption that the perfectly matching correct fact $(3+5=8)$ and the partially matching correct fact $(3+4=7)$ reflect problems which occur with a constant rate of frequencies, there will be no effect of practice on their relative base levels. Equally, mismatch penalty and noise will not change with practice. On the other hand, the critical factor concerns the associative strengths $S_{j i}$, between the cue 5 and the two facts $3+5=8$ and $3+4=7$. Again, under the assumption of not too much noise in the system, 5 becomes an increasingly good predictor of the perfect matching fact and an increasingly bad predictor of the partial matching fact. Since association strength reflects $\log$ odds and since 5 is associated with multiple facts, there is a bound on how strong the association between 5 and $3+5=8$ can be. However, there is no bound on how negative the association between 5 and $3+4$ $=7$ can become. As the odds goes to zero the associations can become unboundedly negative and so completely inhibit the mismatching fact. As future sections will establish, this requires that the value of the noise parameter $s$ be less than 1/3.

Figure 3.3 illustrates some results from a simulation in which the system starts out making a fair number of errors and eventually cleans itself up. The odds of commission errors decrease approximately as a power function of practice for a range of low noise values. The odds start at about 2.0 for the first block independently of the noise, but decrease by the hundredth block to about 0.02 for a noise variance of 0.1 and to 0.2 for a variance of 0.4 . The decrease is roughly linear on a log-log scale, confirming the powerlaw nature of the process. 


\section{Retrieval of $10 \times 10$ Addition Table}

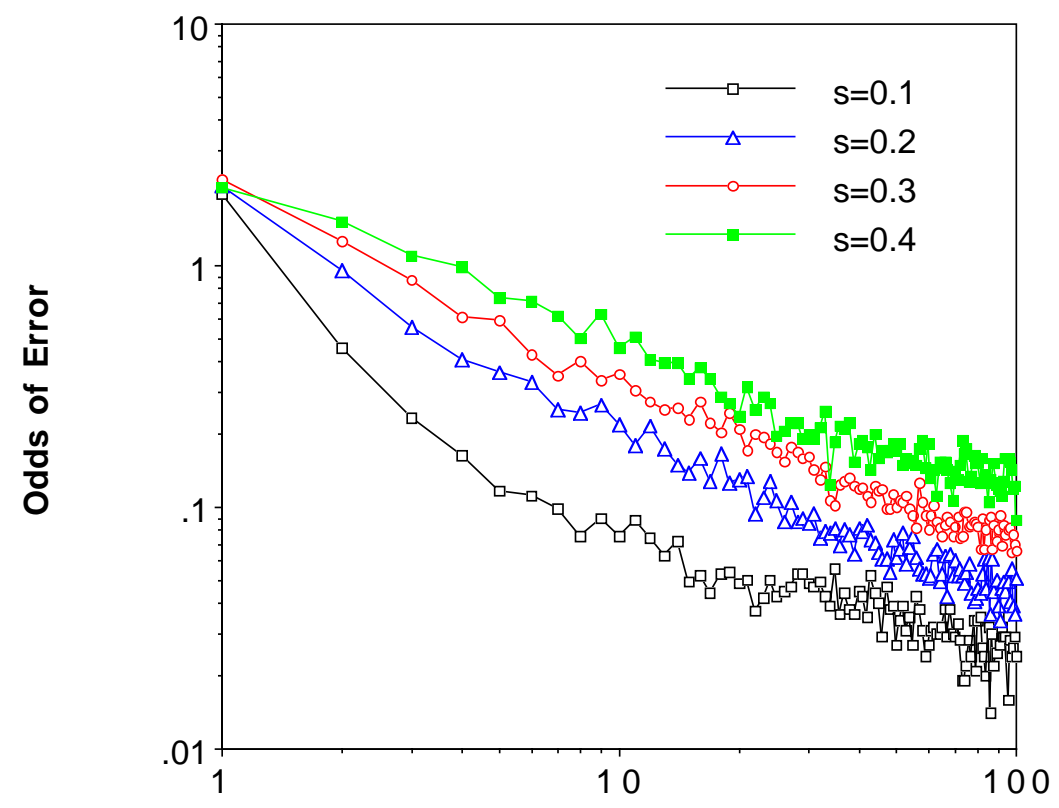

Block of 100 Problems

Figure 3.3: Power-law Decrease of Retrieval Errors (Model).

ACT-R's behavior with s values as in Figure 3.3 can be seen as the middle ground between two extreme strategies to deal with conflicting information, a.k.a. nonmonotonic knowledge (Bobrow, 1980). One would be to consider all facts to be immutable (as is the case for arithmetic knowledge) and reject any information that conflict with accepted knowledge. While this may be the right thing to do in the case of cognitive arithmetic, in general it leaves one overly determined by one's initial knowledge state and incapable of dealing with a changing, evolving world. The opposite strategy is to immediately reject previous knowledge when faced with conflicting information. While this may again be the right thing to do in situations where information is absolutely reliable, it could lead to catastrophic imbalance in many cases, including cognitive arithmetic. Consider, for instance, the consequences of trying to instantly rearrange your knowledge base if someone would tell you that $2+2=5$. Gradually shifting the strength of each piece of knowledge to reflect its strength of evidence (practice) is ACT-R's way of gracefully coping with conflicting knowledge. 


\subsubsection{Basic Dynamics of Error Odds}

To formalize the dynamics of retrieval, let us first assume that the two chunks $C_{1}$ and $C_{2}$ are competing for retrieval without any context. The Chunk Choice Equation can be rewritten to express the odds of chunk $C_{I}$ being retrieved as a function of the two chunks' activations and the activation noise level:

$O d d s_{1}=e^{\left(A_{1}-A_{2}\right) / s}$

Ignoring the contribution of associative activation and considering only base-level activation, the difference between the activations of $C_{1}$ and $C_{2}$ can be expressed using the equation giving the difference in base-level activation as the logarithm of the ratio of their presentation frequencies. This yields:

$O d d s_{1}=\operatorname{Ratio}_{1}^{1 / s}$

\section{Dynamic Odds Equation}

where Ratio $_{1}=p_{I} / p_{2}$ is the past ratio of the frequencies of retrieving $C_{1}$ and $C_{2}$. This equation shows that the current odds of retrieval are sensitive to the activation noise level. If $s>1$, the current odds of retrieval are closer to even odds than past history. This will ultimately lead to each chunk becoming equally likely to be retrieved. If $s=1$, the current odds of retrieval are equal to the past odds of retrieval. This does not imply that the retrieval odds will be fixed, but rather that they will drift randomly with experience, driven by chance and external events. If $s<1$, then the odds of retrieval become more extreme, with one becoming arbitrarily large and the other becoming infinitesimal. This is the winner-take-all dynamics mentioned previously. The noise level thus behaves much like the temperature in a Boltzmann machine: if it is too high, then the system is very disorganized and its entropy is maximized. If the noise is low enough however, the system gradually cools off until entropy is minimized and the system settles down to a fixed answer pattern. However, this analogy breaks down on the fundamental point of dynamics. In the Boltzmann machine, it is the explicit lowering of the temperature, 
which cools down the system and minimizes entropy. In ACT-R, the learning dynamics are responsible for the evolution of the system.

Each new experience will be added by the declarative learning mechanisms to the statistics of past history. This incremental change in the history of retrieval odds can be expressed by a differential equation, which for the $s<1$ case admits of two approximate solutions ${ }^{13}$ :

$$
\text { Ratio }_{1} \approx(c n)^{ \pm 1}
$$

\section{Rehearsal Ratio Equation}

which means that the past frequency ratio of retrieving either chunk gradually diverge according to a power law in the amount of practice of exponent -1 for the loser and +1 for the winner ( $c$ is a constant which depends upon initial conditions and $n$ is the total amount of practice). Combining this with the Dynamic Odds Equation, the current or observed odds of retrieving either chunk, and therefore the odds of commission errors, are a function of the amount of practice to the power of the inverse of the noise measure:

$$
O d d s_{1} \approx(c n)^{ \pm 1 / s}
$$

\section{Retrieval Odds Equation}

The Retrieval Odds Equation implies that the noise will determine the speed of convergence. But whereas a lower noise level implies a faster emergence of the winning, though not necessarily correct, answer, a higher noise level (still smaller than 1) causes slower convergence but a higher probability of the right answer emerging as the winner because the slower convergence lowers the impact of initial randomness.

Another way to view the Retrieval Odds Equation is in terms of the number of training examples needed to reach a particular accuracy. The number $n$ of presentations of a particular problem needed to lower the odds of confusion errors below some threshold $\varepsilon$

\footnotetext{
${ }^{13}$ Details of the derivation of the Rehearsal Ratio Equation can be found in the appendix.
} 


$$
n=\frac{1}{c \varepsilon^{s}}
$$

As a final comment, the power law form of the Rehearsal Ratio and Retrieval Odds Equations (or the sigmoid form of the equivalent probabilities) can also be found in the evolution of biological and technological systems between states of equilibrium (e.g. West \& Salk, 1987). This is probably related to the fact that these systems follow power law distributions similar to those of the cognitive environment (Anderson \& Schooler, 1991).

\subsubsection{Context and Complexity}

The previous section analyzed what is described previously as type-a errors, i.e. the competition between correct and incorrect answers through base-level strength. A similar analysis can be applied to type-b errors, the competition between two correct answers for different problems. Each will continue to be rehearsed because they are correct answers, but they will gradually become more sensitive to the exact features of the problem through the $S_{j i}$ values, which control spreading activation. Based on the discussion of the Posterior Strength Equation, the difference between the $S_{j i}$ values from the context $C$ to chunks $N_{l}$ and $N_{2}$ respectively is:

$$
S_{C N_{1}}-S_{C N_{2}}=\ln \frac{F\left(N_{1} \& C\right)}{F\left(N_{2} \& C\right)}-\ln \frac{F\left(N_{1}\right)}{F\left(N_{2}\right)}
$$

Assuming a total source activation level $W$ of 1 (the ACT-R default), then when adding base-level strength to spreading activation, the base level difference will cancel the second term of the previous equation and the difference in total activation between the chunks $N_{1}$ and $N_{2}$ is: 


$$
\Delta A=A_{1}-A_{2}=\ln \frac{F\left(N_{1} \& C\right)}{F\left(N_{2} \& C\right)}
$$

which means that the results derived in the previous section still hold, i.e. that the odds of retrieving either chunk in a given context is the same function of the past odds to the power of $1 / \mathrm{s}$ that was obtained in the context-free condition, but this time specific to the context.

But usually the context is not composed of a single chunk, and only part of the context can be used to differentiate between competing chunks. For example, if the problem is $3+4=$ ?, 4 is the only part of the context which can differentiate between $3+4=7$ and $3+5=8$. Since $\mathrm{W}$ must be divided among all three features (goals slots holding $3,+$ and 4 ) the 4 will only receive a $1 / 3$ weighting. Thus, the difference in activation between those chunks is:

$$
\Delta A=\frac{1}{3} \ln \frac{F(" 3+4=7 " \& 4)}{F(" 3+5=8 " \& 4)}
$$

It can be shown that the noise level $\mathrm{s}$ is divided by the $1 / 3$ factor and the odds equation becomes:

$O d d s=\operatorname{Ratio}^{1 / 3 s}$

This implies that the more complex the problem, i.e. the more sources of activation in the context, the lower the noise level needs to be to guarantee convergence.

As noted previously, this analysis assumes that $\mathrm{W}$, the total amount source activation, is equal to its default value of 1 . Anderson, Reder \& Lebiere (1996) have suggested that this assumption represents a fundamental limit of human cognition. Lovett, Reder \& Lebiere (1997; in preparation) have proposed that variations in $W$ can account for 
individual differences in processing capacity. If the value of $W$ is different from 1 , then the factor of $1 / 3$ used above generalizes to $W / 3$, and the previous equation becomes:

Odds $=$ Ratio $^{W / 3 s}$

As Anderson, Lebiere, Lovett \& Reder have noted, this implies that a larger $W$ effectively reduces the amount of noise and improves convergence, while a lower $W$ amplifies noise and limits the range of convergence. Thus the limit on $W$ is not only a limit on processing capacity but a limit on learning capacity as well.

\subsubsection{Matching Penalty}

This analysis focused on the influence of past rehearsal frequency through base level and spreading activation. An additional component of the Activation Equation is the mismatch penalty. The mismatch penalty $P$ biases the system in favor of one particular fact by adding or subtracting from the difference in activation between chunks:

\section{$\Delta A=\ln$ Ratio $\pm P$}

This introduces a factor proportional to the exponential of the penalty in the odds equation:

Odds $=\left(e^{ \pm P} \text { Ratio }\right)^{1 / s}$

While strongly biasing the initial odds toward the correct answer, the mismatch however does not directly affect the speed of convergence.

\subsubsection{Multiple Alternatives}

Until now the analysis of the odds of retrieval involved only two competing chunks. It 
can be shown from the Chunk Choice Equation that the odds of retrieving one of many alternatives is a direct function (the harmonic average) of the pairwise odds:

$$
\operatorname{Odds}(i)=\frac{1}{\sum_{j \neq i} 1 / \operatorname{Odds}\left(i_{\text {over }} j\right)}
$$

The same dynamic therefore applies in which the strongest alternative will get increasingly dominant over all others since it dominates each independently. This result is a variant of Luce's Choice Axiom (Luce, 1959).

\subsubsection{External Feedback Sources}

The analysis up to now assumed a closed system following its internal dynamics. Human cognition, of course, constantly interacts with the outside world. A particularly salient form of interaction in the case of cognitive arithmetic is teacher instruction. It can be shown that while teacher correction has a major impact on the Dynamic Odds Equation early on in the process, it becomes overwhelmed by the weight of experience if one allows the system to run uncorrected for a long time. This may be why ingrained errors are so hard to root out from human cognition.

Error correction will still be possible later on, but a much larger amount of correct feedback will then be necessary to reverse the odds in favor of the correct solution. This need to keep the system relatively stochastic early on in the learning to prevent the odds from growing large (and therefore less susceptible to correction) suggests a positive effect of activation noise upon long-term accuracy. By keeping the process sufficiently random early on, it prevents an occasional error (random or otherwise) from being locked in as the dominant answer too quickly and allows more time for the correct answer to emerge. Noise therefore performs a function similar to simulated annealing in a Boltzmann machine. In other words, noise is not (only) a shortcoming of the system but an essential contribution to its robustness in an imperfect environment. 


\subsubsection{Comparison}

A number of cognitive arithmetic models have been proposed (e.g. Ashcraft, 1987; Campbell, 1991; Siegler \& Shrager, 1984; and Siegler, 1988). While they differ in their focus, their details, and their complexity, they share a similar approach: they are based on the retrieval of facts from long-term memory, they employ a network-type approach where activation is spread and decays, and they control those processes using strengths which reflect past patterns of use.

ACT-R, an activation-based production system with Bayesian statistical learning of underlying real-valued parameters, is highly compatible with this approach. One of the details about which these models differ is the precise form of the decrease in error probability over time. Ashcraft (1987) increases the network strength values for correct associations (percentage of correct retrievals) yearly according to the formula:

\section{$\Delta$ strength $=g \cdot(100-$ strength $)$}

where $g$ is the growth rate estimated at 0.2. This equation originates from the incremental learning theory (e.g. Estes, 1964). It implies that the probability of error decreases exponentially with time:

$$
P(\text { error })=c e^{-g t}
$$

Siegler and Shrager (1984) and Siegler (1988) use a reinforcement rule that increments associations between problems and correct answers twice as much as associations between problems and erroneous answers. Although the exact form of the learning curve is not discussed, graphs in (Siegler and Shrager, 1984) suggest that the increase in the probability of a correct answer is roughly linear through most of the range until ceiling effects are encountered.

ACT-R's prediction of power-law decrease in retrieval error differs from those linear and 
exponential predictions. Error percentages in arithmetic retrieval from childhood to adulthood would enable us to choose among these theories, assuming that reliable data exist. As another data source, one could use error curves from artificial substitutes such as alpharithmetic.

Finally, the analysis presented here is entirely consistent with reports that convergence to perfect retrieval occurs at sharply different speeds among individuals and indeed may sometimes not happen at all. Goldman et al. (1988) conclude from data on the learning of addition facts by learning-disabled students that the performance of most of these children is developmentally delayed (rather than developmentally different) relative to that of normally achieving children. An obvious explanation to account for this result would be through the use of a larger noise value. The parameter analysis of Chapter 5 demonstrates that this is the case, but that other parameter variations can have the same effect as well. LeFevre et al. (1996b) also report that some undergraduate college students have not entirely switched to retrieval of multiplication facts and occasionally still use non-retrieval procedures. Again, this is consistent with this analysis, including a strong sensitivity of the convergence time to initial performance. 


\section{Chapter 4: The Lifetime Simulation}

The simulations presented in Chapter 2 all shared the same simplifying approach. To focus on a particular effect at hand, they assumed a certain distribution of knowledge strength at a particular point in time and proceeded to model the results given that state of knowledge and a particular set of parameter values. For example, the simulation of the problem-size effect assumed a distribution of strength for each arithmetic fact and derived the retrieval latency from these strengths. The simulation of the retrieval of addition facts by 4-year-olds assumed the distribution of strengths of those facts given the distribution of problems and backup procedures such as counting and derived the probability of correct retrieval and errors for each fact. The simulation of multiplication by repeated addition by 4th graders assumed a distribution of strengths for the addition facts used and derived the probabilities of errors for each problem. Even the evolution of the problem-size effect over time in the previous chapter relied on assumptions about the growth of strength of facts over time.

While this method is widely used in Cognitive Science and often produces both tractable analyses and excellent simulation fits, it suffers from a number of disadvantages. It requires additional assumptions about the state of knowledge at particular points in time. It allows different parameter values to be estimated for each fit. And it provides only an incomplete understanding of how each part fits with the others. For example, errors in the computation and retrieval of addition problems will lead to permanent erroneous facts, which in turn should impact the computation of multiplication by repeated addition. The only way to account for those interactions is to develop a single simulation to trace the evolution of knowledge and performance through the thousands of problems of the entire development cycle, from childhood to adulthood, all with the same set of parameters. That is the goal of the lifetime simulation described in this chapter.

Another benefit of the lifetime simulation is that it provides a stringent test of the 
architecture and in particular its learning mechanisms. Often an assumption or mechanism that seems to work well in short, static simulations will be revealed as inadequate in a long simulation with extensive learning. That is the case here. It should be noted that the learning taking place in this simulation (learning of new symbolic chunks and their sub-symbolic parameters: the base-level activations and associations strengths) is entirely declarative. The productions are assumed to have resulted from the appropriate instructional teaching, and their sub-symbolic parameters (strength, conflict resolution) are left at their default values throughout the simulation.

\subsection{Model}

The model of the lifetime simulation is essentially an assembly of the partial models described previously, with a few modifications. It models the impact of a lifetime of solving addition and multiplication problems. Table 4.1 displays the productions that are responsible for performing arithmetic by retrieval.

\section{Arithmetic-Retrieval}

IF the goal is to solve an arithmetic problem of the type X OP Y and there is a fact stating that $\mathrm{X} O \mathrm{OP} \mathrm{Y}=\mathrm{Z}$

THEN set the answer as $\mathrm{Z}$

\section{Done-Arithmetic}

IF the goal is to solve an arithmetic problem and the answer has been found THEN output the answer and pop the goal

\section{First-Plus-Zero}

IF the goal is to solve an arithmetic problem of the type $\mathrm{X}+0$

THEN set the answer as $\mathrm{X}$

\section{Zero-Plus-Second}

IF the goal is to solve an arithmetic problem of the type $0+\mathrm{X}$

THEN set the answer as $\mathrm{X}$

\section{Double-Recoding}

IF the goal is to solve an arithmetic problem of the type X OP X THEN recode the problem as X OP Double

Table 4.1: Basic Arithmetic Productions. 
The main production, Arithmetic-Retrieval, solves an arithmetic problem by retrieving the corresponding fact. The production Done-Arithmetic outputs the answer and pops the goal. Addition problems of type " $x+0$ " and " $0+x$ " are directly solved without retrievals by the special-purpose productions First-Plus-Zero and Zero-Plus-Second, respectively. The production Double-Recoding recodes tie problems of the form " $x+x$ " as " $\mathrm{x}+$ Double" where Double is a special chunk indicating a redundant argument. This representational change will be discussed in Section 4.3. Table 4.2 displays the productions that perform addition by backup computation i.e. repeated counting.

\section{Addition-Counting}

IF the goal is to solve an arithmetic problem of the type $\mathrm{X}+\mathrm{Y}$

THEN set a subgoal to count from $\mathrm{X}$ a number of times equal to $\mathrm{Y}$

\section{Done-Count}

IF the goal is to count $\mathrm{X}$ times and the counter is $\mathrm{X}$

THEN return the result and pop the goal

\section{Iterate-Count}

IF the goal is to count from $\mathrm{X}$ and the counter $\mathrm{Y}$ is less than the limit $\mathrm{Z}$

THEN set a subgoal to increment $X$ and a subgoal to increment $\mathrm{Y}$

\section{Count-Up}

IF the goal is to increment the number $\mathrm{X}$ and the number following $\mathrm{X}$ is $\mathrm{Y}$

THEN return the number $Y$

\section{Double-Counting}

IF the goal is to solve an arithmetic problem of the type $\mathrm{X}+$ Double THEN set a subgoal to count from $X$ a number of times equal to $X$

Table 4.2: Productions for Addition by Repeated Counting.

The production Addition-Counting generates a subgoal to iteratively count up to the answer from the first operand to the second. The production Iterate-Count counts up by setting subgoals to increment the answer and the counter. The production Count-Up does the actual counting by retrieving the counting facts and popping those subgoals. This practice of setting subgoals to perform retrievals will be discussed in Section 4.4. The production Done-Count recognizes when the counter has reached the limit of the 
second operand and pops the answer. The production Double-Counting is the equivalent of Addition-Counting for the tie problems in format " $\mathrm{x}+$ Double" resulting from DoubleRecoding. Similarly, Table 4.3 displays the productions that solve a multiplication problem by iteratively adding the multiplicand an amount of times equal to the multiplier.

\section{Multiplication-Adding \\ IF the goal is to solve an arithmetic problem of the type $\mathrm{X} * \mathrm{Y}$ \\ THEN set a subgoal to add Y X times \\ Iterate-Add \\ IF the goal is to add $\mathrm{X}$ times and the counter $\mathrm{Y}$ is less than $\mathrm{X}$ \\ THEN set subgoals to increment the counter, increment the units digit, split the result to extract the carry and increment the tens digit by the carry}

\section{Construct-Result}

IF the goal is to add $\mathrm{X}$ times and the counter is $\mathrm{X}$ THEN set a subgoal to merge the tens and units digits

\section{Done-Add}

IF the goal is to add $\mathrm{X}$ times and the counter is $\mathrm{X}$ and the tens and units digits have been merged THEN return the result and pop the goal

\section{Split}

IF the goal is to split the number $\mathrm{X}$

THEN return the tens and units digits of number $\mathrm{X}$

\section{Merge - Numbers}

IF the goal is to merge the tens digit $\mathrm{T}$ and units digit $\mathrm{U}$

THEN pop the goal

\section{Double-Adding}

IF the goal is to solve an arithmetic problem of the type $\mathrm{X} *$ Double THEN set a subgoal to add $\mathrm{X} X$ times

Table 4.3: Productions for Multiplication by Repeated Addition.

The production Multiplication-Adding sets a subgoal to solve a multiplication problem by repeated addition. The production Iterate-Add subgoals the operations to execute one step of repeated addition. When all the steps have been completed, the production Done-Add pops the answer. Since only single-digit addition facts are systematically 
stored, the Iterate-Add production performs multi-digit addition by splitting the numbers into tens and units digits using the Split production, incrementing tens and units digits separately, then finally reconstitutes the digits into a single number using the MergeNumbers production and returns the result as the answer. The production DoubleAdding is the equivalent of Multiplication-Adding for the tie problems in format " $\mathrm{x}$ *Double" resulting from Double-Recoding. These productions had the usual default parameters. Exceptions are the special-purpose First-Plus-Zero and Zero-Plus-Second productions, whose action latency was set at 0.7 second, and the Iterate-Count production, whose action latency was set at 0.5 second. The latter latency is to account for the context-switching part of the counting task, either in the form of subgoal creation and pushing, or external strategies such as finger-counting.

Finally, many of the parameters for the lifetime simulation were left to their default values. The base-level decay rate was set to 0.5 as usual. The weight of the prior in the Posterior Strength Equation was set to 1.0 by default, i.e. the prior values set at creation carry a weight equal to any retrieval. As argued in Whalen (1996), a similarity measure that was sensitive to the magnitude of numbers was introduced. Perhaps the simplest such measure was used, in which the similarity between numbers is equal to the ratio of the smaller number to the larger number. This similarity measure has the advantage of scalability, i.e. the similarity between 3 and 5 is the same as the similarity between 30 and 50, an intuitively desirable property. The mismatch penalty was left at its default value of 1.5 . The activation noise $s$ was set to 0.25 , which has become a fairly standard value in other ACT-R models (Lebiere \& Wallach (in preparation), West \& Lebiere (in preparation)). The retrieval threshold was set to -3.75 and the latency factor to $0.125^{14}$. Both values are fairly low by ACT-R standards (e.g. Anderson, Bothell, Lebiere, \& Matessa (1998)), but this is an unusually long simulation, which means that unlike laboratory experiments an enormous amount of cognition happens between problem presentations. This would be reflected among other places in a much larger $\mathrm{F}$ factor in

\footnotetext{
${ }^{14}$ This yields retrieval latencies as high as 5 seconds. While this seems unusually high compared to the typical sub-second retrievals reported in laboratory experiments, young children often do take that long to retrieve an answer.
} 
the Posterior Strength Equation that counts the total number of production firing, leading to higher associative activation. Another parameter that could be used to estimate the impact of the rest of one's cognitive life upon one's arithmetic performance would be the base-level constant in the Base-Level Learning Equation. Adding either of both of these factors would allow for higher, more usual values of the retrieval threshold and latency factor, at the cost of an additional parameter. Chapter 5 will systematically analyze the impact of the activation noise, retrieval threshold and mismatch penalty upon the performance of the model.

Another set of parameters is specific to the lifetime simulation and determines the distribution of problems over time. In accordance with the studies of the textbook presentation frequencies of basic addition and multiplication facts in Hamman \& Ashcraft (1986) and Ashcraft \& Christy (1995), the students were exposed to an estimated 2000 addition problems per year and the same number of multiplication problems, except for the first grade when all the problems were addition problems. ${ }^{15}$ This corresponds in the lifetime simulation to an average inter-problem delay of about 7500 seconds, or about two hours. In accordance with the same studies, a ratio of about 2.6 to 1 between most frequent $(0+0)$ and least frequent $(9+9)$ problem is assumed. Finally, to simulate the amount of feedback received from a classroom environment, subjects were given the answer when they couldn't retrieve it with a probability initially equal to 1 that decreased over time according to their probability of retrieval. Chapter 5 also systematically examines the impact of those domain parameters on the lifetime simulation.

The lifetime simulation runs through 20 years of training, for a total of 40,000 addition problems, and almost as many multiplication problems. On a 300MHz G3 PowerMac, it takes about an hour and a half to run. The fact that this degree of compression can be achieved is testimony to the considerable efficiency of the ACT-R simulation language, even with all of its real-valued computations enabled. ${ }^{16}$ A human doing nothing else

\footnotetext{
${ }^{15}$ This total doesn't include the use of addition facts to solve multiplication problems.

${ }^{16}$ This is with the Optimized Learning (:ol) flag to use the Optimized Learning Equation.
} 
would take between one and two orders of magnitude longer to solve the same number of problems.

\subsection{Results}

The previous section described the structure of the lifetime simulation model, which is essentially the same as the previously introduced partial models, except for a few minor changes. While the models themselves fit together nicely, an important question was whether the separate models with their different parameter values used to model each separate result could be unified in a single lifetime simulation which could reproduce the entire set of results with the same parameters. The answer is affirmative, and this section will describe each result and how it was obtained. Unless otherwise indicated, these results represent the average of 20 runs of the lifetime simulation. Figure 4.1 presents the results of this simulation in terms of the problem size effect at various ages.

\section{Problem Size Effect over Time}

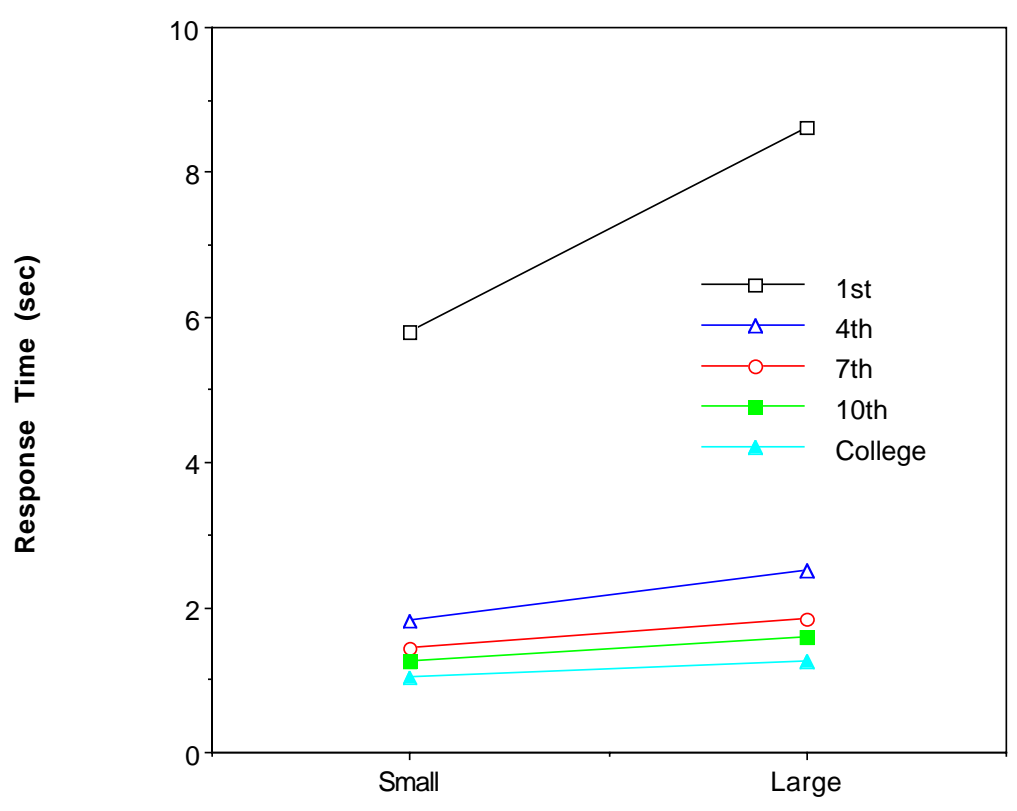

Problem Size

Figure 4.1: Problem Size Effect over Time for Small and Large Facts. 
The results of the lifetime simulation are even better than those of the previous simulation (Figure 3.1) which only took retrieval into account, because the additional latency of backup computation provides a closer fit to the long response times for large problems in the early grades. The response times are decreasing not only because the answers are getting stronger but also because the model is increasingly switching from computation to retrieval. The latency for small problems in the first grade is a little high, but that might reflect the training on those problems received during pre-school and kindergarten, which is not modeled by the lifetime simulation which starts with the first grade. Indeed, the data set for retrieval of small addition facts by 4-year-olds indicates a significant amount of knowledge long before the first grade.

Because the lifetime simulation models school learning from the first grade on, the data set for 4-year-olds was modeled with a separate run of the lifetime simulation with slightly different parameters to represent the different conditions. All the parameters were the same, except that the training set was restricted to problems up to $6+6$, to provide the same range of answers as the experiment and on the assumption that 4-yearolds did not have much exposure to double-digit facts. The initial probability of feedback during training was also lowered from 1.0 to 0.5 , leading to backup computation up to half the time. As in the previous simulation, a thousand training problems were presented, after which retrieval was tested. The probabilities of correct retrieval as a function of argument size are plotted in Figure 4.2. They closely fit the data (Figure 2.2), with a problem size effect for addend slightly larger than for augend. This reflects the past use of backup computation, since the effect of addend (i.e. number of iterations) is larger for computation than the effect of augend (i.e. larger numbers). Therefore, although the simulation is solving the problems by retrieval instead of counting (as were the subjects), the past computation errors are reflected in the retrieval performance. 


\section{Percentage Correct for Addition Retrieval in the First Cycle (1000 Problems)}

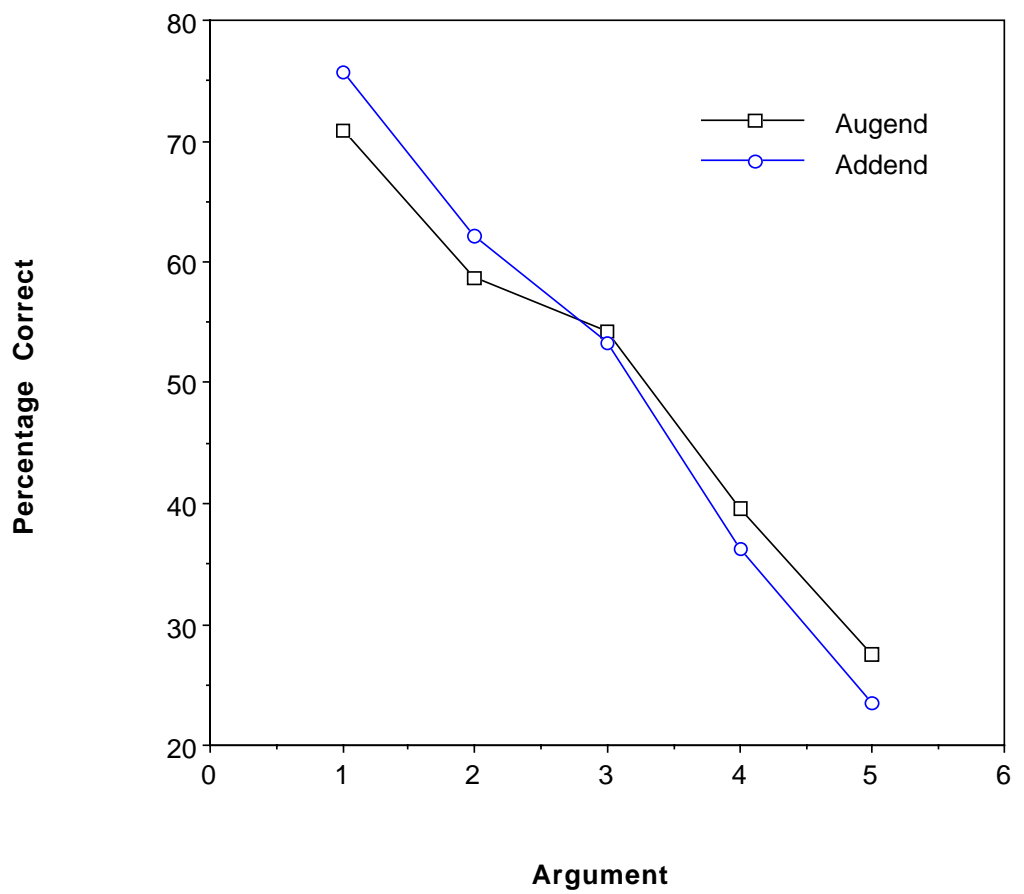

Figure 4.2: Percentage Correct in Retrieval of Small Addition Facts After Training on 1000 Problems.

The multiplication computation data is obtained from the performance of the lifetime simulation on multiplication by repeated addition during the third cycle, which corresponds to the fourth grade. Figure 4.3 plots the percentages of error as a function of the multiplicand and multiplier. The lifetime simulation reproduces the problem size effect for both multiplicand and multiplier (Figure 2.3). The much lower error rate for the multiplicand 5 is present, resulting from the lower error rate for the two main singledigit addition facts used in counting by 5 , i.e. $0+5=5$ which is solved by the zero rule, and $5+5=10$, which is a tie problem. The lifetime simulation produces an apparent evenmultiplicand effect, resulting from the fact that even multiplicands only use half of the facts in their row of the addition table, and thus are more reliable. Oddly, the data displays an apparent even-multiplier effect (Figure 2.3(b)). The overall error rate is slightly lower but comparable to that of the fourth graders. 
Error Percentage for Multiplication

Computation in Cycle 3 ( 4th Grade)

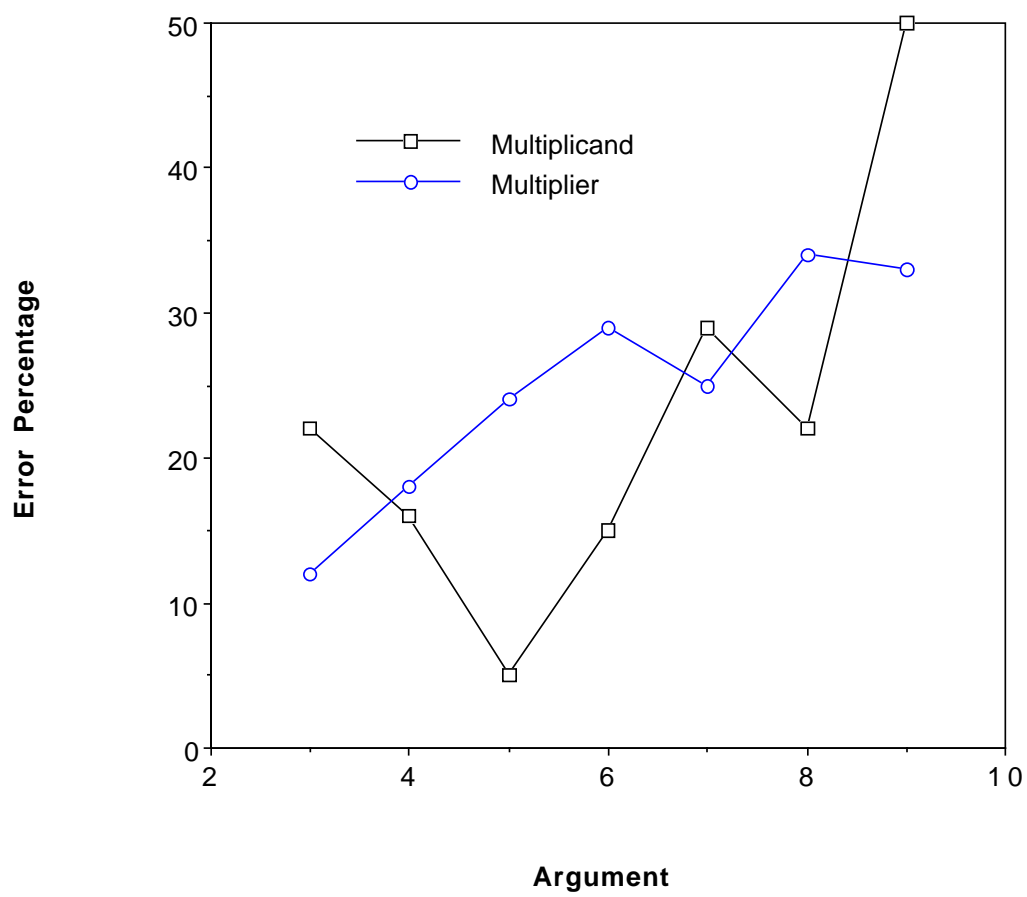

Figure 4.3: Error Percentage as a Function of Argument for Multiplication Computation in the $4^{\text {th }}$ Grade.

One can look at the detailed latency pattern for addition facts at the end of the simulation, which corresponds to young adults (Figure 2.1). Figure 4.4 plots the response time for zero, tie and other single-digit addition problems. Although an occasional computation is performed, the latencies here overwhelmingly reflect the retrieval latencies for the correct fact, or the constant time ( 0.7 second) of application of a rule for the problems involving zero, plus a constant time for encoding the problem and outputting the result $(0.2$ second). The curve for tie facts is lower and flatter than the curve for non-zero, non-tie facts. This is due not only to the increased spreading activation to those facts, but also to the fact that since those problems could be retrieved earlier and more reliably than the others, they have received a comparatively higher amount of reinforcement. 
Problem Size Effect for Zero, Tie and Other Problems

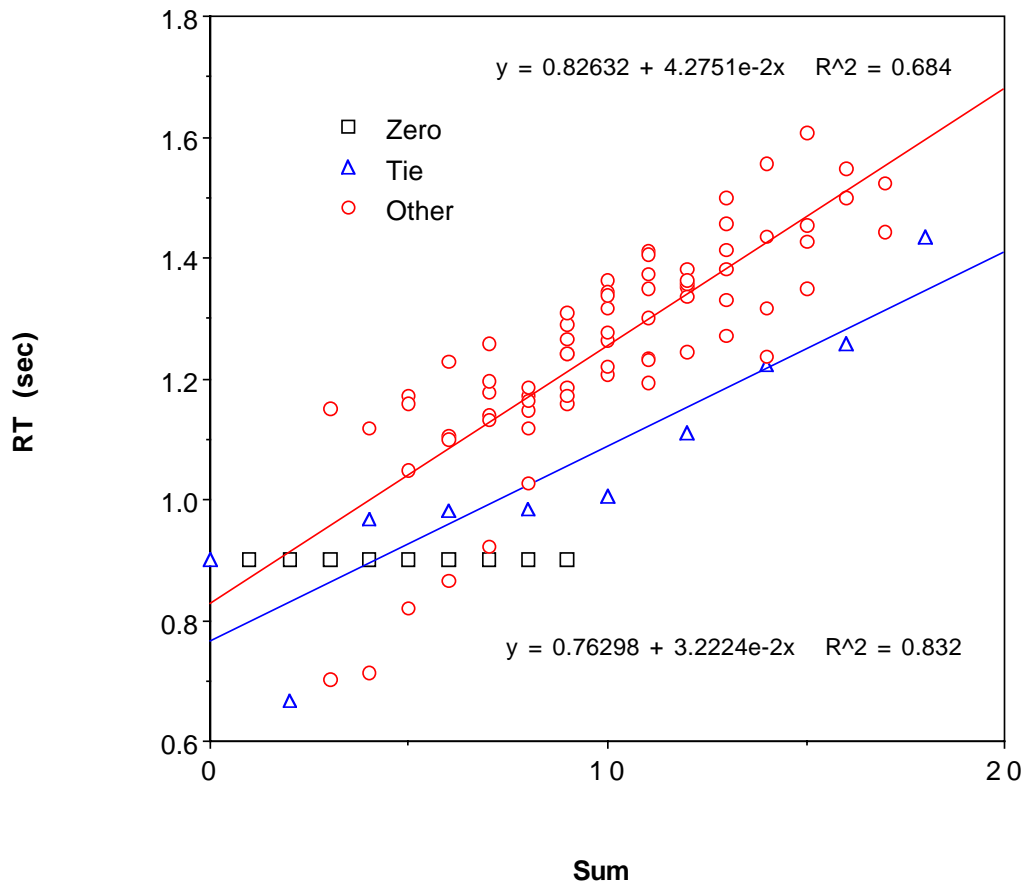

Figure 4.4: Problem Size Effect for Zero, Tie and Other Problems At the End of the Lifetime Simulation

Finally, one can look at the behavior of the lifetime simulation to confirm the formal analysis of Chapter 3 predicting a power-law decrease over time of the odds of retrieval failure (hence computation) and the odds of retrieval error. Figure 4.5 plots the odds of retrieval error and the odds of computation for addition problems as a function of practice for addition and multiplication problems. Both the odds of retrieval error and the odds of computation clearly decrease as a power law. The fit is even closer for multiplication problems, which might be due to the fact that while the learning of multiplication intruded upon the learning of the addition facts and made use of those facts, no higher level skill made use of multiplication facts, leaving their learning curve perfectly unaltered. 
Odds of Retrieval Error and Computation for Addition Problems as a Function of Practice

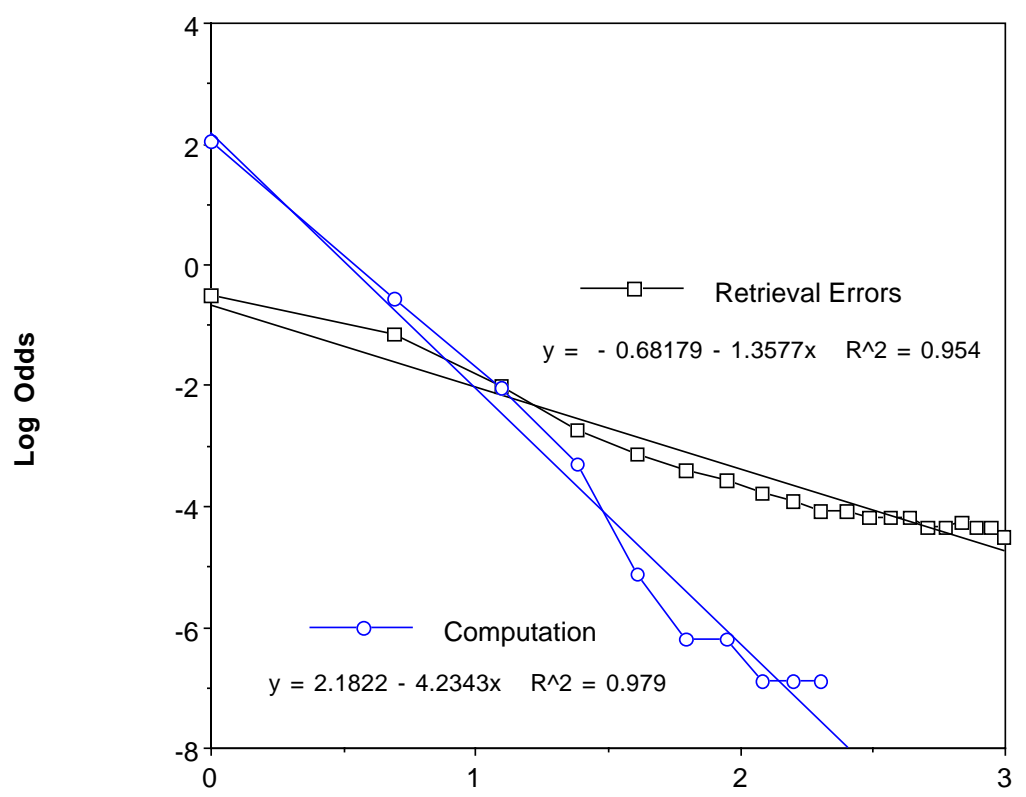

Log Cycle

Odds of Retrieval Error and Computation for Multiplication Problems as a Function of Practice

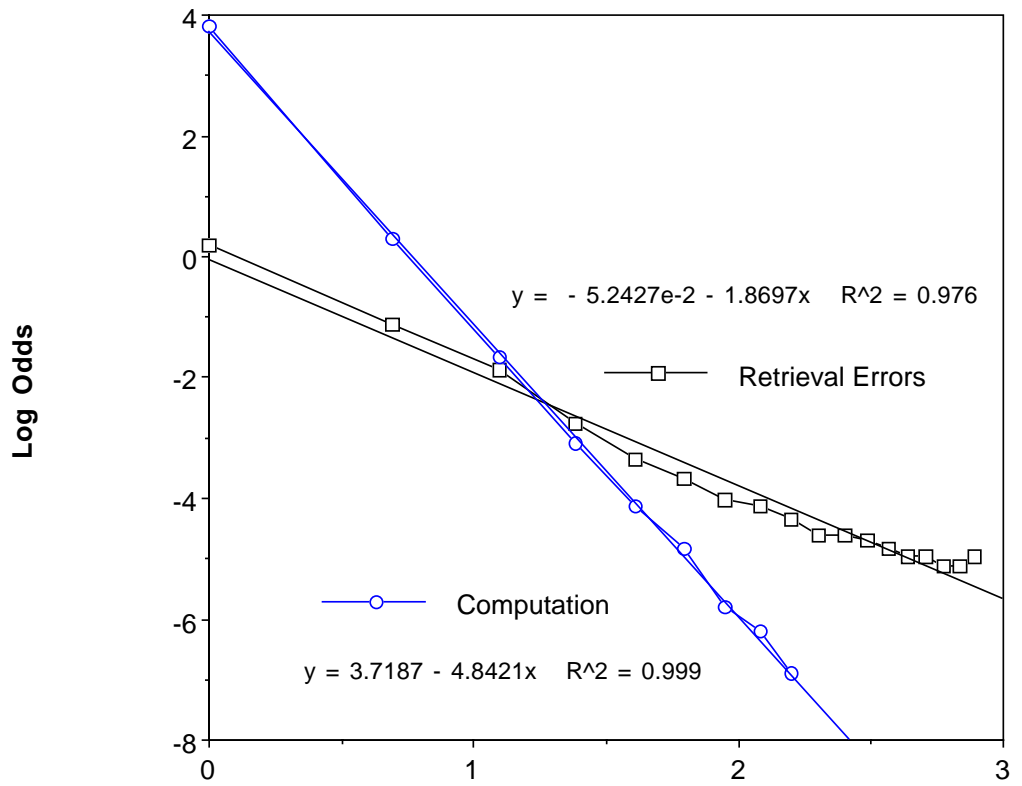

Log Cycle

Figure 4.5: Odds of Retrieval Error and of Computation as a Function of Practice: (a) Addition; (b) Multiplication 
In addition to the data fits previously reported, these computation and retrieval error curves generally fit the data reported by Siegler \& Robinson (1982), Siegler \& Shrager (1984) and Siegler (1988) on the percentage of strategy use and retrieval errors at various points of development. Together, these results, and a few more presented in later chapters, provide a strong corroboration of this model and ACT-R's theory of learning.

\subsection{Associative Strengths and Interference}

\subsubsection{General Introduction}

In the Posterior Strength Equation, the empirical value, weighted by the number of observations $F\left(C_{j}\right)$, will come to dominate the prior, weighted by the constant parameter $\operatorname{assoc}^{17}$, and the $R_{j i}$ ratio will converge to the empirical ratio $E_{j i}$ :

$$
E_{j i}=\frac{F\left(N_{i} \& C_{j}\right) / F\left(C_{j}\right)}{F\left(N_{i}\right) / F}=\frac{F\left(N_{i} \& C_{j}\right) \cdot F}{F\left(N_{i}\right) \cdot F\left(C_{j}\right)}
$$

where $F\left(N_{i} \& C_{j}\right)$ is the number of times that $i$ was needed when $j$ was in the context, $F\left(C_{j}\right)$ is the number of times that $j$ was in the context, $F\left(N_{i}\right)$ is the number of times that $i$ was needed and $F$ is the number of production firings since $i$ was created.

In this domain, $j$ is usually a number or arithmetic operator and $i$ is an arithmetic (or counting) fact. ${ }^{18}$ If the number $j$ is usually not in the context when $i$ is needed, then the empirical ratio will be very small since $F\left(N_{i} \& C_{j}\right)$ will be much smaller than $F\left(N_{i}\right)$, and the associative strength $S_{j i}$, which is equal to the logarithm of the ratio $R_{j i}$, will be very negative. This seems a bit surprising since the Prior Strength Equation specifies that

\footnotetext{
${ }^{17}$ The Associative Learning (:al) global parameter, set to 1.0.

${ }^{18}$ This pattern of associative strengths running from basic components of the domain to chunk representing small groups of these components is general enough to suggest a dichotomy in declarative memory between basic referable elements and higher-order
} 
unconnected chunks should have an initial strength of 0 . However, that is merely a convenient simplification. In the case of connected chunks (i.e. $j$ appears in a slot of $i$ ), the value of $F\left(N_{i} \mid C_{j}\right)$ is assumed to be $1 / n$, where $n$ is the number of chunks connected to $j$. That means that the total probability of retrieving a chunk connected to $j$ is 1 , which implies that the probability of retrieving a chunk unconnected to $j$ is 0 . Since the prior strength of association is the logarithm of the ratio of that probability over the base probability, if the Prior Strength Equation were consistent that would mean that the prior strength between unconnected chunks should be minus infinity instead of 0 . However, that would immediately preclude the retrieval of any chunk when an unconnected chunk is in the context, a somewhat extreme situation. However, given the proper training, the Posterior Strength Equation indeed specifies that those values will become increasingly and unboundedly negative.

If one makes the opposite assumption that the number $j$ is always in the context when $i$ is needed (e.g. 4 is always in the goal when $3+4=7$ is retrieved), then the empirical ratio will tend to $F / F\left(C_{j}\right)$, which will be stable over time if numbers are presented fairly uniformly. Interestingly, if one assumes that all chunks were created at about the same time (an assumption that is only approximately true for arithmetic chunks but that becomes increasingly true as time passes), then $F$, the number of production firings since the creation of the chunk, simplifies away and the strength of association from a chunk $j$ becomes inversely proportional to $F\left(C_{j}\right)$, its frequency of appearance in context. In this case, that means that larger numbers, which appear less often, will spread more activation to their connected chunks than smaller numbers do.

This will tend to mitigate the effect of frequencies upon base-level strength, but only partially. To see that, let us assume that the frequencies of facts in the addition table decreases linearly with each operand, and consider the extreme chunks in the table: $0+0$ and $9+9$. If $0+0$ is 3 times more frequent than $9+9$, then according to the approximate form of the Base-Level Learning Equation, the difference between the base-level activation of $0+0$ and $9+9$ is the logarithm of their frequency ratio, i.e. $\ln (3)$. However,

non-referable chunks. 
because 0 appears as a source for chunks that are less frequent than $0+0$ and 9 appears as a source for chunks that are more frequent than $9+9$ (e.g. $0+9$ features both and is twice as frequent as 9+9), then the average frequency of 0 being a source is only $2 / 3$ higher than the average frequency of 9 being a source. In other words, the frequency distribution of chunks is always more extreme than the aggregate distribution of their components. In this case, the difference between their sources of activation is only $\ln (1.6)$, which is significantly less than the difference in base-level activation. This effect of frequency upon strengths of association is also reduced because all addition chunks have one source in common, i.e. the operator + , leaving only $2 / 3$ of the total source level to be frequencysensitive. Of course, a value of $W$ higher than 1 will tend to amplify this effect of strengths of activation, but presumably not to the point where it would overwhelm the primary frequency effect on base-level activation.

This effect is somewhat surprising and is in fact a variant of the fan effect (e.g. Anderson $\&$ Reder, in press), that states that the activation spread from a source to related chunks will decrease with the number of related chunks. In this case, the activation spread decreases with the number of appearances of the source in the context, irrespectively of how many chunks it is connected to. In any case, it is a good thing that the effect of frequency on the base-level strength predominates since if the strengths of association did, then ACT-R would predict that chunks that are retrieved more often are actually less active and take longer to retrieve than chunks that are retrieved less often, a clearly undesirable result. Indeed, this inversion of the problem size effect sometimes appears for pathological parameter values, as will be demonstrated in Chapter 5 .

A more serious problem results from the fact that the Prior Strength and Posterior Strength Equations, which specify the default and learned values of the associative strengths respectively, take into account every appearance of a chunk in the context, independently of the type of the retrieved chunk or the slot in which it appears. This assumption can lead to some counterintuitive results involving interference among facts: between operands of arithmetic facts, between operands and result of arithmetic facts, between arithmetic and counting facts, and within counting facts. The latter is actually 
used to produce counting errors to addition problems, though it could be argued that the pattern produced is excessively geared toward one particular error ("x is followed by x") at the exclusion of all other, perhaps more plausible ones. These interference problems will be examined in detail, the practical solutions adopted in the simulation will be reviewed, and a possible change in the architecture to address the root of the problem will be discussed.

\subsubsection{Tie Problems}

As described in the previous section, two different kinds of strengths of association will result from the associative learning mechanism applied to an arithmetic problem such as $3+4=$ ?. Positive strengths will emerge from the components of the problem $(3,+$ and 4 in this case) to the problem chunk itself, as well as other chunks containing some of the same components (e.g. $3+5=8$ ). Because those strengths converge to the empirical part of the Posterior Strength Equation, which is essentially the logarithm of a frequency ratio, their absolute value will be strongly limited (e.g. seldom higher than 2 or 3 ). The second type of strengths will occur between the components of the problems and chunks in which they do not appear (e.g. between 4 and 3+5=8). Those strengths will be initialized to 0 by the Prior Strength Equation (actually, for computational reasons the link is omitted altogether, which is equivalent), but once those chunks have been retrieved with that source in the context (e.g. $3+5=8$ being mistakenly retrieved for the problem $3+4=$ ?), a new link is created and associative learning now applies. Since retrieving that fact with that source in the context is quite rare, the resulting frequency ratio is very small, and the logarithm transformation will yield an unboundedly negative strength of association. Unlike the first type of strength which primed (i.e. raised the activation of) the chunks likely to be useful, this second type of strength will inhibit the retrieval chunks which have been a source of errors in the past. Both types of strengths of association are useful, but the latter, inhibitory type is essential in reaching a near-perfect performance in the presence of noise and partial matching, because its magnitude can grow unboundedly large very quickly due to its logarithm form. 
Tie problems (e.g. $7+7=$ ?) are unusual with respect to both types of strengths of association. As was previously mentioned, because the operand is repeated twice in the statement of the problem, its strength of association is higher. When comparing the strengths of association from a number (e.g. 7) to its tie problem (i.e. 7+7=?) and a nontie-problem (e.g. $7+8=$ ?) of similar frequency and creation time, one can see that of the quantities used in computing the empirical ratio, all are about equal ${ }^{19}$ except for the cooccurrence statistics $F\left(N_{i} \& C_{j}\right)$. These are twice as large for the tie problem because the number appears twice in context, and thus results in two co-references instead of one. Thus the strength of association to a tie problem from its operand is higher than the strength of association to other similar problems by an amount of $\ln (2)$. This factor is also reflected in the initialization of the strengths of association using the Prior Strength Equation. In the static simulations presented previously, this explained why tie problems were retrieved faster and more reliably than other problems of similar magnitude.

Tie problems are also remarkable when it comes to inhibitory connections. Other, nontie problems (e.g. $7+8=$ ?) have different operands and thus two number sources, thereby inhibiting other problems, including those on the same row (e.g. $7+9=$ ? is inhibited by 8 ) or column (e.g. $6+8=$ ? is inhibited by 7 ) of the addition table. Tie problems, on the other hand, have only one operand repeated twice and thus cannot inhibit problems on the same row or column of the addition table. The reason is that other facts $($ e.g. $7+8=15)$ are correctly retrieved quite often with their own components as sources (i.e. $7,+$ and 8), and thus will develop positive rather than negative associations from those sources. The tie problems in the same row or column of the table (e.g. $7+7=$ ?) have as sources of activation a subset of the sources for those non-tie problems (i.e. 7 and +), and thus cannot inhibit those problems.

The result is a basic asymmetry between commission errors for non-tie- vs. tie-problems. Non-tie problems (e.g. $7+8=$ ?) develop inhibitory connections to other facts, including tie

${ }^{19} F\left(C_{j}\right)$ are equal because they refer to the same source, $F\left(N_{i}\right)$ are about equal because they are of similar frequency, and $F$ are about equal because their creation times are similar 
facts involving those operands. These connections, as they become increasingly negative, prevent those facts (e.g. $7+7=14$ ) from being mismatched to that problem because the negative activation they receive becomes too much to overcome consistently with factors such as noise. Tie problems, however, cannot develop inhibitory connections to facts in the same table row or column, because those facts include as sources of activation a superset of the tie problem's sources. Therefore, even though those tie facts benefit from a small constant factor of activation boost due to the source redundancy, that is not enough to consistently overcome noise and other confusion factors. The result is that while non-tie problems asymptote toward perfect performance, tie-problems never do and even ultimately get worse as non-tie problems gradually get stronger. This is clearly an undesirable result. It should be emphasized that this is a direct result of the assumption that strengths of association from a source of activation to a chunk reflect the statistics of retrieval of that chunk given that that source is in the context, and are independent of where that source appears in the chunk if it appears at all. A version of this assumption is known in the field of machine learning as the Naïve Bayes Assumption (Mitchell, 1997), which states that the attribute values (sources of activation) are conditionally independent given the target value (chunk retrieved). It provides for a sometimes-enormous computational simplification and has been quite successful in practical applications, and indeed in past ACT-R models. However, the limits of this assumption have been exposed by the lifetime simulation because of the fundamental dependency on the learning mechanisms.

There are a number of possible solutions. An easy one would be to simply increase the Associative Learning global parameter, which controls the weight given to the prior term in the associative learning formula. This would delay the effects of the empirical ratio, including the increase in tie errors but also the emergence of strong inhibitory connections, and thus the asymptote to perfect performance for other problems. It might provide some quantitative improvement, but simply shifts the learning curve while leaving the basic problem intact. One might also argue that tie problems occur more often than others do (e.g. Ashcraft, 1992). While this might be true and would certainly help, it would only raise the base level and strengths of association to tie facts by a 
constant factor, and like the basic $\ln (2)$ effect of source duplication would not affect the long-term effect of associative learning, simply delaying it.

The solution that was adopted, as described in the first section of this chapter, consisted in explicitly recoding tie problems. When a tie problem is encountered, before a solution is attempted, the repeated argument is replaced by the special chunk Double, to represent the fact that this argument is identical to the previous one. This explicit re-encoding is consistent with ACT-R's theory of chunk creation (see Anderson \& Lebiere, 1998, pp. 23-24) because whereas chunks that result from the direct encoding of objects in the environment (e.g. 3 is the first digit) are automatically produced by the perceptual interface, chunks that originate as goals (e.g. $3+4=7$ ) are the result of explicit processing, including the serial gathering of a number of perceptual chunks. It is therefore natural that some regularities, in this case the repeating of arguments, be detected during the building of the goal chunk from perceptual chunks, and the result of this detection encoded in the goal chunk. This is consistent with the findings of Eliaser, Siegler, Campbell \& Lemaire (in preparation) that subjects spend more time encoding tie problems than regular problems. This additional time could represent the time to fire the production that detects the argument duplication. Eliaser et al. also found that non-tie problems exhibit better performance than tie problems in artificial problem sets where tie problems are the rule rather than the exception as they are in arithmetic. This suggests that the advantage enjoyed by tie problems in arithmetic in not intrinsic but instead a matter of explicit representation.

This recoding removes the duplication in the context of tie problems and provides them with a source of activation distinct from non-tie problems, i.e. the chunk Double. That chunk will develop a positive associative strength to tie problems and a strongly negative associative strength to non-tie problems because it does not appear in their formulation. This exactly provides the contextual discrimination lacking in the straightforward representation. Also, the chunk Double appears as argument in arithmetic facts about half as often as numbers do. For example, it will appear in 10 (correct) single-digit addition facts (corresponding to all the tie problems) whereas a number (e.g. 7) will 
appear as argument in 19 (10 as first argument and 10 as second argument, with the tie problem counting twice). Therefore, the positive strength of association from the chunk Double to tie problems will be about $\ln (2)$ higher in average than the strength of association from a number to a problem involving it as argument. This provides to tie problems the same advantage in associative strength that a double source did, but this time it results from an explicit recoding strategy rather than from an architectural feature.

\subsubsection{Near-tie Problems}

A related problem concerns what can be called near-tie problems, i.e. problems such as $6+7$ that are located near the diagonal in the addition table. The main problem results from argument confusion, i.e. when presented with the problem $6+7=$ ? the model often retrieves $7+6=13$ instead, because that chunk has similar base level activation and associative strengths, and the mismatch penalties (for matching 7 for 6 and vice versa) are fairly small. Since addition (and multiplication) is commutative, that results in a correct answer. The problem is that as one gets away from the diagonal (e.g. $6+8,6+9$, $5+9$, etc), this beneficial confusion effect decreases rapidly with the difference between arguments because the mismatch penalty that applies to each argument becomes too large to overcome. For example, for $9+4=13$ to match to $4+9=$ ? would require overcoming twice the dissimilarity between 4 and 9 which is much larger than between 6 and 7 . Those problems could potentially mismatch to a close fact which also yields the right answer (i.e. $5+8=13$ for $4+9=$ ?), but the likelihood of that is very small because that fact will receive no positive spreading activation, and as learning proceeds much negative activation.

The result is that problems near the diagonal (e.g. $6+7=$ ?) will tend to have lower error rates than problems of equal size and frequency further away from it (e.g. 4+9=?), a pattern that is not supported by data. This effect is particularly noticeable for large problems, whose numbers are more similar to each other (e.g. $6+7=$ ? vs. $3+4=$ ? because 6 is more similar to 7 than 3 to 4 ), and toward the end of the switch to retrieval, when those problems develop distinctive associative strengths. 
A minor additional contributor to the problem is the solution to the previous problem. Since tie facts are represented with the double chunk, they are very unlikely to intrude upon non-tie facts (e.g. $6+$ Double $=12$ will not mismatch often to $6+7=$ ? because Double is not similar to 7), and thus problems near the diagonal lose some of their closest intruders, which also lowers their error rates. However, this contribution is small and the main effect originates from the associative strengths, in particular the fact that the strengths are not slot-specific. Thus the chunk $7+6=13$ will receive the same associative strength as the chunk $6+7=13$, even though the sources appear in the chunk in the reverse order in which they appear in the retrieval template $6+7=$ ?. There is no clear solution to this problem.

\subsubsection{Corner Problems}

The previous sections dealt with the interference effects of associative strengths from the problem arguments. The slot holding the result can also produce undesirable interference effects through associative strengths. As in the previous section, those effects are most noticeable when the values interfering with each other, in this case the result and one of the operands, are very similar. That means problems in the top right and bottom left corners of the addition table, in particular problems of the form $1+x=$ ? or $x+1=$ ? for large values of $\mathrm{x}(\mathrm{e} . \mathrm{g} .1+7=8)$ because their similarities are less differentiated. In those problems, the large argument (e.g. 8 in $1+8=$ ?) will prime not only the correct fact (i.e. $1+8=9)$ but also an incorrect fact $(1+7=8)$ because that argument appears as the result. Since the mismatch penalty between large close numbers (e.g. 7 and 8) is small, this will result in an unusually large percentage of errors for those problems, which the data doesn't display.

One solution is to make sure that the associative link from a number (e.g. 8) to an addition fact mentioning it as result (i.e. $1+7=8$ ) is not reinforced. Even though a link is created by the Prior Strength Equation, it will not be reinforced by the usual addition retrieval because only the operands and operator appear as sources of activation. 
However, when an arithmetic goal (e.g. $1+7=8$ ) is popped and reinforces an identical existing chunk, the associative strengths from the arguments to that chunk must be reinforced because otherwise they will keep decaying and the chunk will never be retrieved. The solution is to have ACT-R reinforce upon merging the strengths of association from the sources of activation when the goal was created (i.e. the operands and operator), but not from the result slot value, which was filled afterward.

There are essentially two justifications for this reinforcement rule. The first is in the basic understanding of learning as improving performance in similar situations. Thus when a chunk is retrieved, the base-level activation of that chunk is reinforced, as are the strengths of association from the context chunks, all of which will improve the retrieval performance in the same context. In the case of goal merging, the similar situation is when an identical goal is created again. Ideally, that goal could be completed directly by retrieval of a past instance of that goal, instead of having to resort to less efficient means such as pushing subgoals to compute the answer. Thus the proper quantities to reinforce when a goal is popped are its base-level activation, and the strengths of association from the context chunks when that goal was created, because that is when retrieval will be needed in a subsequent episode. Reinforcing the strength of association from the result can only result in the kind of interference described at the start of this section.

The second justification arises from the nature of the sources of activation. In the current ACT-R, a chunk becomes a source of activation instantly when it becomes part of the goal, and ceases to be a slot value just as immediately when it stops being part of the goal. Lovett, Reder, \& Lebiere (in press) have argued that a more realistic model would have the sources of activation gradually accruing strength over time when they are part of the goal, with that strength decaying exponentially when they are not. Under this model, the operands and operator, which are part of the goal since its creation, will be much stronger sources of activation than the result, which only became part of the goal just before it is popped. Thus the reinforcement of the strengths of association when the goal is popped would reflect that differential source level and result in much higher strengths from operands and operator than from the result. This theory of source-level 
accumulation might also explain the need for unequal source levels noted by Anderson \& Reder (in press).

\subsubsection{Cross-type Interference}

One illustration of the undesirable effects of the type-independence assumption of associative strengths is the pattern of interference between arithmetic and counting facts. Since the vast majority (and the entirety of those modeled here) of arithmetic retrievals involves single-digit operands, the process of arithmetic retrieval creates associative interference primarily from single-digit numbers. In tasks such as addition by repeated counting, however, counting facts are retrieved for double-digit as well as single-digit numbers. This means that the pattern of errors (and latency) for counting will display a strange discontinuity: a higher percentage of errors for single-digit counting facts due to the associative interference from arithmetic facts, dropping suddenly when counting above 10 .

This pattern is of course not unavoidable and could in fact be masked by many other factors. For example, it might be that the single-digit counting facts are practiced and rehearsed, independently of their use in addition, much more often than the double-digit counting facts. That additional practice might compensate or even overwhelm the larger interference effect for single-digit facts. Or the interference effect from arithmetic retrieval for double-digit numbers might be understated. For example, retrieving those same arithmetic facts for subtraction or division purposes would entail having in the context the result as well as one of the operands, which would lead to associative interference for the result value which of course can be a double-digit number even for single-digit operands. Another possibility would be that the frequency discontinuity for arithmetic facts is artificial, either because the frequency of use of the largest single-digit facts (e.g. 9+5) is very low or because the frequency of the double-digit facts (e.g. 11+3) is higher, or both. Any of these factors, or possibly some combination of them, would obscure this particular pattern. But the fundamental problem of the improbability and undesirability of facts of very different types significantly interfering with each other 
remains. There is no obvious solution to this specific problem.

\subsubsection{A More General Solution}

It appears that the pattern of interference resulting from associative priming to a slot other than the one intended in the matching (e.g. 8 activates $1+7=8$ even though one is looking for a chunk matching $1+8=$ ?) is generally undesirable, as is the interference resulting from the statistical patterns of use of other chunk types (e.g. limiting addition facts to single digits makes counting up single digits harder than counting up double digits). What seems to be needed is a way to make associative connections slot- and typespecific.

One could make the frequency statistics used in the empirical ratio specific in that way, but the record-keeping then becomes very onerous and the statistics less reliable because separate values have to be kept for each type and slot. ${ }^{20}$ It also leads to occasionally counterintuitive predictions, for example that, assuming equal fans, the prior strength of association (as determined by the Prior Strength Equation) to a chunk of a certain type would be larger than the prior strength of association to a chunk of another type if there were more elements of the former type. That seems clearly undesirable: all things being equal, more chunks of a certain type ought to mean more interference, and thus worse performance, not the better performance resulting from stronger strengths of associations.

More generally, maintaining slot- and type-specific connections seems generally unworkable. For example, the same number (e.g. 7) could develop both a positive strength of association to a chunk (e.g. $7+8=$ ?) because it appears as a slot value (i.e. the first operand) when the chunk is retrieved but also a negative strength of association because the chunk is occasionally mismatched when it appears in another slot (e.g. $7+7=$ ? where it appears as the second operand). Thus the sources of activation would not be chunks but chunk-slot combinations. In addition to the increased computational expense

\footnotetext{
${ }^{20}$ These are the same reasons for why the Naïve Bayes Assumption is so popular in
} 
of keeping those additional links, this is clearly at odds with the current concept of strengths of association between chunks, and would lead to a very odd implementation.

A more elegant solution might be to consider the duality between the processes of activation spreading and partial matching. For example, given a retrieval from the goal pattern of $3+4=$ ?, the activation spreading will raise the activation of chunks that match that pattern (contain the sources), and the partial matching will lower the activation of chunks that mismatch the pattern. Clearly the two mechanisms are performing related functions and could potentially be unified. One interesting difference is that while activation is spread to all the chunks to which the source is connected, the partial matching is type-specific (because only chunks of the right type are allowed to match) and slot-specific (the desired slot value is matched against the actual chunk slot value, independently of the other sources appearing in the other slots). This is precisely the solution to the problems with the strengths of association described in the previous sections. Could the solution be simply to remove the activation spreading and rely on partial matching to provide context-specificity?

This would certainly be compatible with ACT-RN (Lebiere \& Anderson, 1993), an earlier implementation of ACT-R using standard neural network constructs, which has guided the development of ACT-R including the partial matching mechanism (Lebiere, Anderson, \& Reder, 1994). In ACT-RN, the concepts of strengths of association and partial matching are not separate. The pattern of connections to a chunk from its slots encode both the association from slot value to chunk, and the distributed pattern of activation of the slot value which allows different but similar values to capture part of the strength of association.

One function of the associative learning mechanism which cannot be readily performed by the partial matching is the gradual discrimination between associative strengths that occurs over time and leads to increasingly negative strengths of association which result in near-perfect performance. What could change over time that would allow the partial

machine learning. 
matching mechanism to initially produce the error-prone performance of children and later improve to the near-flawless performance of adults? One possibility would be the similarity values. Smaller, more frequently encountered, numbers are usually considered less similar than larger, less common numbers (Whalen, 1996). While the better discriminability between small numbers could be due to their magnitude itself (i.e. two apples look more different from three than seven from eight), it could also be attributed to their higher frequency. The ability to discriminate through similarity values between related concepts might be acquired through practice. This might explain the improved performance over time as similarity values are increasingly refined and differentiated and errors of commission are gradually reduced.

Another, perhaps more promising possibility would be the activation noise. ACT-R's assumption of an activation noise of constant amplitude is at odds with the intuition that strong, well-established chunks ought to be less noisy than new, poorly rehearsed chunks. $^{21}$ If the amplitude of the activation noise was specific to a chunk and decreased with the amount of practice of that chunk, then the commission errors involving that chunk would decrease along with the noise since one term would ultimately come to dominate the Boltzmann equation as temperature goes down to 0. If one interprets the decay of base-level activation as being a stochastic phenomenon, this decrease of noise with practice would explain how decay becomes increasingly slow with practice. One possible function expressing the noise $S_{n}$ as a function of practice $n$ and the initial noise $S$ is:

$$
S_{n}=\frac{S}{1+\ln (n)}
$$

\section{Noise Reduction Equation}

Let us assume for simplicity that chunks $C_{1}$ and $C_{2}$ are competing for retrieval and that

\footnotetext{
${ }^{21}$ ACT-R 4.0 introduced a Permanent Activation Noise sampled once at the creation of the chunk, which is distinct from the Activation Noise added to the chunk activation at each production cycle and usually of larger amplitude to provide greater initial randomness. This however would not be helpful in this model.
} 
they have had a similar number of retrievals $n$, then the odds of retrieving chunk $C_{1}$ are:

$\operatorname{Odds}\left(C_{1}\right)=e^{A_{1}-A_{2} / S_{n}}$

If one expressed the noise level as a function of practice, the odds of retrieval become:

$\operatorname{Odds}\left(C_{1}\right)=e^{(1+\ln (n))\left(A_{1}-A_{2}\right) / S}=e^{A_{1}-A_{2} / S} \cdot n^{A_{1}-A_{2} / S}$

This establishes that if one assumes that the noise level decreases as an inverse function of the logarithm of practice, then the odds of retrieval (i.e. error) follow a power law of practice, with the exponent being the ratio of the activation difference between the competing chunks, divided the amplitude of the initial noise. The speed of convergence will of course improve with lower noise, but also with wider activation separation between chunks. This activation difference can result from a difference in frequency of practice or rehearsal between chunks, as well as from activation penalties resulting from chunk(s) mismatches. A higher mismatch penalty will therefore also lead to faster convergence.

Decreasing the noise of chunks over time with their amount of practice is closely related to the technique of simulated annealing in Boltzmann machines (Ackley, Hinton, \& Sejnowski, 1985; Hinton \& Sejnowski, 1986), since noise in ACT-R has an effect similar to temperature in Boltzmann machines, through the same Boltzmann equation. However, there are differences as well. The Boltzmann distribution in ACT-R is merely descriptive, whereas it is also used in Boltzmann machines to control every local unit fluctuation. More fundamentally, simulated annealing in Boltzmann machines happens on a small time scale, for every pattern presentation, whereas it would be under this proposal a long-term process, with the activation noise decreasing over a long time scale with each rehearsal. Finally, temperature in Boltzmann machines is a quantity global to the entire network, whereas every chunk in ACT-R would have a different noise as a 
function of its amount of practice, with well-settled knowledge being gradually frozen in place but more recent knowledge showing significant fluidity. Another analogy might be made to the Gibbs Sampler of Geman \& Geman (1984), who show that the fastest annealing schedule assured to converge to the energy minimum is of the form given by the Noise Reduction Equation. They also show that the energy minimum corresponds to the maximum a posteriori (MAP) estimate of the underlying distribution, providing an alternative interpretation of ACT-R's Bayesian learning mechanisms.

\subsection{Retrieval as Subgoaling}

As developed in the previous sections, the strengths of association become increasingly differentiated as the simulation progresses. The strengths of association between numbers and related facts (e.g. 3 and $3+4=7$ ) become large positive numbers, and the strengths of association between numbers and unrelated facts (e.g. 5 and $3+4=7$ ) become increasingly and unboundedly negative. The latter results from the increasingly infrequent retrieval of the fact given the unrelated number, and is the essential condition to achieving asymptotically perfect performance given initial error-prone performance.

However, in complex computational goals such as to perform addition by repeated counting and multiplication by repeated addition, the source activation will be divided among many sources such as counters and intermediate results, only a subset of which are involved in any given retrieval. For example, the following goal to compute the sum of 4 and 3 by iterative counting starts as:

\section{Goal}

isa iterate-count

count 0

limit 3

result 4

The first step will be to increment the intermediate result 4 and counter 0 by retrieving the appropriate counting facts. However, if those retrievals are done directly, then 0,3 
and 4 will all be sources when the counting facts $0->1$ and $4->5$ are retrieved. The strengths of associations between those numbers and facts will all be reinforced, including incidental ones such as between 4 and $0->1$, and 3 and 4->5. This would have two undesirable consequences. The first is interference errors, such as retrieving 3->4 instead of 4->5 because the extra spreading activation from the source 3 overcomes the mismatch penalty between 3 and 4 . While the errors produced by this type of interference do occur, in this case the interference leads to an excessively deterministic pattern of errors. An even more fundamental problem is that because of these accidental reinforcements between unrelated facts and numbers, the strengths of association between them will not become increasingly negative but instead settle at some base value that reflects these accidental co-occurrences and that will prevent further improvement in performance.

The solution is to subgoal retrievals instead of performing them directly. This corresponds to moving the retrievals from the left-hand side of productions to the righthand side and pushing subgoals to perform them on the stack. This operation focuses on the retrieval to be performed by creating a new goal of the same type as the chunk to be retrieved and which only includes the activation sources necessary to the retrieval. Examples of this technique can be found in productions Iterate-Count (which subgoals a count) and Iterate-Add (which subgoals a number of operations). Once the retrieval patterns have been subgoaled, a production must fire to perform the actual retrieval, complete the pattern, and pop the goal. These productions are Count-Up and Arithmetic-Retrieval for counting and arithmetic facts, respectively. These productions, which complete a goal by matching it to a chunk in memory, are very basic and can be found in many other models (e.g. Lebiere \& Wallach (in preparation)). Indeed, these productions are so basic and pervasive that it could be argued that they correspond to an architectural primitive similar to the Obligatory Retrieval Assumption of Logan (1988).

This technique, in addition to allowing the strengths of association to achieve optimal predictiveness, has the advantage of increasing the modularity of knowledge. Instead of having to generate separate productions for retrieval and backup computation in each 
situation in which an arithmetic fact would be needed, a single production would be needed to set up a subgoal of that type, which could be solved by either retrieval or backup computation without the need to duplicate those productions. By pushing a subgoal that will become a declarative memory chunk when it is popped, it also creates a permanent record of the retrieval in memory which allows the system to reflect upon its own problem-solving. It would also improve retrieval performance, because the entire source level W would be devoted to sources useful to retrieval, and thus would speed up learning as well by limiting errors.

Finally, it would make for a much simpler implementation of ACT-RN (Lebiere \& Anderson, 1993). In ACT-RN, productions perform retrievals by gating the right connections between central memory, in which the current goal is held, and declarative memory, which is implemented as a set of type memories, each of which holding chunks of a different type. Because any goal can perform any retrieval, the mapping between goal values and retrieval pattern is arbitrary. Thus productions must have gating connections to enable any possible permutation from central memory to any type memory. Because declarative memory is potentially quite vast, this generality is very computationally expensive. This proposal offers a much more manageable solution. Because a retrieval is accomplished by first creating a new subgoal, that transformation can be performed by local connections within central memory. Since the new subgoal directly specifies the retrieval pattern, the actual retrieval can then be performed by directly broadcasting the subgoal to declarative memory, without the need for any gating connection to specify the retrieval pattern or the right type memory. Since retrieval latency is inversely proportional to degree of match, the type memory holding the chunk that best matches the subgoal will complete retrieval first and preempt the other memories in returning the resulting chunk to central memory. This broadcast communication between the central controller executive and the declarative memory modules is similar to the CAP2 connectionist control architecture (Schneider \& Oliver, 1991; Schneider \& Pimm-Smith, 1997). 


\section{Chapter 5: Parameter Sensitivity}

\subsection{Introduction}

The simulations described here are controlled by a number of real-valued parameters. The values of those parameters were selected (optimized would imply more precision and systematicity than could be practically used considering the length of the simulation) to maximize the fit to the experimental data. A common criticism of ACT-R is that it contains too many parameters that can be used to tune every aspect of the system. ${ }^{22}$ While factually correct, this critique mischaracterizes the impact that those parameters have upon the predictions of a particular model. Clearly some amount of parameterization is needed in any architecture to account for individual and experimental variations, estimation of unknown variables (previous knowledge, etc) and the like. While varying parameter values within a reasonable range will result in different quantitative predictions, the model's qualitative predictions are left unaltered. For example, if one assumes a differential in frequency between small and large facts, any reasonable ACT-R model will yield a problem-size effect. This is a basic prediction of the architecture's learning mechanism.

Moreover, there has been recently a concerted effort (Anderson \& Lebiere, 1998) to understand the effect and constrain the values of ACT-R's global parameters, i.e. those parameters that are specified in the architecture and apply to every model. Some parameters, such as the noise level, have become increasingly constrained to a narrow range of values. Others, such as the base level decay, have essentially been fixed. Even those parameters whose values vary more widely, such as the retrieval threshold and latency factor, can be related to each other through known principles such as the speedaccuracy tradeoff (Anderson, Bothell, Lebiere, \& Matessa, 1998), thereby removing

\footnotetext{
${ }^{22}$ Strangely enough, those critics are often unperturbed by connectionist models, which by definition consist of little else but parameter estimation, automated and otherwise.
} 
further degrees of freedom.

This chapter will examine the sensitivity of the model to its parameters, including global parameters such as the activation noise, retrieval threshold, and mismatch penalty, as well as domain-specific parameters such as training schedule, problem distribution, and feedback strategies. Beyond establishing that the model is not excessively sensitive to any parameter value and that the results obtained qualitatively hold for a wide range of values, the goal is to explore the concept of optimality of those parameters, not in terms of fitting the data but in terms of absolute performance. The concept of optimal values for the simulation-specific parameters is fairly intuitive as well as practical: given a particular cognitive system (children, ACT-R model, etc), what is the best way to teach it arithmetic in terms of how fast to present the problems, how much feedback to give, etc. Those are questions whose answers would be of some use to educators. The concept of optimal values for the global parameters of the cognitive system is somewhat different.

One would be tempted to say that the optimal noise value is 0 (so everything is precise and deterministic), the retrieval threshold is minus infinity (so every chunk is perfectly recalled) and the optimal mismatch penalty is infinity (so every chunk is perfectly matched and no error occurs). This essentially corresponds to the purely symbolic mode of execution of ACT-R, but it represents an overly simple view of cognition. Noise is useful because it promotes adaptation to a constantly changing environment. A retrieval threshold is useful because it provides a criterion to establish when no suitable memory can be retrieved and some alternative strategy must be used. Partial matching is useful because it provides an ability to generalize by retrieving related information when the exact match does not exist or is not available. But clearly there must be a limit. If the noise is too high the system is random. If the retrieval threshold is too high, declarative memory is useless. Too much mismatching and confusion sets in. There arises the concept of an optimal value for activation noise, retrieval threshold and mismatch penalty. But of course such a concept would have to be environment-specific. Stochasticity, thresholding and approximation are more likely to be useful in an approximate, rapidly changing environment. And conversely, they are less likely to be 
useful in a domain such as cognitive arithmetic, which, with its immutable set of facts, puts a high premium on precision. But it would nonetheless be useful to establish that those attribute of human cognition, which have evolved in a very different environment than the precise world of mathematics, are still of some use there. The following sections will illustrate that this is indeed the case, to a surprising extent. Because of the need to run the lifetime simulation in each study for a range of parameter values, each data point represents a single simulation run.

\subsection{Activation Noise}

The formal analysis established that the activation noise is the main parameter controlling the speed of convergence to correct fact retrieval. Figure 5.1 presents the results from running the lifetime simulation for a range of activation noise $s$ values from 0.0 to 0.5 , with all other parameters held constant.

\section{Odds of Retrieval Error as a function of Activation Noise $\mathbf{S}$}

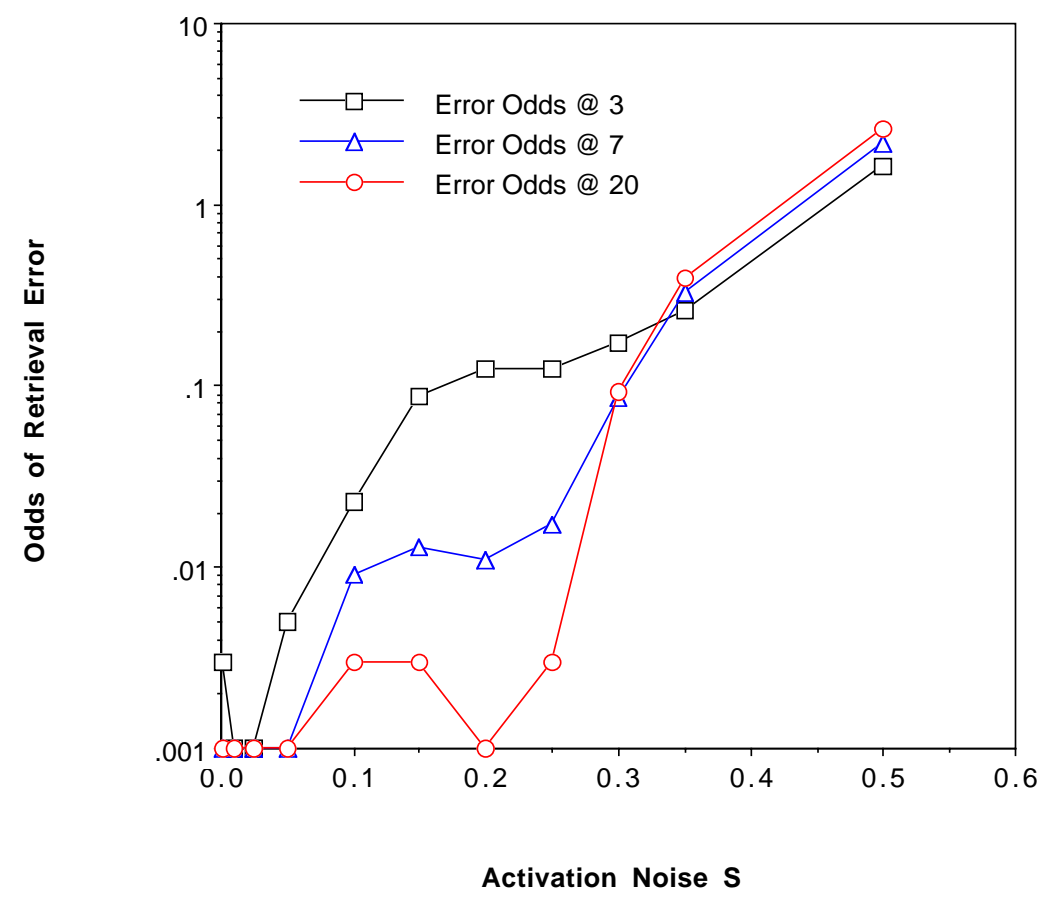

Figure 5.1: Odds of Retrieval Error as a function of Activation Noise S. 
The results are presented for 2000-problem cycles 3, 7 and 20, corresponding roughly to second grade, sixth grade and adulthood. The odds of retrieval error are plotted on a log scale as a function of the activation noise. The results clearly confirm the theoretical analysis. For values of $s$ of 0.25 or smaller, the odds of error decrease to about $1 \%$ or less by cycle 7 and to a negligible amount by cycle 20 . For larger noise values, the error odds either stabilize, e.g. to about $10 \%$ for $s=0.3$, or even increase over time, e.g. to worse than even odds for $s=0.5$. The theoretical analysis predicted that the noise threshold for convergence would be 1 divided by $\sqrt{ } 2$ (because commission errors result from the combined noise of two chunks) times 3 (because the source activation level for each argument is $1 / 3$ ), or about 0.236 . The actual threshold is slightly higher, around 0.25 , because other factors such as corrective feedback and mismatch penalty also contribute to reducing the odds of error.

While this would argue for a noise level as low as possible to ensure the fastest convergence to the correct answer, other factors enter into a measure of optimality. One is that very low noise levels occasionally lead to some error becoming ingrained and preventing convergence to perfect performance because the system is not stochastic enough to escape the problem. A more fundamental factor is the desirability of retrieving an answer instead of computing it or relying on external help, which can be timeconsuming and error-prone. Figure 5.2 plots the odds of retrieval at cycles 1, 2 and 3 as a function of the activation noise level. The odds of retrieval increase roughly as an exponential function of the activation noise, with the slope of the curve gradually decreasing over time to about even at cycle 3 . In the early cycles, when the activation level of most chunks is below the retrieval threshold, the larger the noise the more likely a chunk is to be retrieved. Thus a large noise boosts the odds of retrieval and makes it possible for the model to switch to retrieval earlier, albeit with more errors. This earlier switch to retrieval will lead to additional reinforcements from the retrieval process itself, and will in turn result in not only more common but also faster retrievals, as indicated by Figure 5.3, which plots the retrieval latency for small and large facts at cycle 7. A higher noise leads to faster retrieval, especially for large facts. 


\section{Retrieval Odds as a function of Activation Noise S}

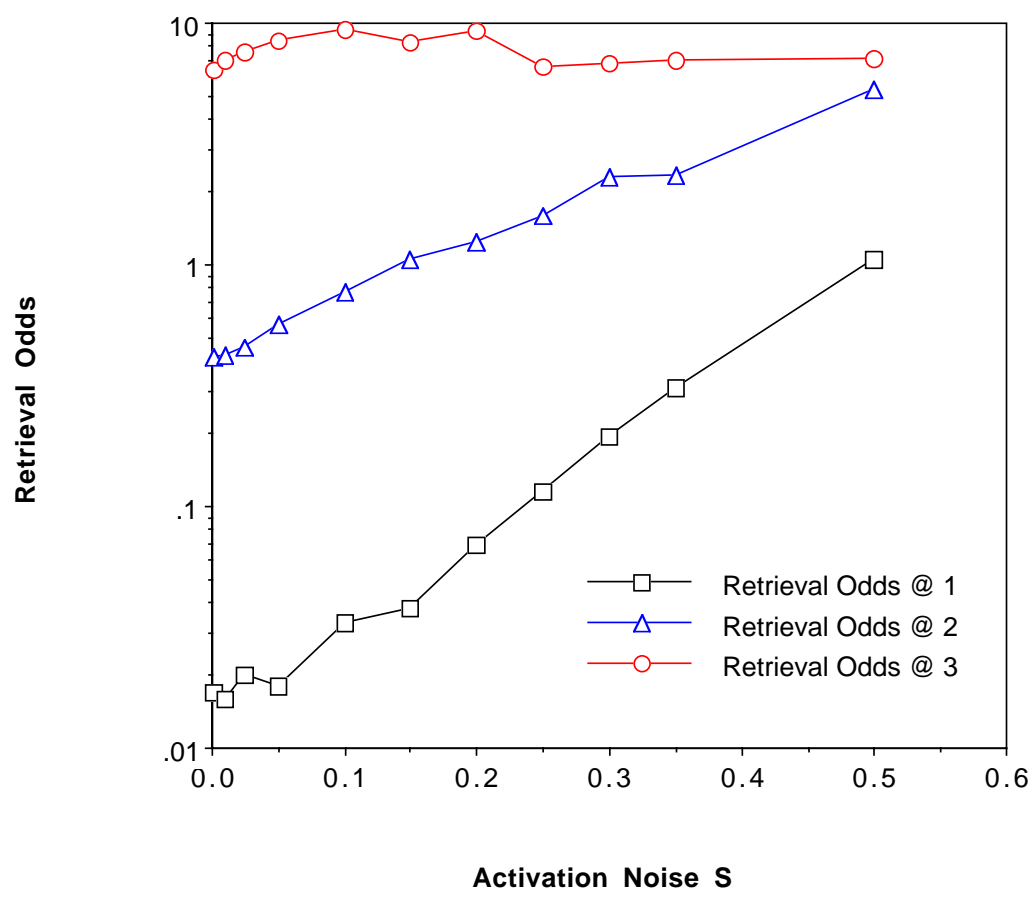

Figure 5.2: Odds of Retrieval as a function of Activation Noise S.

\section{Retrieval Latency as a function of Activation Noise S}

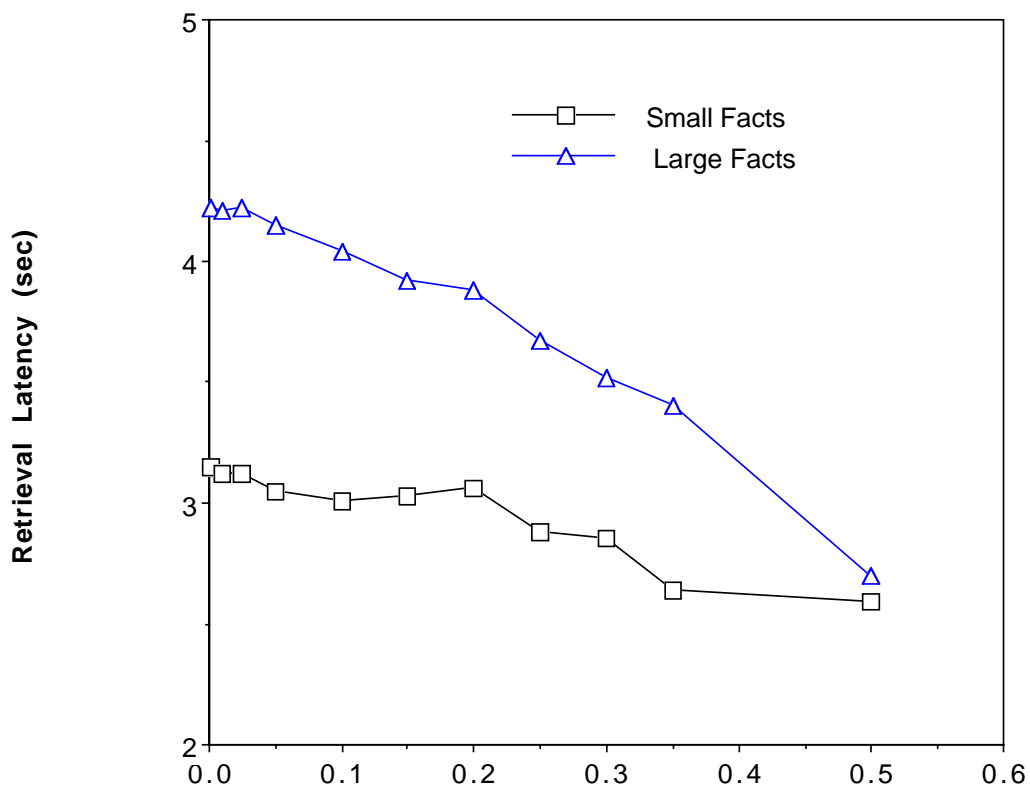

Activation Noise $\mathbf{S}$

Figure 5.3: Retrieval Latency as a function of Activation Noise S. 
Thus one could say that by some measure the lifetime simulation noise level of 0.25 is about optimal, in that it provides the earliest and fastest possible arithmetic retrieval under the constraint of ensuring convergence to the correct answers. And this optimality criterion is fairly strict. Because odds of retrieval error increase exponentially with the noise level, a significantly larger noise value will lead to an unacceptably large proportion of errors that will not be corrected with practice. And because the odds of retrieval initially decrease as an exponential of the activation noise, a much lower noise value will lead to a much slower initial shift to retrieval. Unlike the values of the retrieval threshold which tend to vary more widely, this value of 0.25 for the activation noise has been used in other simulations (e.g. Lebiere \& Wallach, in preparation; West \& Lebiere, in preparation) and has become something of a recommended setting for the noise level.

\subsection{Retrieval Threshold}

A priori, it doesn't appear that the retrieval threshold would have much impact on the probability of error. Clearly, through the Retrieval Probability Equation the retrieval threshold directly determines the probability of a chunk being retrieved, i.e. the odds of retrieval are:

$$
O d d s=e^{A-\tau / s}
$$

This inverse exponential relation is confirmed by plotting the log odds of retrieval as a function of retrieval threshold for the first 3 cycles in Figure 5.4. Thus a lower threshold provides for a much faster initial switch to retrieval. However, a very fast transition to retrieval is problematic. If some chunks are being consistently retrieved and are building up strength while other neighboring chunks haven't yet started being retrieved, there is a danger of the stronger chunks being misretrieved for the weaker ones, thus getting even stronger, and increasingly getting locked in as permanent errors in a vicious rich-getricher circle. To resist that phenomenon, one needs a gradual and relatively uniform 
building of base level and associative strengths before retrieval triggers its own dynamic feedback loop.

Odds of Retrieval as a function of Retrieval Threshold

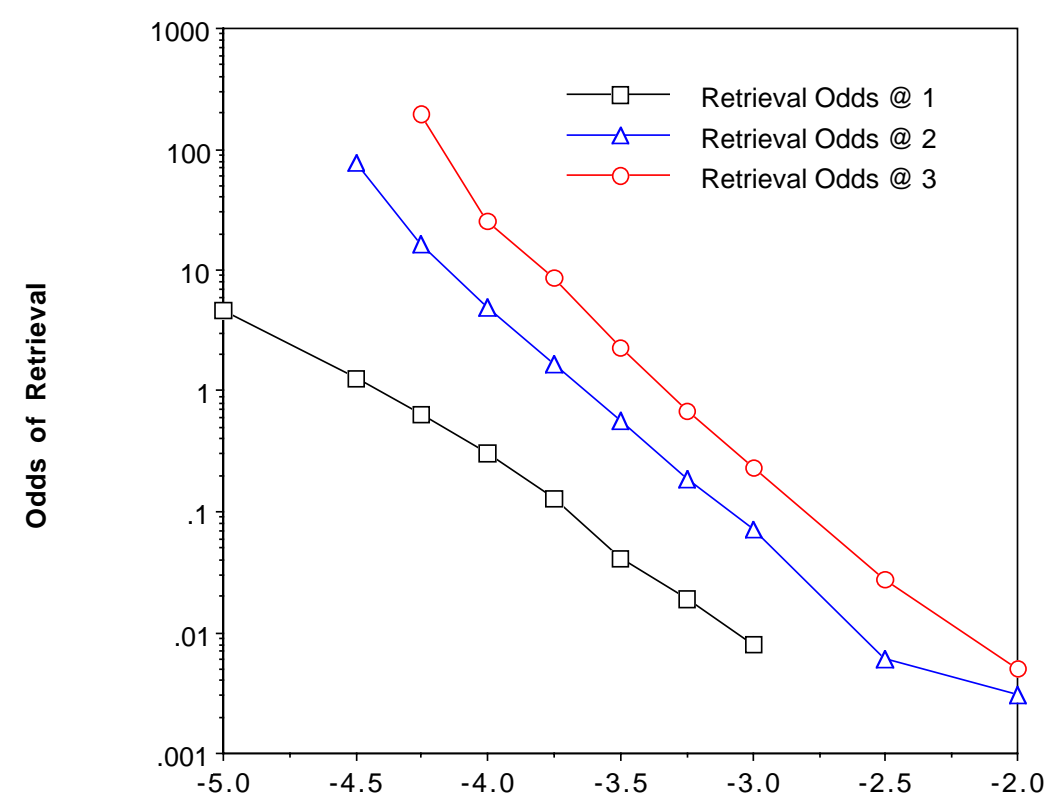

Retrieval Threshold

Figure 5.4: Odds of Retrieval as a function of Retrieval Threshold.

This analysis is confirmed by the empirical results presented in Figure 5.5, which plots the odds of retrieval error on a log scale as a function of retrieval threshold at cycles 3,7 and 20. One can observe a sharp exponential degradation in the odds of retrieval error below a threshold of -3.75 , the retrieval threshold used in the lifetime simulation. For a slightly lower threshold of -4.0 , the percentage of errors stabilizes at about $5 \%$, whereas for even lower thresholds down to -5 the odds of error actually increase over time to even odds or even much worse. This dynamic is reminiscent of the effects of large activation noise values.

However, threshold values larger than -3.75 do not yield better performance. The odds of error again increase exponentially, though at a much slower pace, and ultimately decrease over time at the same rate as the optimal value, indicating a different problem 
than for lower values. The reason for the poorer performance of larger threshold values results from the use of backup computations: since those strategies have a high error rate, they generate many erroneous facts that will degrade the retrieval performance later on. The slope of this performance degradation will depend upon the degree of reliance upon error-prone backup strategies (versus error-free but potentially burdensome strategies such as asking a teacher or parent), and convergence to error-free performance will only be delayed rather than prevented. However, this clearly establishes that delaying retrieval with further practice is not productive, and clearly establishes an optimal value for the retrieval threshold.

\section{Odds Of Retrieval Error as a function of Retrieval Threshold}

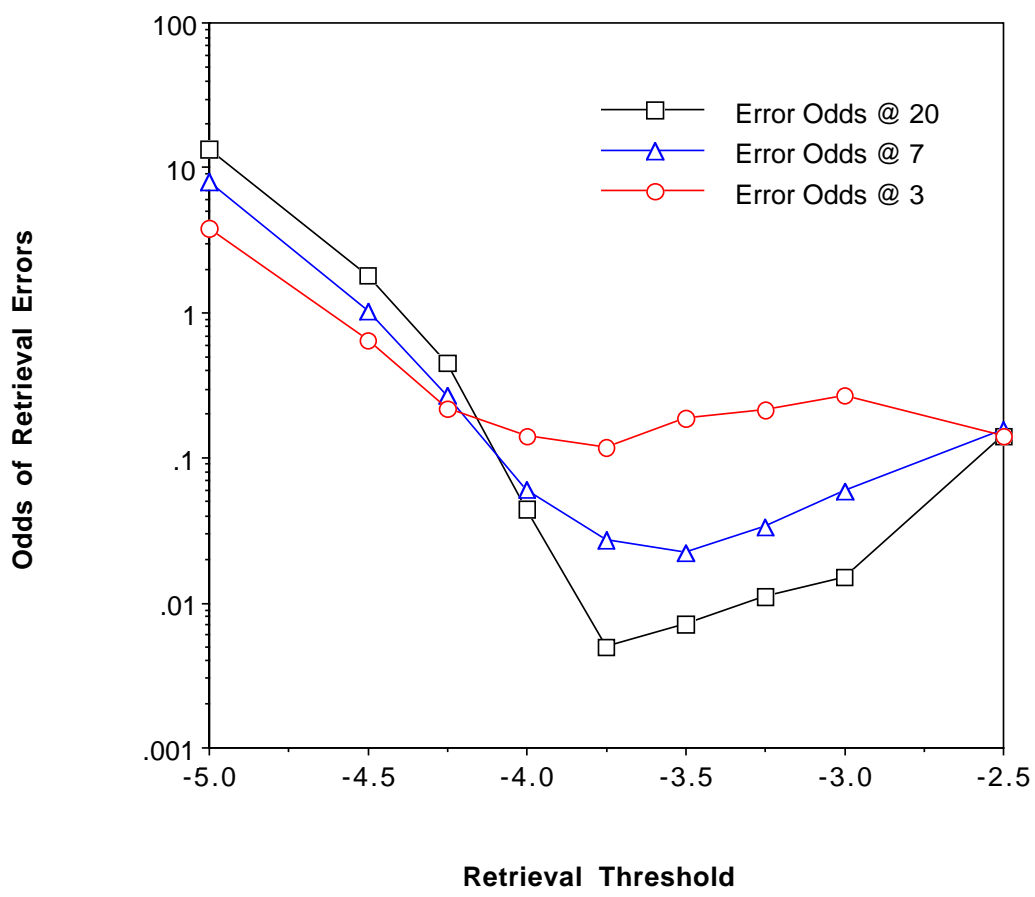

Figure 5.5: Odds of Retrieval Error as a function of Retrieval Threshold.

In addition to leading to a sharp increase in errors, a very low threshold also leads to a very strange latency pattern, as shown in Figure 5.6 plotting the retrieval latency for small and large facts in cycle 7 as a function of retrieval threshold. 


\section{Retrieval Latency as a function of Retrieval Threshold}

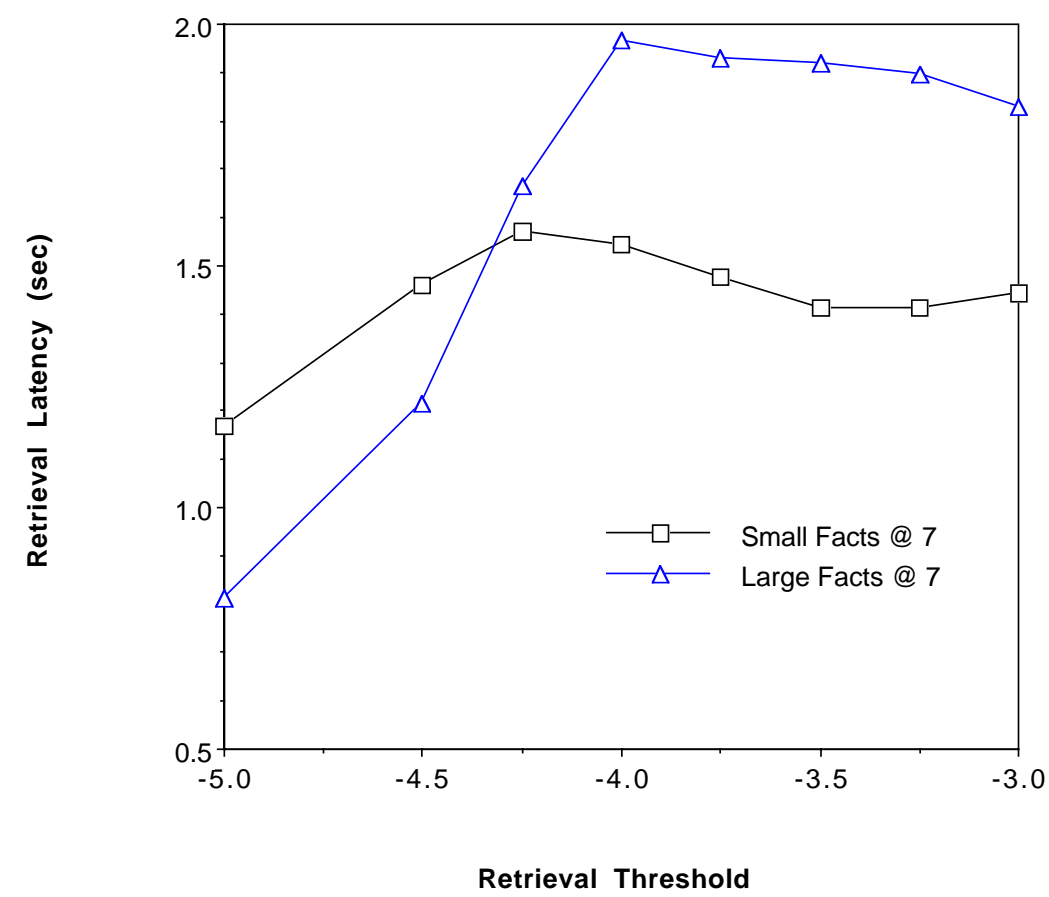

Figure 5.6: Retrieval Latency at cycle 7 as a function of Retrieval Threshold.

Whereas for a retrieval threshold larger than -4.0 , the expected problem-size effect between small and large fact retrieval can be observed, for lower thresholds the problemsize effect gradually becomes inverted. This is a rather complicated consequence of the increase in associative strength magnitude with number size described in section 4.1, combined with the rapid emergence of a few very strong facts at very low threshold. While somewhat obscure and secondary, this effect reinforces the conclusion that while they might seem attractive very low thresholds can be disastrous in practice. In conclusion, similarly to the activation noise, a strongly optimal value for the retrieval threshold exists due to the sharply exponential increase in errors for lower thresholds and the much slower switch to retrieval and slighter increase in errors for higher thresholds.

\subsection{Mismatch Penalty}

Since the mismatch penalty scales the difference in activation between the correct fact 
and potential mismatching competitors, one would expect an exponential decrease in errors to result from an increasing mismatch penalty. This is confirmed by the empirical results of Figure 5.7, which plots the odds of retrieval error as a function of the mismatch penalty parameter at cycles 3,7 and 20 .

\section{Odds of Retrieval Error as a function of Mismatch Penalty}

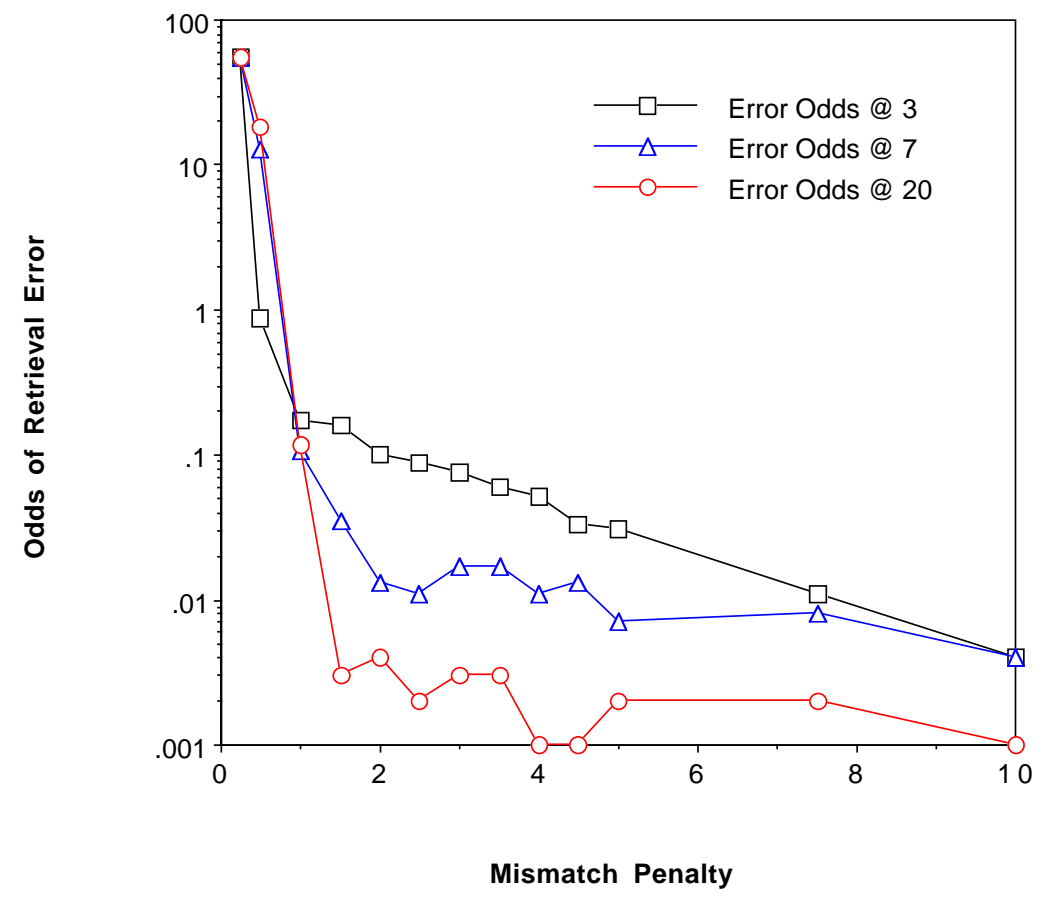

Figure 5.7: Odds of Retrieval Error as a function of Mismatch Penalty.

A similar pattern as for activation noise and retrieval threshold again emerges, with the threshold value for mismatch penalty being the default one of 1.5. Slightly lower values such as 1.0 result in a stable retrieval error percentage of about 10\%. Significantly lower values lead to a very sharp degradation in performance with error rates increasing over time to catastrophic levels. A much more gradual increase in performance occurs for mismatch penalty values greater than the threshold, with uniform convergence over time to perfect performance.

One could conclude that larger mismatch penalties are more desirable, and the exactitude 
and monotonicity of arithmetic knowledge makes it one of the most propitious domains for that assumption. True to the emerging pattern, excessive insistence upon exactitude, however, will also lead to a slower shift to retrieval, as shown by Figure 5.8, which plots the odds of retrieval for cycles 1 to 3 as a function of the mismatch penalty:

\section{Odds of Retrieval as a Function of Mismatch Penalty}

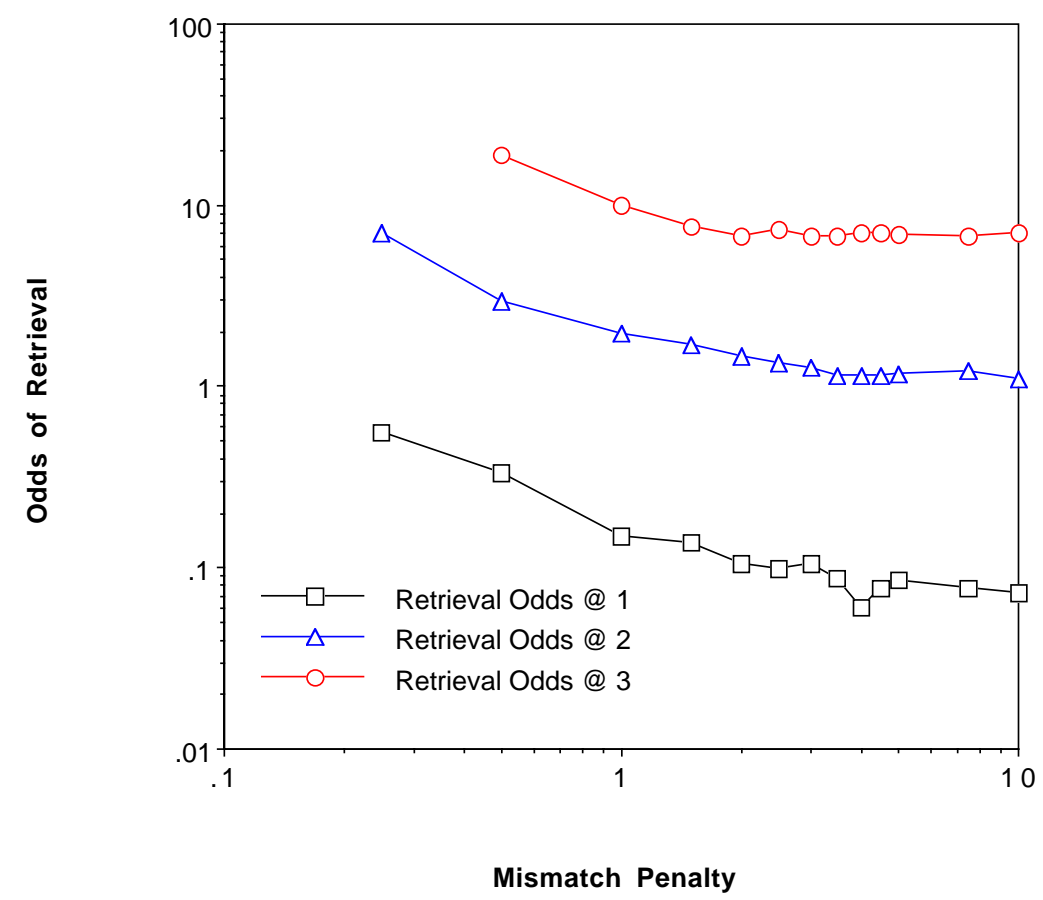

Figure 5.8: Odds of Retrieval as a function of Mismatch Penalty.

There is to a slight but discernible increase in speed of retrieval with decreasing mismatch penalties. This results because decreasing mismatch penalties will increase retrieval errors. However, incorrect retrievals still qualify as rehearsals for the facts to which they correspond and, at least in the early cycles, the feedback correction gives the correct fact the same rehearsal that a correct retrieval would have provided. Thus incorrect retrievals actually lead to more rehearsals and more retrievals. In conclusion, an optimal value again emerges which provides for the fastest switch to retrieval under constraint of convergence to the correct answers. 


\subsection{Presentation Schedule}

In addition to the architectural parameters examined in the previous section, the behavior of the lifetime simulation is controlled by a number of parameters specific to the domain of cognitive arithmetic. One of these is the presentation schedule. While many variations are possible, the most straightforward manipulation is to vary the average time delay between problem presentations. One would expect that shorter delays, i.e. a more intense schedule, would lead to faster and better retrieval, and the former is certainly correct, as evidenced by Figure 5.9, which plots the odds of retrieval for cycles 1 to 3 as a function of inter-problem delay:

\section{Odds of Retrieval as a function of Inter-Problem Delay}

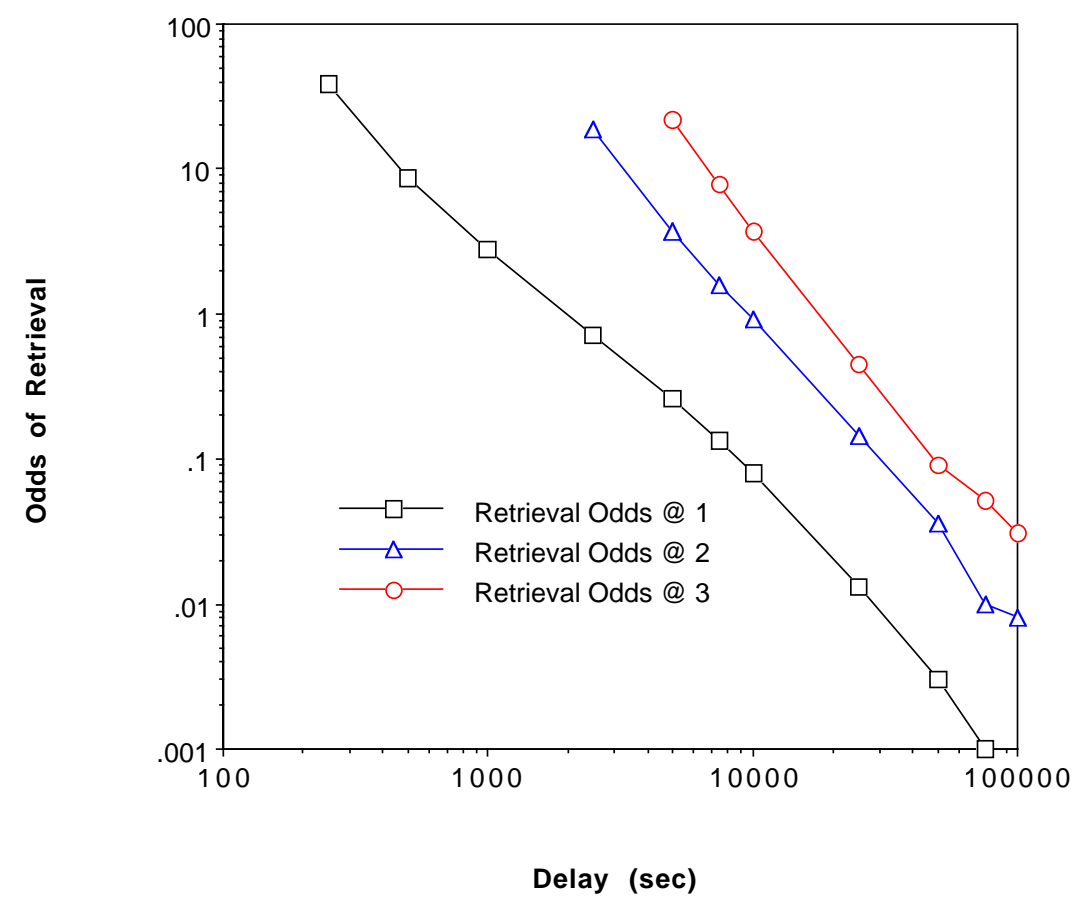

Figure 5.9: Odds of Retrieval as a function of Inter-Problem Delay.

One can see that the odds of retrieval decrease as a power function of the inter-problem delay with an exponent of about -2 . This can be derived by replacing in the approximate form of the Base-Level Learning Equation the total life of the chunk $T$ with $n . l$ where $n$ is the number of references and $l$ is the lag or inter-problem delay to obtain: 


$$
B=\ln \frac{n^{1-d} \cdot l^{-d}}{1-d}
$$

This new expression of the base-level activation can then be used in the odds version of the Retrieval Probability Equation to yield, using $C$ to abstract away the constant activation terms:

$O d d s=C \cdot e^{-d \ln l / s}=C \cdot l^{-d / s}$

which confirms not only the power-law form of the relation between odds of retrieval and inter-problem delay, but also the exponent value of about -2 , since the base-level decay exponent $d$ is 0.5 and the activation noise level $s$ is 0.25 . Thus a smaller inter-problem delay, hence a more intense schedule, leads to faster retrievals, but not necessarily better retrievals, as Figure 5.10, which plots the odds of retrieval error as a function of interproblem delay for cycles 3, 7 and 10. Again an optimal value emerges at about 7500 seconds of inter-problem delay, the value used in the lifetime simulation. For shorter delays, the performance quickly degrades and actually gets worse over time. A slightly shorter delay of 5000 seconds is initially slightly better but doesn't seem to converge as well. Somewhat larger delays up to 25000 seconds are initially slightly worse but converge as well as the optimal value, but of course they take up to three times as long as the optimal value since the total time scales linearly with the delay. Even larger delays start and stabilize at about $10 \%$ errors.

The explanation is similar to that for the retrieval threshold, since the main effect of interproblem delay is to uniformly raise or lower the base-level activation, which is equivalent to respectively lowering or raising the retrieval threshold. Very low inter-problem delays will lead to a quick buildup of base-level activation and thus an early retrieval of chunks, which results in a catastrophic performance degradation as a few chunks overwhelm the others in a rich-get-richer grab. Larger inter-problem delays are safer, but result in more frequent uses of the backup computation procedure, which introduces additional errors. 


\section{Odds of Retrieval Error as a function of Inter-Problem Delay}

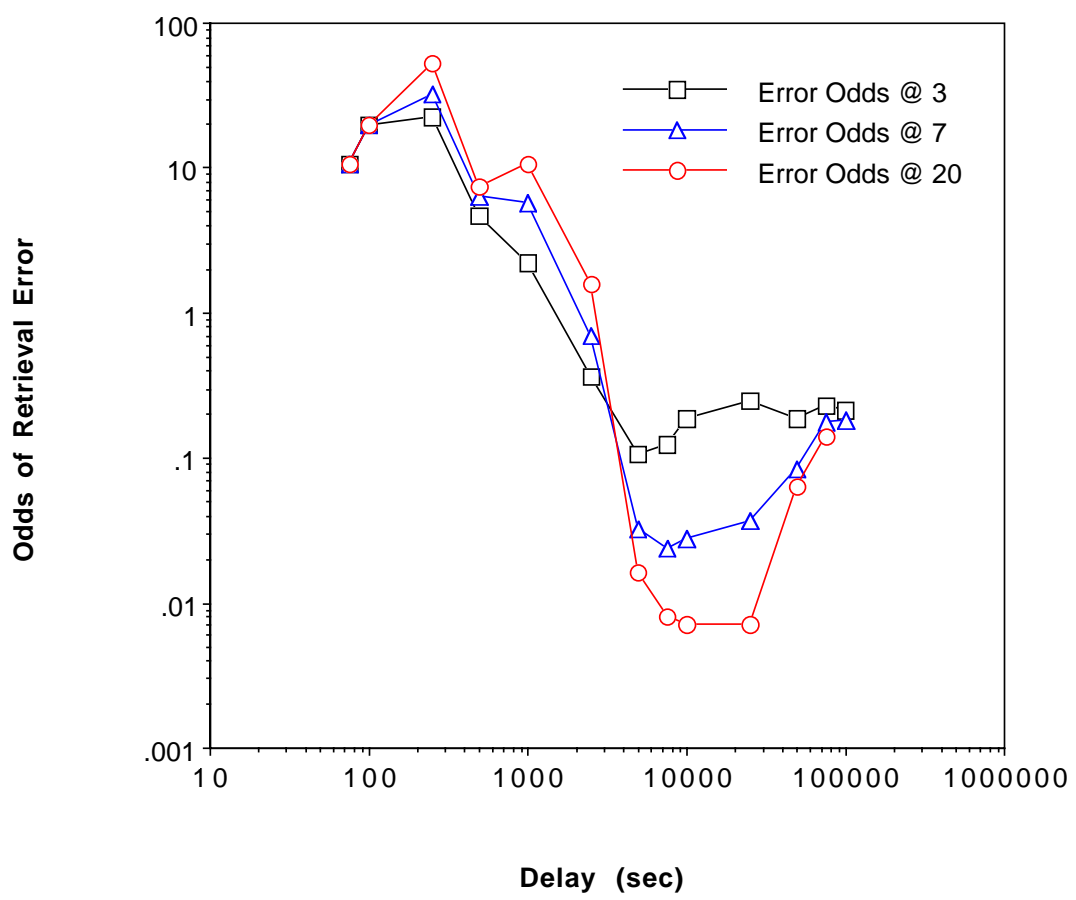

Figure 5.10: Odds of Retrieval Error as a function of Inter-Problem Delay.

\section{Retrieval Latency as a function of Inter-Problem Delay}

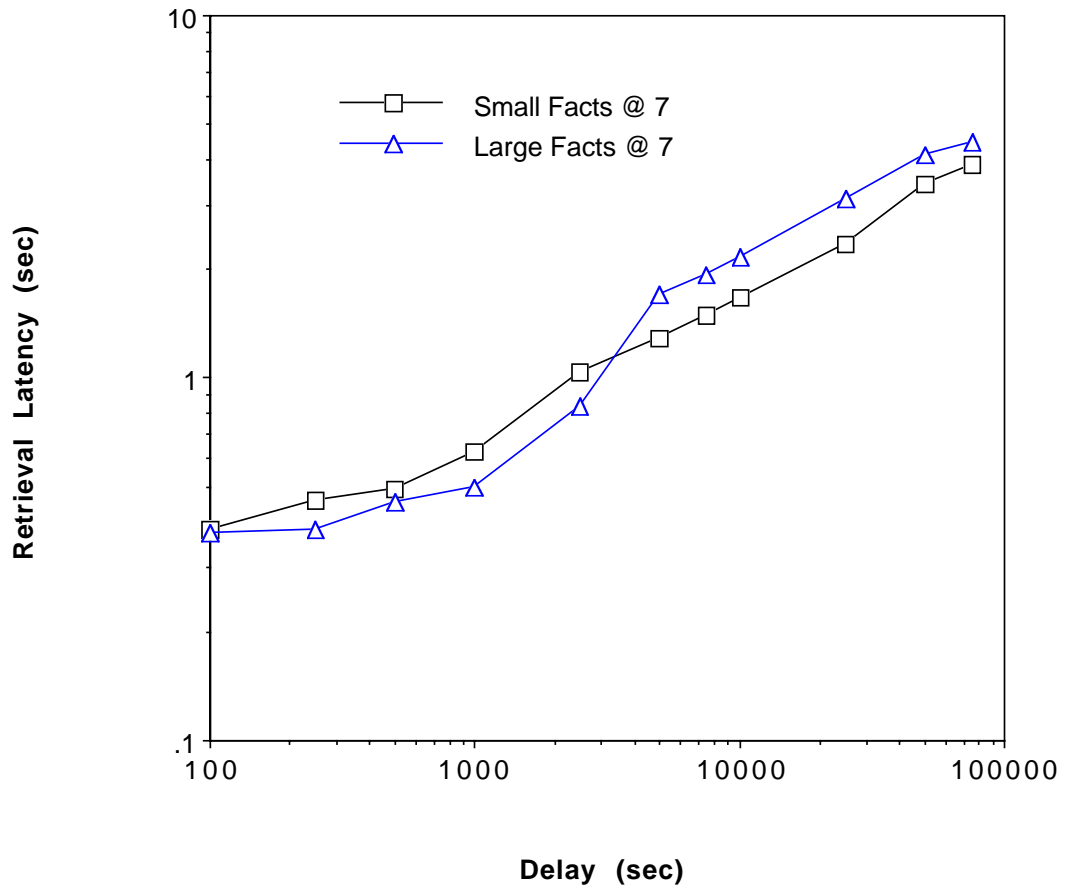

Figure 5.11: Retrieval Latency as a function of Inter-Problem Delay. 
Very short inter-problem delays lead to faster retrievals but also, just as for very low retrieval thresholds, to a pathological inversion of the problem-size effect, as shown in Figure 5.11, which plots the retrieval latency for small and large facts at cycle 7 as a function of inter-problem delay:

In conclusion, the pressure to adopt a more intense presentation schedule is strong, since it leads to much earlier and faster retrievals, but a strong limit is imposed by the catastrophic performance degradation at very short delays ${ }^{23}$. The optimal inter-problem delays of 7500 to 5000 seconds correspond to 4000 to 6000 problems per year respectively, which is consistent with the textbook frequencies reported by Ashcraft \& Christy (1995).

\subsection{Steepness of Frequency Distribution}

Another well-studied characteristic of the domain of cognitive arithmetic that is essential to the present explanation of the problem-size effect is a problem distribution whose frequency decreases with the problem size. As previously mentioned, a linearly decreasing probability distribution was assumed and the slope of the probability density function will be referred to as the steepness of the frequency distribution. That number can be understood as follows: in a 10x10 table, the average fact will have a probability of 0.01. If the slope is 0.0005 (the number used in the simulation), then the probability of neighboring facts in the table will differ by that amount. This implies that the most common fact has a probability of 0.0145 and the least common a probability of 0.0055 , which leads to a frequency ratio between most common and least common facts of about 2.6. Averaging over rows or columns, that also implies that the ratio between most common and least common argument is about 1.6. This corresponds fairly closely to the textbook frequencies reported by Ashcraft \& Christy (1995) and Hamman \& Ashcraft (1986). 
One would assume from the omnipresence of the problem-size effect that the steepness of the frequency distribution would have a significant effect on the performance of the system. Surprisingly enough this is not quite the case, as is shown by Figure 5.12, which plots the odds of retrieval error as a function of the steepness of the frequency distribution for cycles 1, 2, 3, 7 and 20:

Odds of Retrieval Error as a function of the Steepness of the Frequency Distribution

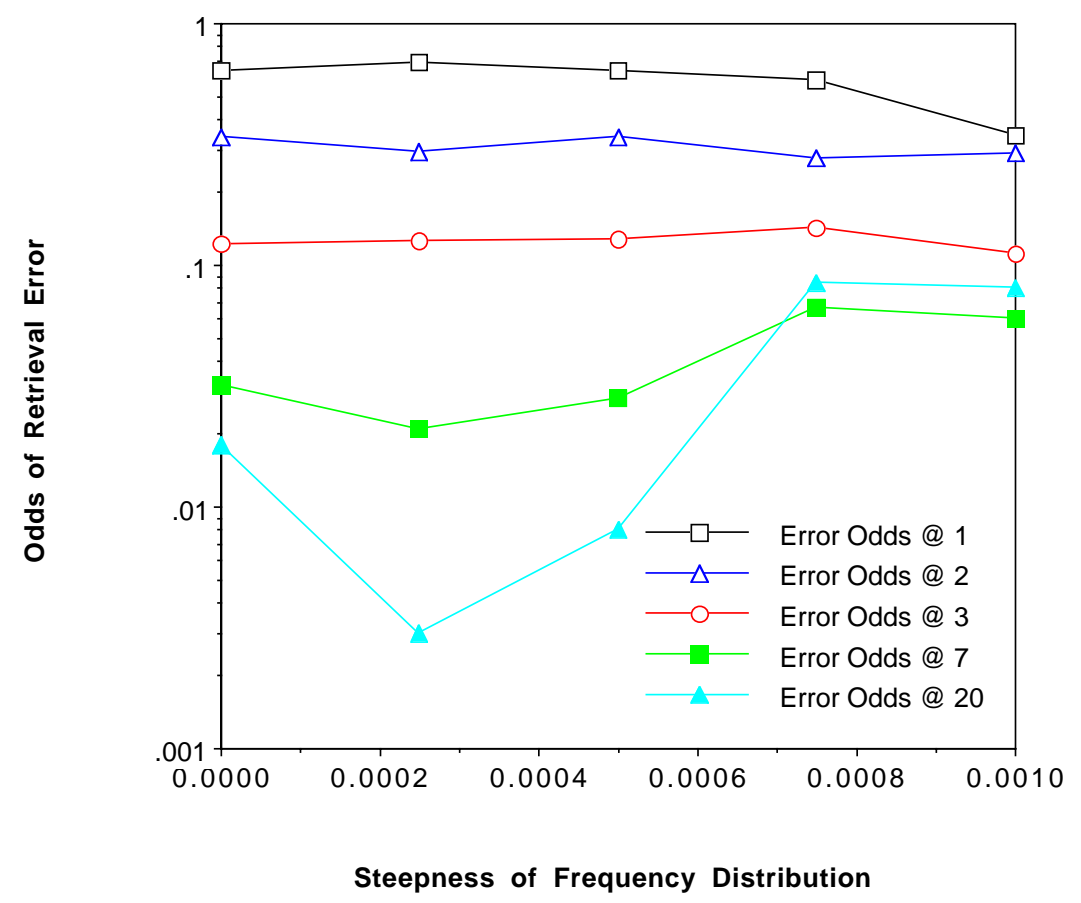

Figure 5.12: Odds of Retrieval Error as a function of Steepness of Frequency Distribution.

During the first three cycles, the odds of retrieval error are fairly insensitive to the steepness of the distribution. However, for very steep distributions (0.00075 and over, which correspond to a ratio between most and least common fact of over 4.0), the odds of error stabilize above 5\% and do not improve further, while they converge to fewer than $1 \%$ for more even distributions of slopes of 0.0005 or smaller. The data for the perfectly even distribution is somewhat unrepresentative, in that a single fact developed a

${ }^{23}$ This of course is somewhat sensitive to other parameters, such as the amount of 
consistent error that could not be improved and which accounted for most of the final error, but it should serve as a reminder of the stochastic nature of the results.

One reason for the absence of a strong effect results from the nature of the distribution itself. The errors caused by a steep distribution will occur for the least frequent problems because they are weakest, but since they are the least frequent problems they will also affect the average error odds the least. Hence the worst of the effect is minimized. But the main reason is that the effect of a regular problem distribution on the relative activation of arithmetic chunks, no matter how steep, is actually quite small compared to the other factors producing variations of activation such as noise, schedule randomness and other non-regular frequency variations. As previously seen, the difference in the base-level activation of two chunks of differing frequencies but equal total lives is 0.5 (i.e. $1-d$ which is usually 0.5 ) times the logarithm of the ratio between the chunk frequencies. Even for the extreme slope of 0.001, which presents the most common problem 19 times more often than the least common one, the average frequency ratio between neighboring facts is 1.1 , which yields a difference in base-level activation of only about 0.025 , which is almost negligible. And because the mismatch penalty sharply increases with distance, the activation differences between neighboring facts are the ones that really matter.

The effect of the steepness of the frequency distribution on odds of retrieval is very limited as well, as Figure 5.13, which plots the odds of retrieval at cycles 1 to 3 as a function of the steepness of the frequency distribution, demonstrates. The curves are by and large flat, with a small effect that changes over time. During the first cycle, when the odds of retrieval are about 0.1 , the steeper distributions have somewhat better retrieval, mainly because their small facts are more frequent than for flatter distributions, and thus have a higher base-level activation and a better chance of being retrieved. During the third cycle, when the odds of retrieval are about 10.0, the flatter distributions have better retrieval, because their large facts are more frequent than for steeper distributions, and thus are more active and have a better chance of being retrieved. The reasoning of cycle

feedback. In general, the optima discussed here are only local. 
1 concerning the small facts is not applicable because those facts are not consistently retrieved independently of the distribution. The second cycle, when the odds of retrieval are about 1.0, combines the advantages of the first and third cycle, with the middle distribution having the lowest retrieval. But all those effects are so small as to be negligible.

Odds of Retrieval as a function of the Steepness of the Frequency Distribution

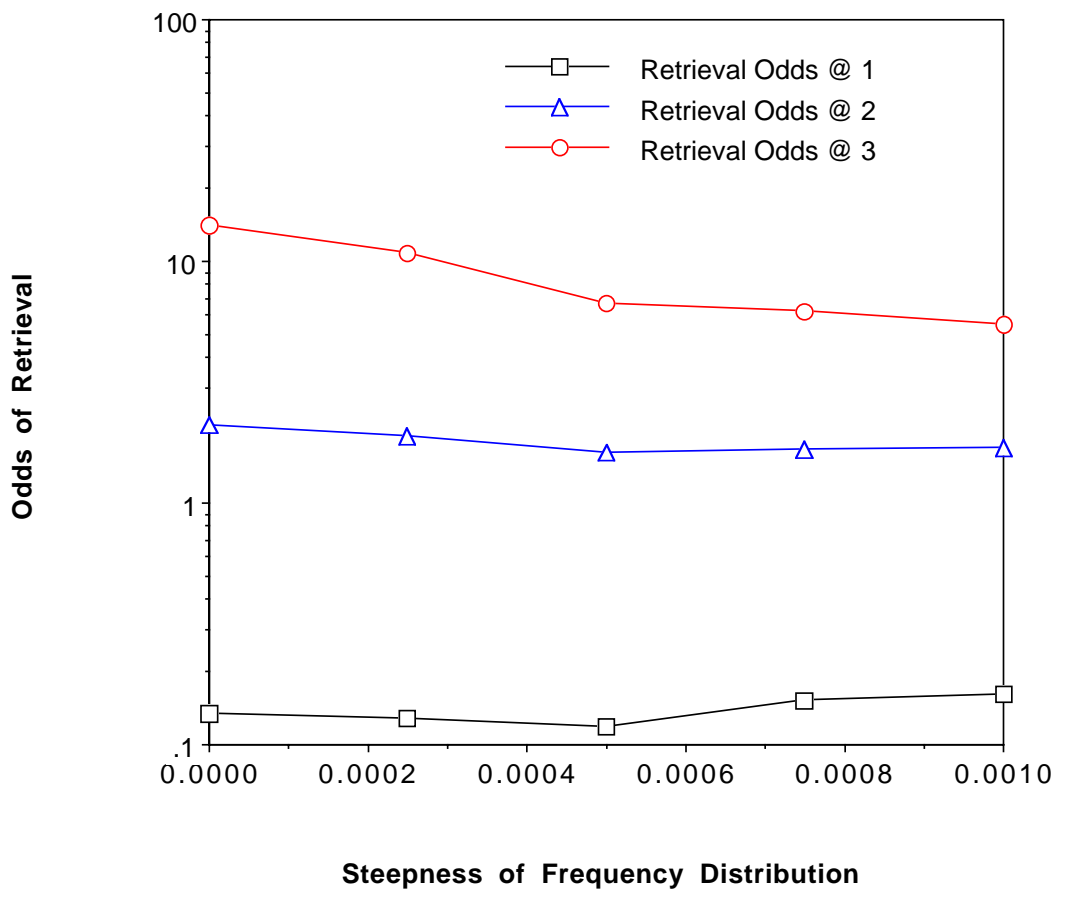

Figure 5.13: Odds of retrieval as a function of Steepness of Frequency Distribution.

The problem-size effect is of course the measure on which the steepness of the frequency distribution has a significant effect. Figure 5.14 plots the retrieval latency for small (single-digit sum) and large (two-digit sum) facts at cycle 7 as a function of the steepness of the frequency distribution. The problem-size effect is 0 for an even distribution and increases about linearly with the steepness of the distribution. One can easily prove through a basic summation argument that the ratio between the sum of the frequencies of small and large facts grows with the square of the steepness of the frequency distribution function, as Figure 5.15 shows. 
Retrieval Latency as a function of the Steepness of the Frequency Distribution

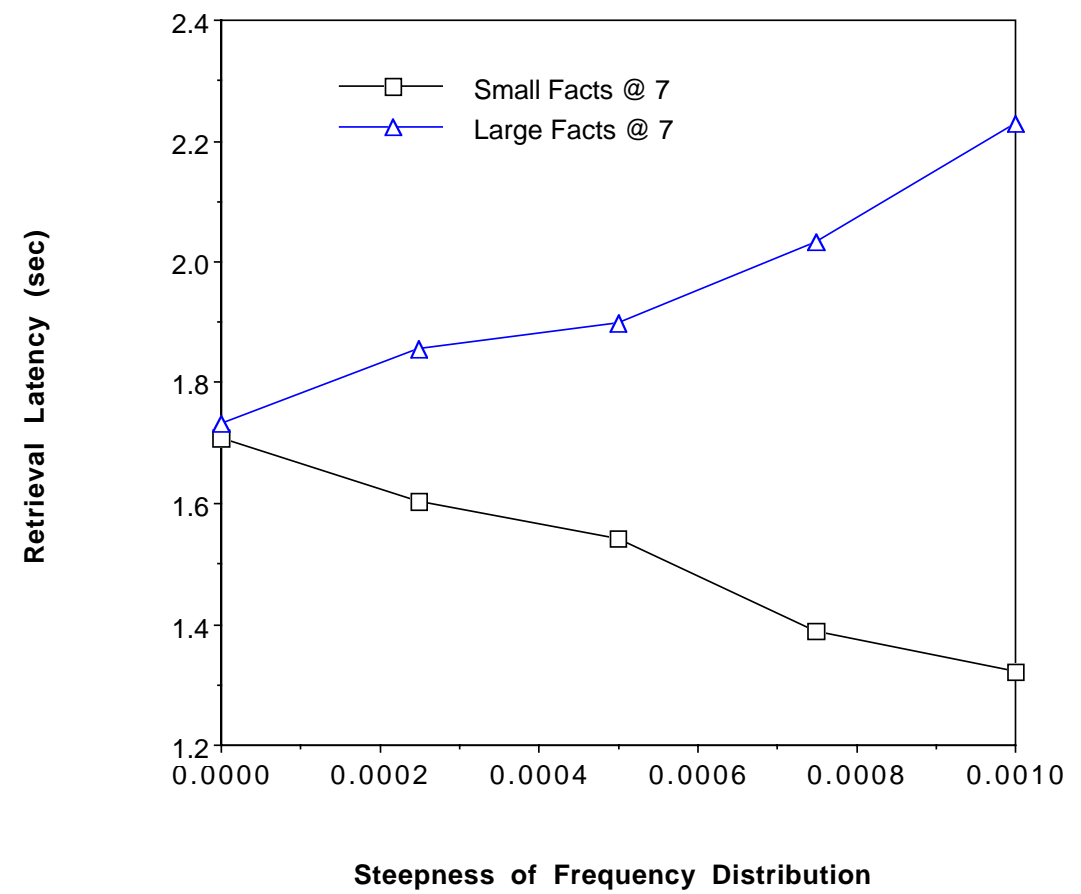

Figure 5.14: Odds of retrieval as a function of Steepness of Frequency Distribution.

Frequency Ratio between Small and Large Facts and Average Latency as a function of Steepness

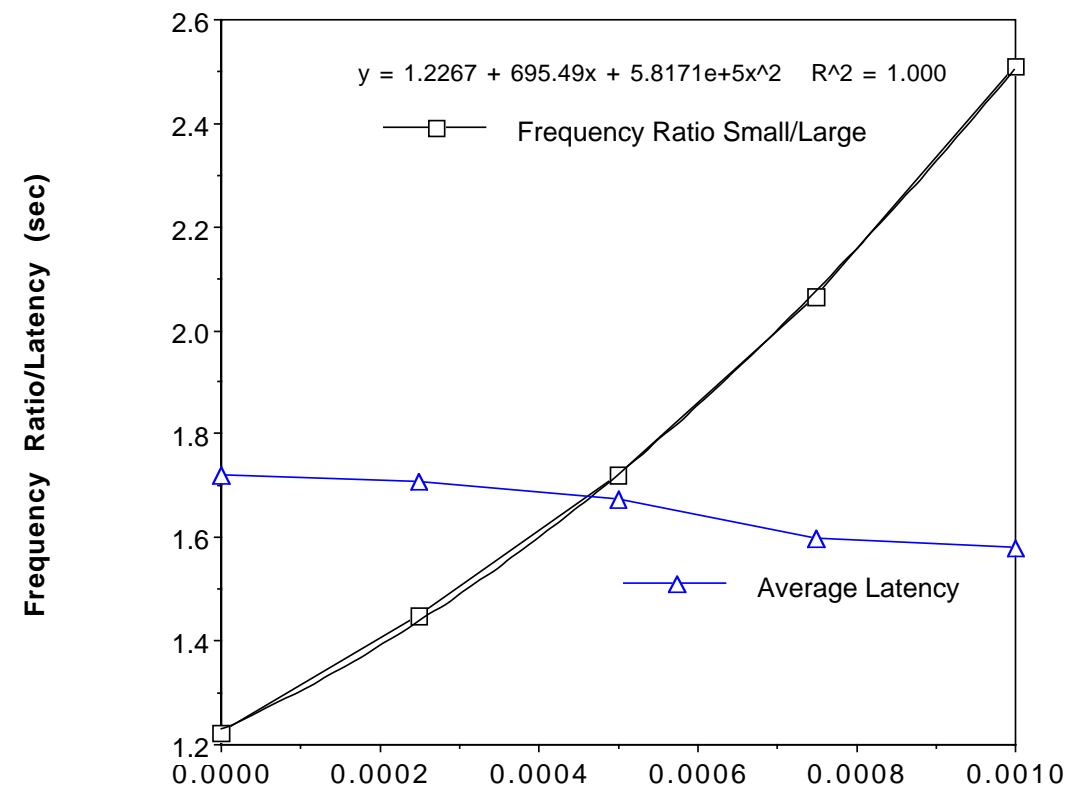

Steepness of Distribution Function

Figure 5.15: Effect of Steepness of Frequency Ratio and Average Latency 
When one expresses the total life of the chunk in terms of the number of references times a constant lag, as was done in the previous section, one can see that the base-level activation grows with (the logarithm of) the frequency of that chunk to the power of $1-d$, which given the default value of 0.5 for the base-level decay exponent is 0.5 . Thus this exponent of 0.5 will cancel the square, and the base-level activation will be proportional to the logarithm of the steepness of the distribution function. Since the retrieval is the exponential of the activation, the problem-size effect will then grow linearly with the steepness of the distribution function.

Of course, even though Figure 5.14 shows that small facts get faster at about the same rate as large facts get slower as the steepness of the distribution increases, since small facts also become more common than large facts the average retrieval latency will therefore decrease with the steepness of the distribution function, as Figure 5.15 shows. This is essentially an information-theoretic argument: the steeper the distribution, the less information it holds and the more predictable it becomes, and through Bayesian adaptation the faster one can perform the task. Since the uneven frequency of presentation of arithmetic facts is at the source of the problem size effect, a common question is whether this frequency differential should be rooted out off textbooks and classroom exercises. This analysis suggests that that need not be the case. Educators should teach arithmetic facts in the same frequency distribution as their students would encounter them in the real world in order to maximize their performance, at least for the range of distribution steepness values for which convergence is assured. In that respect, the steepness value of 0.005 which approximates the empirical textbook distribution and which was adopted in this simulation might well characterize the optimal distribution.

\subsection{Feedback Probability}

Given initial knowledge of the numbers and counting facts, plus operational definition of addition and multiplication in the form of computational procedures, the lifetime simulation could theoretically operate without any further external assistance and gradually recreate and strengthen arithmetic facts until they can be retrieved directly. In 
real life though, children are not left to their own devices but are instead given much formal assistance in the form of repeated and systematic exposure to the correct facts, correction upon error, devices such as calculators, or persons such as teachers or parents who can provide the answer when needed. Of course, modeling this external assistance can become extremely complex. In the lifetime simulation, it is modeled as the probabilistic possibility of requesting the answer when one cannot retrieve it. This probability starts at a value referred to hereafter as the initial feedback probability, and decreases proportionally to the percentage of answers retrieved at the previous cycle to simulate the gradual decrease of supervision over time. If the model cannot retrieve the answer and feedback is not available, then it must use the computation procedures to produce the answer.

The question then is how much feedback is necessary, or more precisely what is the initial feedback probability to ensure convergence to the correct answers? Figure 5.16 plots the odds of retrieval error as a function of initial feedback probability for cycles 3,7 and 20. One can see that initially (at cycle 3, when retrieval becomes the rule) the odds of retrieval error decrease exponentially with the initial feedback probability from odds of about 10.0 for no feedback to odds of about 0.1 for constant feedback. Over time, however, an initial feedback probability of 1.0 will lead to convergence to the correct answers. For a feedback value of 0.9, the odds of error stay around 0.3. For lower values, the odds of error gradually increase over time, to about even odds for a feedback of 0.75 , and much worse for lesser feedback. Thus convergence to the correct answer is very sensitive to the amount of feedback received and requires nearly complete initial feedback. Note however that the feedback probability decreases over time as the model switches to retrieval. Also, the amount of feedback necessary to ensure convergence trades off with other parameters such as the retrieval threshold. For example, a higher retrieval threshold, which would delay the switch to retrieval, would allow a lower feedback probability to still lead to convergence. Finally, remember that the feedback includes explicit instruction and use of devices such as calculators in addition to human feedback. 
Odds of Retrieval Error as a function of Initial Feedback Probability

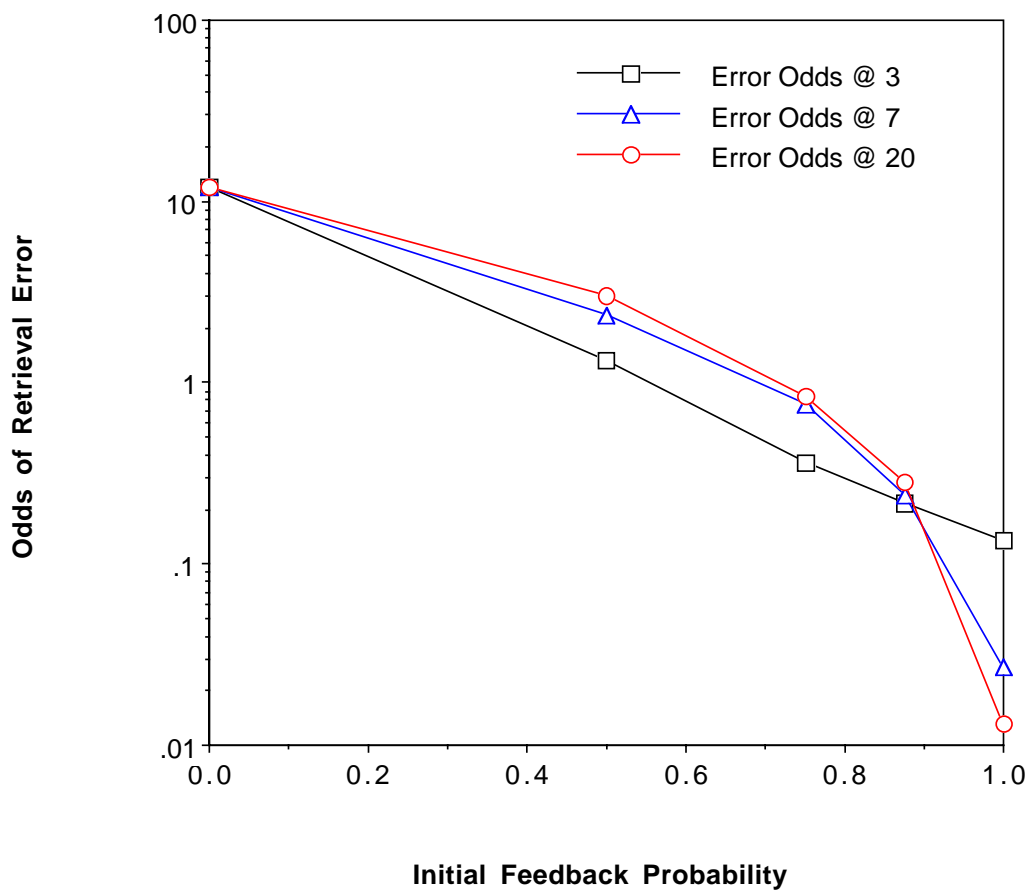

Figure 5.16: Odds of Retrieval Error as a function of Initial Feedback Probability.

Odds of Retrieval Error as a function of Initial Feedback Probability

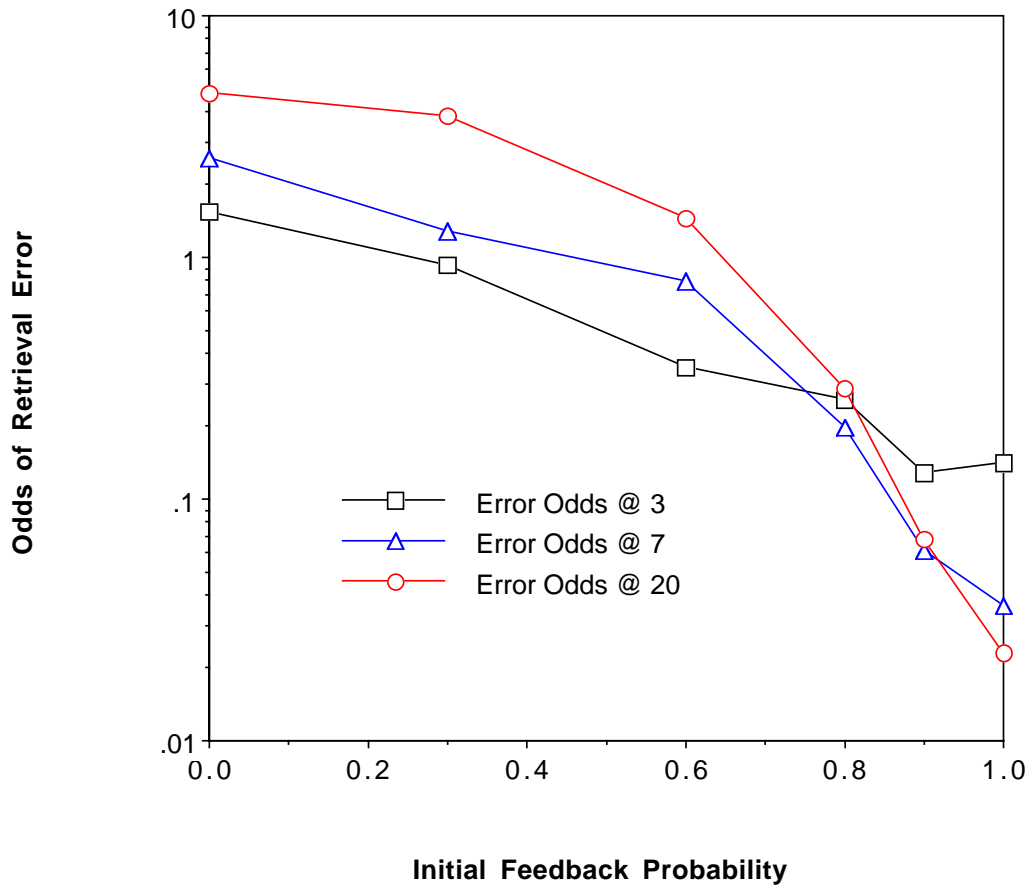

Figure 5.17: Odds of Retrieval Error as a function of Initial Feedback Probability (No *). 
One of the factors disrupting convergence is the uneven statistics of use for addition facts resulting from their use in computing multiplication facts. Figure 5.17 presents the same data as Figure 5.16 for a lifetime simulation of only addition knowledge without the learning of multiplication facts. The previous results are essentially preserved, though the influence of the initial feedback probability is lessened. The odds of error at cycle 3 also decrease exponentially with the feedback, but at a much lower pace. While constant feedback is also needed to a convergence to correct answers, slightly lower levels such as 0.9 stabilize at fairly low levels (e.g. 5\%), but feedback levels at or below 0.6 result in a gradual worsening over time to even odds or worse. Thus even without the disruptive presence of multiplication training a high feedback level remains essential.

One possibility to increase the amount of feedback would be to not only provide it with high probability, but also to amplify it through multiple rehearsals of the correct fact. This technique, however, does not improve performance and can even degrade it. Many additional rehearsals of a particular fact will make it significantly more active than its neighbors, and thus more likely to be incorrectly retrieved when those facts are queried. An error when retrieving those neighboring facts will in turn lead to multiple rehearsals, and so on in a gradually destabilizing arms race between competing facts. Another alternative to improve the feedback would be to immediately correct the answer (if possible) before the goal is popped and becomes or reinforces an erroneous long-term fact. However, as we saw, those erroneous facts are not the primary source of errors because they will be decayed away under any noise value lower than 1.0, and thus very quickly under a noise value such as 0.25 . Over the long term, the main problem comes from the incorrect retrieval of neighboring correct facts. Eliminating these errors depends on developing highly negative strengths of association to those facts from the differing context element. However, a retrieval error results in the automatic strengthening of those associations, and thus cannot be prevented by correcting the goal before it is popped. 


\subsection{Discussion}

The general conclusion is that while the qualitative behavior of the model is preserved across a range of parameter values, specific values for each major parameter of the lifetime simulation can be derived which produce optimal behavior. Excessively aggressive values (i.e. high noise, low threshold or mismatch penalty, fast or uneven schedule, rare feedback) lead to catastrophic performance which gets increasingly worse over time. Values slightly beyond the optimum lead to sub-optimal but ultimately stable performance (e.g. 10\% error). Excessively safe values usually lead to slightly better initial error performance than the optimum but also to an often sharply slower switch to retrieval. Optimal parameter values that maximize performance while ensuring correct convergence exist for each parameter and fit subjects' performance well.

Of course, the analysis in this chapter only establishes that those values are local optima, i.e. that each parameter value is the best with the other parameter values held constant. This does not preclude other optima, which might also provide some better absolute performance than this one in terms of speed and correctness of retrieval. One of these would correspond to a purely symbolic system, i.e. no noise, retrieval threshold or partial matching. A computationally unrealistic search would be needed to catalog them exhaustively. But they would be unlikely to account for the subject data as well as the current simulation parameters. The purely symbolic system is a case in point, offering generally a very poor model of human cognition.

Of course, human cognition evolved in a very different environment than an exact, unchanging domain such as arithmetic. Because they were much less precise and constantly changing, these environments put a much higher premium on the robustly adaptive qualities of stochasticity and approximation that a purely symbolic system lacks. Approximation is essential in a continuous environment. Lebiere \& Wallach (in preparation) show how the ability to generalize to similar problem chunks lead to a flexible and powerful solution to a wide range of control tasks. Lovett (1998) has argued that noise promotes adaptation to a changing environment. West \& Lebiere (in 
preparation) provide an example of the beneficial use of noise without requiring any assumption about the nature of an external environment. The task is the popular game of Paper Scissors Rock (PRS). The ACT-R model memorizes in declarative chunks short sequences (typically of length 2 or 3 ) of the opponent's moves. Then before each move it guesses the opponent's next move by retrieving the most active chunk given the opponent's immediate past move(s). The activation noise in this model serves two purposes. First, it enables the model to reproduce quite well the stochastic nature of human play. More importantly, activation noise increases a model's power by decreasing its predictiveness while still allowing it to exploit the opponent's predictability. Thus given two otherwise identical models with different activation noise levels, the model with the highest noise will over time always win. That noise level is even a much more important performance factor than the length of the move sequences memorized by the model. Since a priori all moves are equally good, what must be learned is not any fixed set of facts but instead the constantly changing patterns of one's opponent. This provides an opposite example to the unchanging certainty of arithmetic knowledge, and is probably more representative of the environment in which the human cognitive architecture has developed.

One way to describe the optimal parameter values arrived at in the previous sections is in term of a speed-accuracy tradeoff, i.e. retrieval is as fast as possible within the constraints of ultimate correctness. One could imagine how the practice of teaching arithmetic evolved to optimize this tradeoff, probably in large part unconsciously. But how could the global parameters, i.e. activation noise, retrieval threshold and mismatch penalty, have been optimized in that way? Those parameters are assumed to be architecturally fixed, and it is doubtful that arithmetic has been part of our culture long enough for evolutionary pressures to have taken hold. It is possible that the base-10 system happened to be optimal in this sense, but that is rather unlikely. All in all, this suggests that human cognition might be even more adaptive than suggested by this model, either through architectural features (e.g. decreasing activation noise) or explicit strategies such as representational recoding. 
The alternative explanation of the correct learning of arithmetic facts through decreasing activation noise as a function of practice was briefly examined in the previous chapter. Essentially, activation noise would decrease as a function of practice, thereby providing variable levels of stochasticity. If one's performance at retrieving certain facts was too random, one could always decrease the noise associated with those facts by increasing their level of practice through rehearsal, i.e. studying. Under this assumption, activation noise is not a fixed quantity that sharply limits the complexity of what can be learned, but instead specifies how much practice is necessary to reach any performance level. Of course, beyond a certain complexity the theoretical amount of practice necessary might not be practically feasible or desirable. A similar reasoning applies to another factor of chunk activation, the mismatch penalty. If the similarity between chunks (e.g. numbers) decreased with their use, then chunks in which they appear as slot values (e.g. arithmetic facts) would become increasingly differentiable and unlikely to be misretrieved. Again, any performance level might be reached given enough practice. This is certainly compatible with the finding that small numbers are better differentiated than large ones (Whalen, 1996). Generally, this suggests a view of architectural parameters such as activation noise and mismatch penalty as specifying a starting point of performance rather than an absolute.

Another way in which cognition could be more adaptive than assumed in this simulation is through the use of explicit strategies. Strategies to reduce noise could include explicit error checking and oversampling. Error-checking strategies include the even-odd rule, as well as specialized rules for example for multiplication by 5 or 9 , and general rules of thumb such as checking the rough magnitude of the answer for compatibility. Oversampling would require performing a retrieval multiple times and checking that the answers are equal to limit the probability of misretrieval. The usefulness of this technique is limited by short-term priming effects (i.e. the misretrieved fact just got rehearsed) and also by the fact that it accounts only for transient sources of noise. Strategies to reduce interference include the massing of problems and representational recoding. Massing of problems (e.g. studying the multiplication by 5 on a particular day) limits the set of highly active facts to a small subset of all arithmetic chunks, and thus 
limits the possibilities for interference ${ }^{24}$. Representational recoding involves changing the declarative representation of facts to facilitate their retrieval. One of the simplest examples would be having separate chunk types for each arithmetic operation instead of having a single arithmetic chunk type. This would essentially represent the operator implicitly in the chunk type instead of explicitly. In addition to eliminating crossoperator confusions, it would remove the operator as a source of activation, leaving the two operands as the only sources, which would limit the odds of error within each type and would lead to faster and more robust convergence. Other examples of representational recoding techniques are discussed in chapters 4 and 6 .

\footnotetext{
${ }^{24}$ This technique would not be effective here since for efficiency purposes the lifetime simulation uses optimized learning which distributes references evenly over the life of the chunk and thus fails to take into account context-specific effects such as massing. On the other hand, it limits errors from precisely that kind of effects such as short-term priming errors.
} 


\section{Chapter 6: Discussion}

\subsection{Feeling of Knowing}

One problem often raised with the model is its assumption that an attempt at retrieval always precedes computation. Reder (Reder, 1982; Reder, 1987; Reder \& Ritter, 1992; Schunn, Reder, Nhouyvanisvong, Richards, \& Stroffolino, 1997) presented evidence that subjects can reliably decide whether to retrieve or compute the answer faster than they can actually retrieve it, a phenomenon referred to as the feeling of knowing. On what basis could subjects make that judgment other than retrieving the fact holding the answer?

Within the framework of this model, one answer lies in a straightforward change of representation. The decision to represent an arithmetic fact as a single ACT-R chunk containing operands, operator and result is a natural choice. It is not, however, the only one. Another is to represent a fact as a chunk that links problem to answer, for example in the case of the addition fact $3+4=7$ :

Fact-3+4=7

isa fact

problem Problem-3+4

result 7

where the problem itself is defined as the chunk linking operands and operator:

\section{Problem-3+4}

isa problem

operand 13

operator +

operand 24

The problem chunk can be seen as resulting from the environmental encoding of the 
question, while the fact chunk results from the solving of the problem. Given a stimulus such as " $3+4$ " on the screen, the model would first construct a representation of the problem, then try to solve the problem, thereby creating the fact chunk. This dichotomy, with one chunk originating from the external environment and the other chunk resulting from internal problem-solving, illustrates nicely ACT-R's theory of the origins of declarative knowledge (Anderson \& Lebiere, 1998). This representation is also consistent with Reder's SAC model (Schunn et al, 1997), where one node represents the problem and points towards another node that contains the solution.

In this variation of the model, solving an arithmetic problem would first require retrieving a chunk representing the problem. If that first step is successful, then the model can infer that it is familiar with the problem and attempt to retrieve the fact holding that problem's answer. Otherwise, it can decide to compute the answer instead. This would provide the model a basis to decide whether to retrieve or compute before it has actually retrieved the answer.

This new representation would also provide a solution to a number of problems. One such problem that was previously discussed was that an operand in a problem would prime a spurious fact that had that number as a result, e.g. the 8 in the problem $1+8$ would prime the result 8 in the fact $1+7=8$. In this alternative representation, the sources $1,+$ and 8 would be used to retrieve the problem $1+8$, then that problem itself would be used as a source of activation to retrieve the fact holding the answer. 8 would not be a source of activation for the latter step, preventing accidental priming of the result slot. This alternative representation would also provide an alternative explanation for a pattern in the addition retrieval data in 4-year-olds, namely the high incidence of errors of type $x+1$ for the addition problem $\mathrm{x}+\mathrm{y}$. In the current representation, counting facts and arithmetic facts are represented as different chunk types, in large part because of their different structures, and thus cannot be mismatched. Under this representation however, all facts would be represented as of the same type, namely the association of a problem to an answer. Since for 4 year-olds counting problems are much more familiar than addition problems, there would be a significant chance of counting problems intruding upon 
addition problems, thereby explaining the $x+1$ pattern of errors (assuming that the problems are fairly similar, or that direct connections from the sources to the facts have been learned). The recoding of tie problems into a distinct representation (e.g. Four Plus Double) is also more plausible if one views the encoding of the problem as an explicit cognitive step, as is the case here.

Finally, this representation would have implications for the ability to use addition (multiplication) facts (e.g. $3+4=7$ ) to perform subtraction (division) problems (e.g. 7 $3=$ ?). The problem is that the components of the subtraction problem ( 3 and 7) are now divided between two chunks, the corresponding addition problem $(3+4)$ and fact (Problem-3+4 =7), which means that associative priming could be difficult. The subtraction answer (4) is in the addition problem, which does not include the addition answer (7), which is given as part of the subtraction problem. One could try retrieving all the addition facts with the given answer, and then test if the corresponding problem includes the proper operand. But such an iterative solution would be costly in time. One possibility would be to develop an associative link from the addition answer (7) to the addition problem $(3+4)$, for example by retrieving the problem when the answer is obtained. Then one can directly retrieve the addition problem without first retrieving the fact since the addition answer will tend to prime the right set of problems. Matching of the problem will only apply to the operand (3) and operator (+), not to the answer (7), but the associative priming from the answer will provide some activation boost ${ }^{25}$. This representation definitely introduces some asymmetry in the access to arithmetic facts, but associative priming in any case had already broken the ideal declarative symmetry.

\subsection{Strategy Choice}

The lifetime simulation assumed that retrieval is always the preferred strategy, and only when it fails (i.e. no chunk can be retrieved) does the model resort to another strategy (computing in this case). As mentioned previously, this is an instance of the Obligatory

\footnotetext{
${ }^{25}$ This underscores the dual roles played by associative activation and partial matching.
} 
Retrieval Assumption of Logan (1988). As discussed in the previous section, it is often assumed that human subjects decide which strategy to use based on the characteristics of the problem, in particular the percentage of success of each strategy. Siegler \& Robinson (1982) and Siegler \& Shrager (1984) show a very strong correlation between percentage of overt strategy ${ }^{26}$ use on each problem and percentage of errors on those problems. The correlation is quite strong between percentage of overt strategy use and percentage of errors on retrieval trials, and still present but much weaker between percentage of overt strategy use and percentage of errors on overt strategy trials. They also report a strong correlation between percentage of overt strategy use on each problem and retrieval latency, and a weaker correlation between overt strategy use and latency of overt strategy.

\section{Correlation between Percentage of Overt Strategy Use and Errors on All Trials}

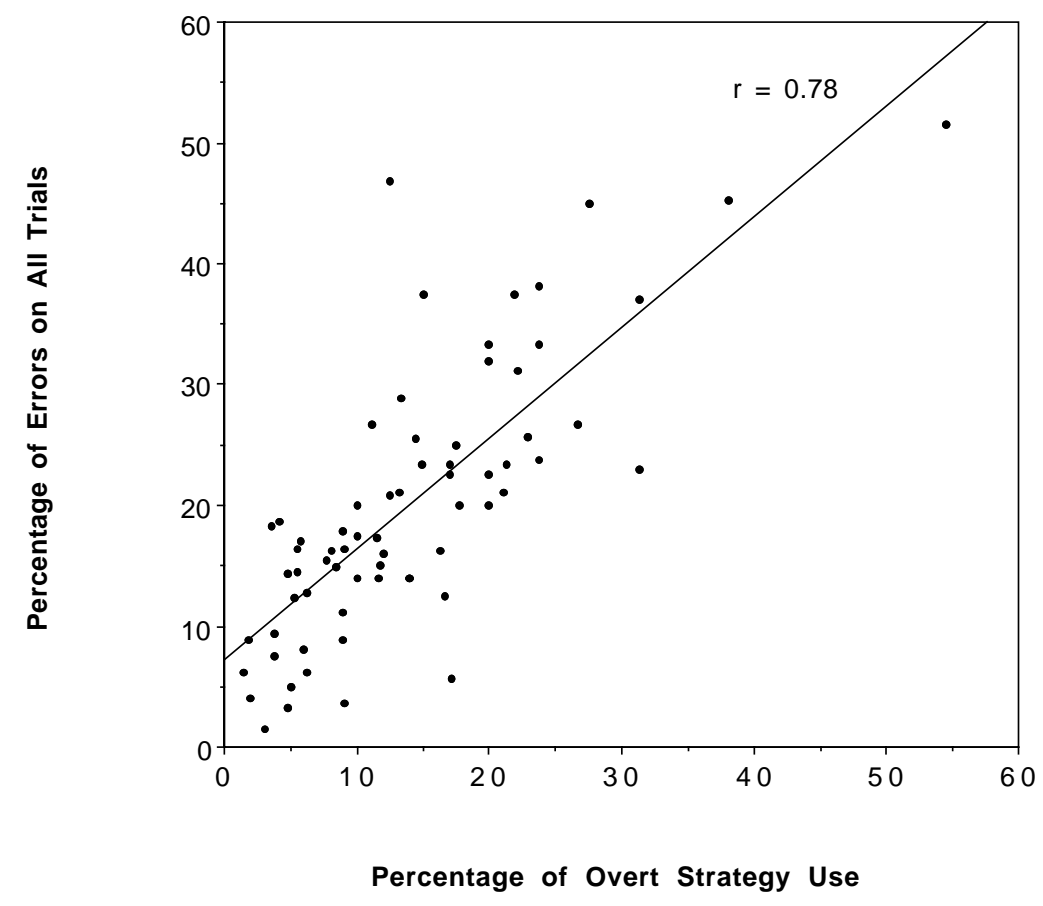

Figure 6.1: Correlation between Percentage of Overt Strategy Use and Errors on All Trials in the Lifetime Simulation.

\footnotetext{
${ }^{26}$ Siegler uses the term "overt strategy" to refer to strategies other than retrieval. In the
} 
To try to reproduce those results, the lifetime simulation was run for one cycle of 2000 problems corresponding to half a year of instruction, to give it some training but still display a significant use of overt strategies. It was then run for 5000 problems without feedback to collect the data displayed below. Figure 6.1 plots the percentage of errors on all trials as a function of the percentage of overt strategy use (computation). The correlation between percentage of overt strategy use and percentage of errors on all trials is quite high (0.78). The correlation between percentage of overt strategy use and percentage of errors on retrieval trials is slightly higher $(0.81)$, whereas the correlation between percentage of overt strategy use and percentage of errors on computation trials is much lower (0.22). Figure 6.2 plots the latency of retrieval and computation trials as a function of the percentage of overt strategy use.

\section{Correlation between Latencies and Percentage of Overt Strategy Use}

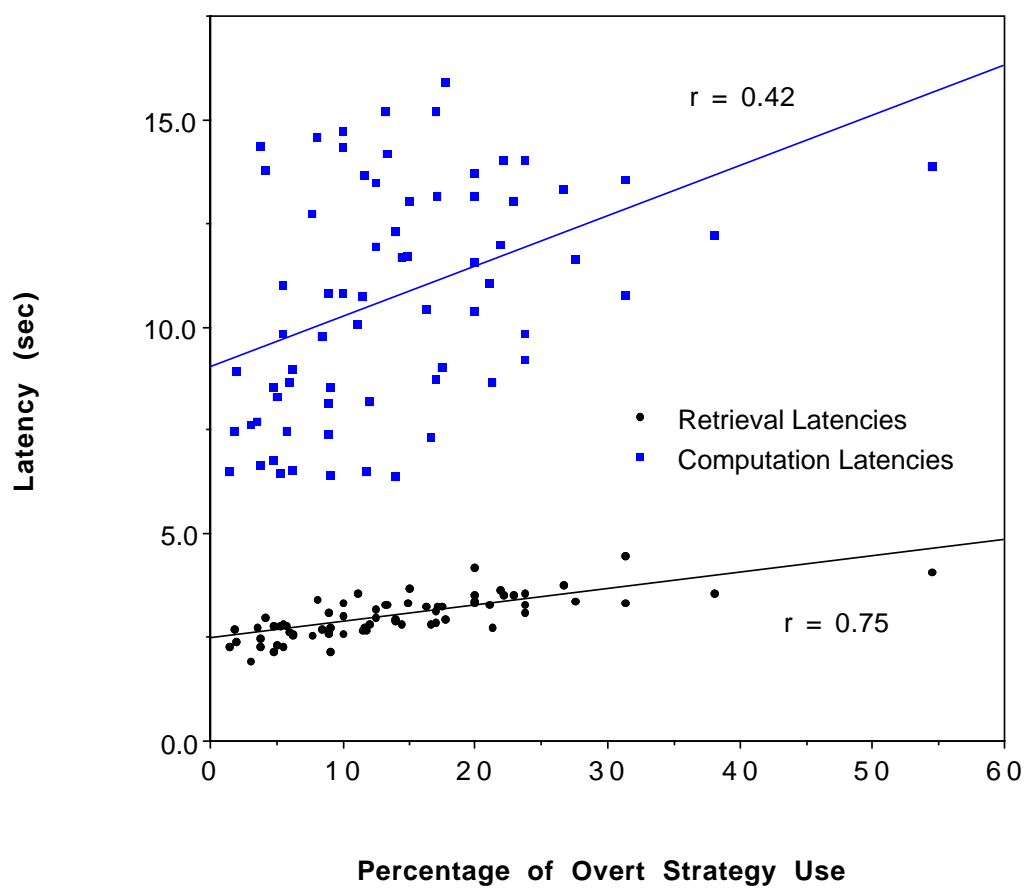

Figure 6.2: Correlation between Percentage of Overt Strategy Use and Latencies of Retrievals and Computations in the Lifetime Simulation.

case of the lifetime simulation, the only overt strategy is computation. 
The correlation between percentage of overt strategy use and retrieval latency is high (0.75), and the correlation between percentage of overt strategy use and computation latency is somewhat lower (0.42). These correlations are quite close to the values in Siegler et al.'s subject data. The lifetime simulation can reproduce those correlations, even though it does not perform any explicit choice of strategy. The reason is straightforward. Since performance improves with practice, a very active fact will be retrieved often, because retrieval is always the first strategy attempted and a very active chunk is highly likely to be above threshold. A very active fact will also be retrieved reliably, because it has seen a lot of practice, which has reduced the odds of retrieval error from other chunks. And finally, a very active fact will be retrieved quickly, because of the direct relationship between activation and retrieval latency. Conversely, weak chunks are less likely to be retrieved and will often necessitate the use of some overt strategy, but are also more likely to see interference from stronger chunks when retrieval is attempted. They will also be slower to retrieve. Thus percentage of overt strategy use is highly correlated with both percentage of errors and latency of retrieval trials. The correlation between percentage of overt strategy use and percentage of errors and latency of those trials is more indirect. Larger problems are more likely to require the use an overt strategy, but are also more likely to result in an error because the computation required often increases with size. Of course, that is not always the case, i.e. $9+1$ is easier to compute than the smaller $3+5$, leading to the more limited correlation. Similarly, larger problems tend to take longer to compute but not always because the number of iterations only depends on one argument.

In conclusion, errors do not determine strategy choice but merely tap into the same underlying variables, namely activation strength and problem complexity. Of course, this does not preclude a more elaborate model, in which the choice of a strategy would be made depending upon the characteristics of the problem and the strategy's past history of success, as Siegler \& Shipley (1995) propose. However, the evaluation of production utility in ACT-R 4.0 is sensitive to the production's overall past history of success but, unlike earlier versions of ACT-R, it cannot be sensitive to the particular problem characteristics. But a declarative record could be kept of past instances of problems and 
strategies adopted and a decision on which strategy to adopt could always be made explicitly by matching the current situation to past records, rather than based on the implicit utility of the productions implementing each strategy.

\subsection{Multiplication Errors}

The previous chapters examined the patterns of errors of addition retrieval. The patterns of errors for multiplication are quite rich, but harder to examine systematically because they take place over a wider range of values and display some characteristics (table errors, close misses, etc) which are difficult to average over and plot together. For those reasons, let us concentrate on the pattern of errors for a single problem. Siegler (1988) reports the answers to multiplication problems for an experiment in which third- and fourth-graders were instructed to state the answer to the problem without resorting to any explicit strategies. Figure 6.3 plots the percentage of answers to the problem $6 \times 9$.

Data for Problem 6x9 from Siegler (1988)

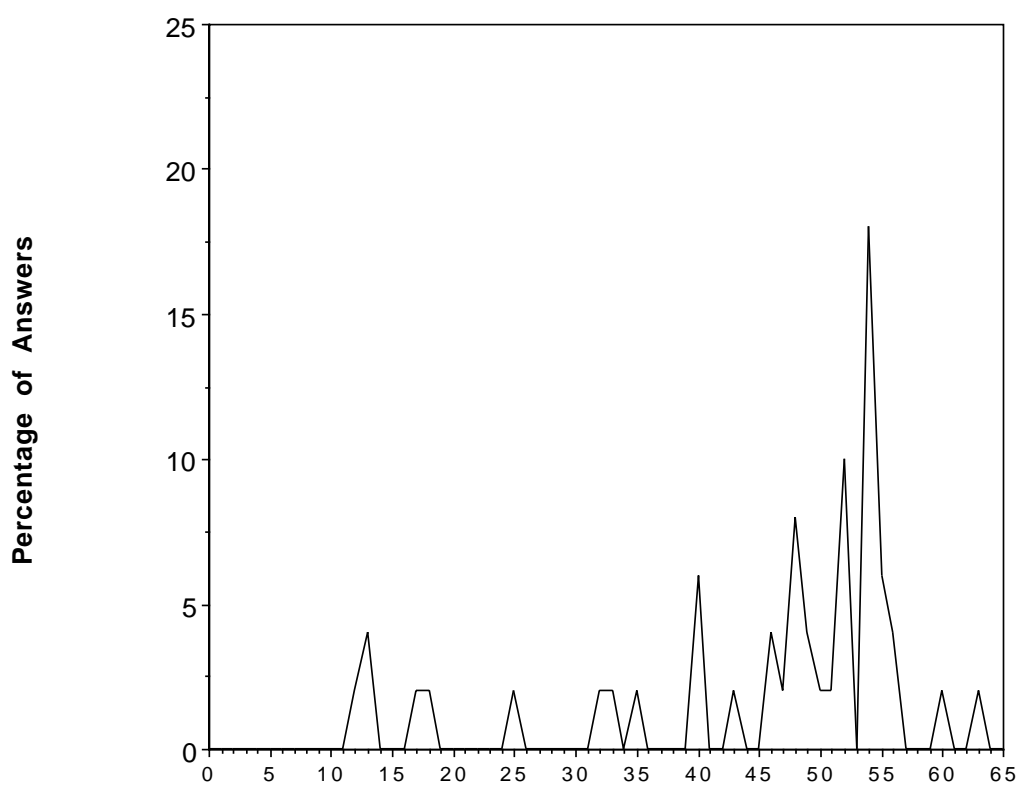

Answers

Figure 6.3: Percentage of Retrieval Answers to the Problem $6 \times 9$ by $3^{\text {rd }}$ and $4^{\text {th }}$ Graders. 
The correct answer, 54, is also the most likely one but only constitutes fewer than $20 \%$ of all answers. The data is fairly noisy, because it corresponds to a single data point for fewer than 50 children, but some error patterns are clearly present. As in the case of addition errors, most of the errors are smaller than the correct answer, with the percentage of answers generally decreasing with the distance from the correct answer. Some of the errors can be classified as table errors, i.e. the answers appear in the same row or column of the multiplication table as the correct answer, e.g. 48=6x8. But most errors are not table errors, either because they are answers to facts elsewhere in the multiplication table (e.g. $5 \times 8=40$ is neither on the same row or column as $6 \times 9$ ) or because they do not appear as answers in the single-digit multiplication table at all (e.g. 46 or 52).

To try and replicate those results, the lifetime simulation was run for 4 addition and 2 multiplication cycles. This corresponds to the end of third grade, considered a mid-point between the approximately even mix of third- and fourth-graders in the subject population. The percentage of answers could have been estimated using repeated sampling, but to limit the amount of variation it was directly computed using the Chunk Choice Equation. Figure 6.4 plots the percentage of retrieval answers to the problem $6 \times 9$. One positive result is that the percentage of correct answers corresponds closely to the data. Most of the errors are also smaller than the correct answer and the percentage of errors tend to decrease with the distance from the correct answer. This is to be expected since smaller facts are more active than larger ones, and the mismatch penalty increases with the distance from the correct fact. Unlike the data, almost all the errors are table errors, with $56=7 \times 8$ being one of the rare exceptions. The most common error is 36 , in large part because it appears as an answer in both the same row $(6 \times 6=36)$ and column $(4 \times 9=36)$ as the correct fact. This preponderance of table errors is not unexpected. The activation penalty imposed for mismatching an argument is relatively small compared to the loss in activation from the absence of the argument as a source, and often the negative association between the mismatching value and the chunk. Therefore, a chunk which mismatches badly on one argument (e.g. 6x5) is more likely to be retrieved than one which mismatches slightly on both arguments (e.g. $5 \times 8$ ), because even though the sum of the mismatch penalties is smaller for the latter chunk, it pays the price in associative 
activation not once but twice, and that is the decisive factor.

Percentage of Retrievals for $6 \times 9$

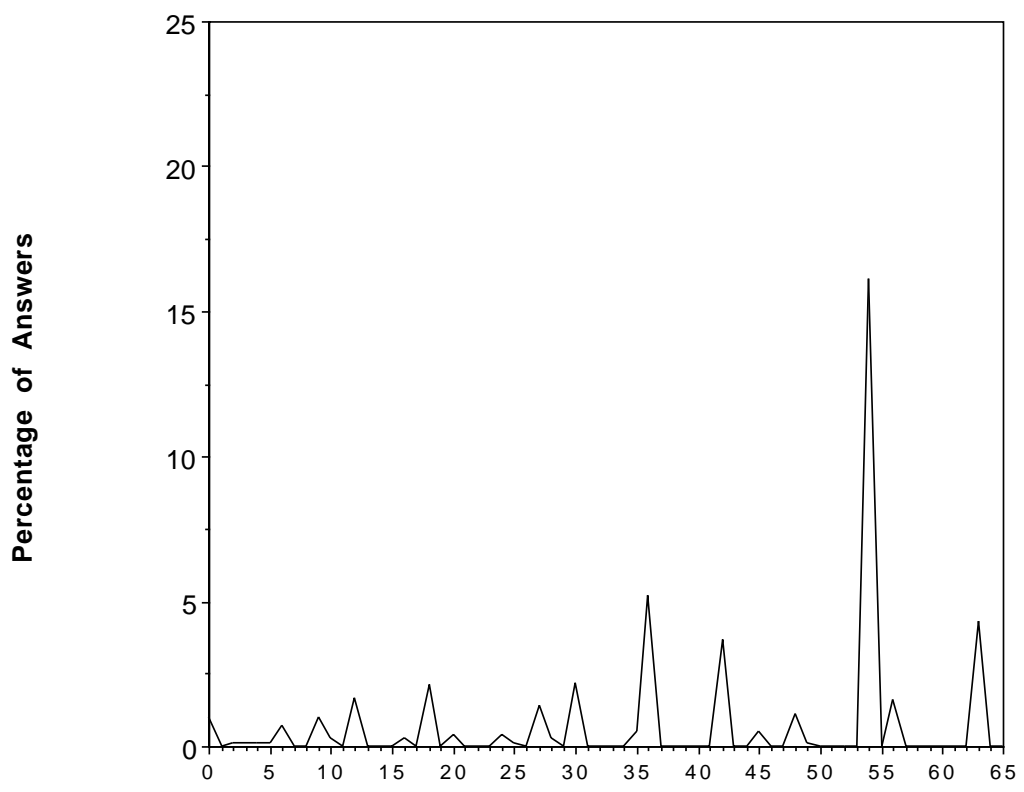

Answers

Figure 6.4: Percentage of Retrieval Answers to the Problem 6x9 by the Lifetime Simulation at the End of the $3^{\text {rd }}$ Grade.

There are also few close misses. Finally, about half the time no fact would reach the retrieval threshold and the model would fail to answer. Of course, subjects too have poorly established multiplication facts at that point. In a related experiment, Siegler (1988) reports that a similar group of children used retrieval to solve single-digit multiplication problems only $68 \%$ of the time, choosing the rest of the time to perform repeated addition, write the problem or count sets of objects. Since $6 \times 9$ is one of the largest multiplication problems, one would expect the probability of using retrieval on that problem to be even lower than the average, which is compatible with the lifetime simulation results reported above. But since subjects in the other experiment were instructed to state what they thought was the right answer without resorting to backup strategies, the question is how they produced the answer if they could not rely on retrieval. One possibility is that they were estimating, i.e. guessing, what the correct answer was. This would explain the wide range of errors and the low percentage of table 
errors. But how could an ACT-R model guess an answer to a problem such as 6x9? It could generate a number randomly, but one would not expect to find the pattern of close misses and decreasing frequency with distance from the correct answer that was observed in the data. It could repeatedly sample for the answer, or even free-associate to the activation sources $6, x$, and 9 without trying to match them to a particular pattern and thus rely solely on activation to produce the answer. But again one would expect a preponderance of table errors because the most likely multiplication facts to be retrieved would involve 6 and/or 9.

But it seems that when humans engage in this sort of estimation, they do not do so randomly but rather compensate the lack of a specific fact by relying on a wider base of related facts. For example, they might answer 46 or 52 to $6 \times 9$ not because they have retrieved a specific fact with that answer but because similar problems have similar answers. And they might do so not by explicitly sampling those facts iteratively and then averaging their answers, which they were not given the time to do, but by relying on a more fundamental sense of numbers and arithmetic facts. How could that reliance on a set of facts rather than a single one be implemented in ACT-R? Such a mechanism has been proposed by Lebiere \& Wallach (in preparation) to perform a similar task, interpolation, in a number of control problems. Interpolation, like estimation, relies on a number of facts but, rather than selecting a specific one which is by definition unavailable, it must find the best compromise between them. The solution is to produce the answer that minimizes the mismatches between that answer and the answers from each specific fact, weighted by its probability of retrieval. Formally, the answer is the value $\mathrm{V}$ that minimizes the following quantity:

$$
V=\operatorname{Min} \sum_{i} P(i) \cdot\left(1-\operatorname{Sim}\left(V, V_{i}\right)\right)^{2} \quad \text { Estimation Equation }
$$

where $P(i)$ is the probability of retrieving chunk $i$, as determined by the Chunk Choice Equation, $V_{i}$ is the value specified by chunk $i$, and the term in parenthesis is the dissimilarity between the values, i.e. the amount of mismatch between them. If the 
dissimilarity between the values is interpreted as the error, then the Estimation Equation can be viewed as a standard least-squared error method. The well-known result that least-squared error solutions can be shown under certain assumptions to correspond to maximum likelihood hypothesis (e.g. Mitchell, 1997) provides the connection between this equation and ACT-R's Bayesian framework.

This mechanism is applied to the current task in the following way: after the lifetime simulation is run for the equivalent of three grades, the problem $6 \times 9$ is repeatedly submitted 50 times (roughly corresponding to the number of subjects). If a fact reaches the activation threshold, then it is retrieved and that answer is given. If no fact reaches the threshold, then the probability of retrieving each chunk (ignoring the activation threshold) is computed using the Chunk Choice Equation and the Estimation Equation is computed for all integer values. The number that minimizes that equation is given as the answer.

Percentage of Retrievals and Guesses for $6 \times 9$

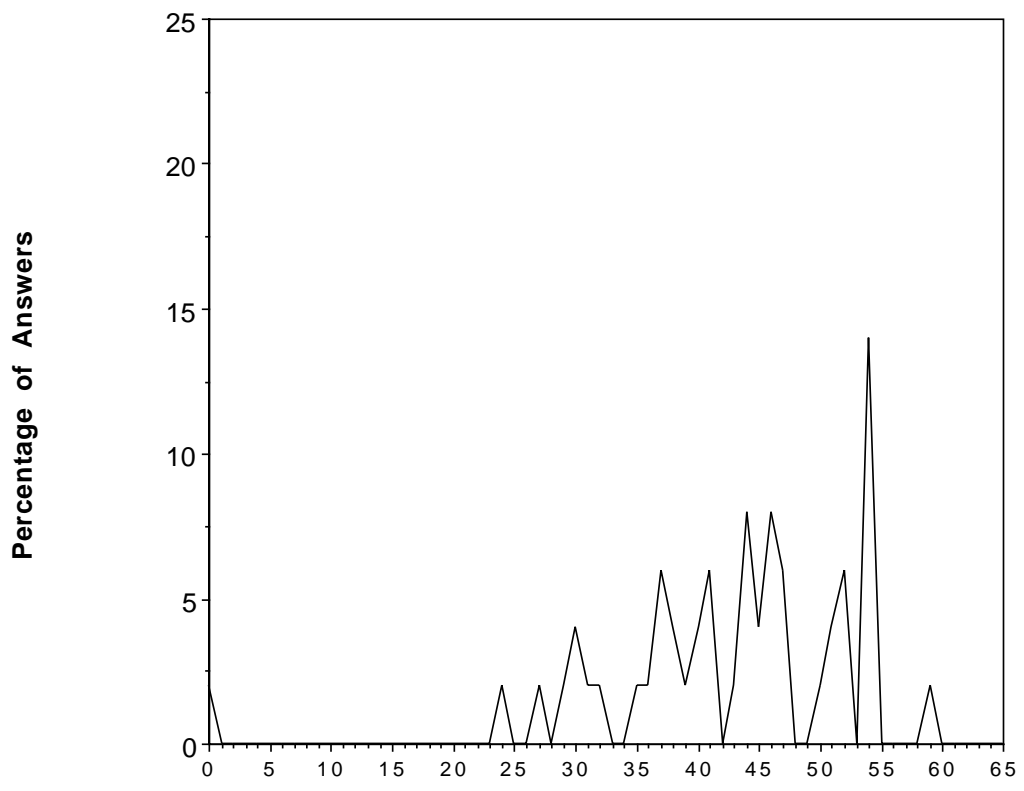

Answers

Figure 6.5: Percentage of Retrieval and Guessing Answers to the Problem 6x9 by the Lifetime Simulation at the End of the $3^{\text {rd }}$ Grade. 
Figure 6.5 plots the percentage of combined retrieval and guessing answers for the problem 6x9. Of course, this is just a sample run, and as previously mentioned the amount of variation between runs is high. Therefore, it does not reproduce each individual answer percentage, but it displays the right characteristics. The proportion of correct answers is about right, which results from the standard retrievals as shown in Figure 6.4. Most of the errors are smaller than the correct answer, because smaller facts are more active, and thus have a higher probability of being retrieved and thus weigh more in the Estimation Equation. Most of the errors are not table errors, because estimation is a continuous process in which the answers to actual facts have little advantage over neighboring numbers. And there are many near-misses, with the probability of error decreasing with the distance to the correct answer. This results from the fact that while smaller facts are generally more active, facts closer to the current answer will have a better match score because they will incur a smaller mismatch penalty.

The Estimation Equation is similar to an algorithm known in machine learning as the Bayes optimal classifier (Mitchell, 1997). Instead of applying the Maximum Likelihood or Maximum A Posteriori hypothesis to categorize a new instance, the Bayes optimal classifier weighs all the hypotheses according to their posterior probabilities, and combines them to produce the most probable classification. As its name implies, this algorithm cannot be outperformed by any other classification algorithm (using the same hypothesis space and prior knowledge). While it is generally considered too computationally expensive to be of direct practical use, its implementation is ACT-R is not prohibitively slow, and its behavior seems to correspond closely to the human ability to operate gracefully in continuous environments. And of course, as Mitchell (1997) points out, more practical algorithms can often be found that asymptotically approximate the characteristics of less feasible but optimal standards such as the Bayes optimal classifier. 


\section{Chapter 7: Conclusion}

\subsection{Difficulties}

ACT-R has matured sufficiently as both a cognitive theory and a software system that it is now possible to target a non-trivial task or result, write a model for it, collect its predictions, optimize its parameters and obtain a very good fit to the data in less than a day. This is indeed the modus operandi of the ACT-R summer school, in which the daily assignment is to model an actual data set, and has been replicated in actual research numerous times. Needless to say, the development of the lifetime simulation took considerably longer. Of course, gratification is not always immediate. Some models of complex tasks such as scientific discovery (Schunn \& Anderson, 1998) are themselves complex, requiring dozens of chunks types and hundreds of productions that take much longer to engineer. But this is clearly not the case here, and it is worth examining how and why this endeavor stretched the limits of the architecture. There were a number of factors detailed below, which were not independent but rather combined to greatly lengthen the total development time.

An obvious limit is computational. While running the simulation didn't actually take a lifetime, and in fact was at least an order of magnitude faster than humans subjects, it lasted between an hour (i.e. lunch) and 15 hours (i.e. overnight) depending on the version. This clearly puts a damper on the development cycle.

Another factor is the statistical and dynamical nature of the learning taking place in the simulation. If modeling had consisted in engineering chunks and productions to produce a specific behavior, the debugging process would have been relatively straightforward. But this wasn't the case; stochastic and error-prone behavior is not accidental but actually essential in modeling this domain. And since the model learns not (only) from a specific, fixed environment but mainly from its own operation, the difficulty lies in detecting when 
the pattern of stochasticity and errors deviates from the norm, and what the statistical causes within the model might be.

Another difficulty is the global character of associative learning. Unlike base-level learning, in which the base level of a chunk is affected only by the references to that particular chunk, associative learning is a more global form of learning. The strength of activation between a source and a chunk will be affected not only by the retrievals of that chunk when that source is active, but also by the retrievals of the chunk in the context of other sources, by the retrievals of other chunks within the context of that source, and indeed by any retrieval at all. This severely limits the ability to develop the model in a modular fashion. For example, one can develop a very precise model of addition, only to see it substantially change when multiplication is introduced. While some effect is to be expected, the extent of the global disruption resulting from associative learning is clearly undesirable.

Finally, an obvious source of difficulties was the fundamental problems with associative learning itself. Often, the learning mechanisms will perform as expected and produce the desired results automatically. This seemed to be the case when the theoretical analysis predicted, somewhat surprisingly, that associative learning would lead to a gradual reduction in errors. While that analysis was generally empirically confirmed, associative learning also had many unintended and undesirable consequences that were not forecast. Much of the development consisted in finding ways to work around those drawbacks, which was difficult considering the ubiquity of the associative learning mechanism.

\subsection{Contributions}

This ACT-R model of cognitive arithmetic offers a number of contributions to the field of cognitive arithmetic, to the ACT-R architecture itself, and more generally to the field of cognition and machine learning.

1. It provides a precise account of a number of central results in the field of cognitive 
arithmetic. While the model is broadly consistent with previous activation-spreading theories of cognitive arithmetic, its basis in a general-purpose Bayesian learning architecture provides a systematic account of the causes and conditions of these effects.

2. It provides a number of practical lessons for the teaching of arithmetic. Because the model makes detailed predictions that are affected by every aspect of the simulation, it can predict which conditions are critical to learning (feedback, spacing) and which are not (regular frequency differences).

3. It contains a number of lessons for the architecture, including the view of retrieval as subgoaling to limit the sources of activation to those critical context elements, and especially exposes the deficiencies of the assumptions behind the associative learning mechanism, and suggests ways to correct them. The nature of the task, requiring a fairly simple model but a very long, mostly self-correcting, learning simulation, was essential in deriving these lessons.

4. From a machine learning perspective, it suggests that despite its past success, a popular algorithm such as the naïve Bayes classifier has limitations in modeling the full scale of human cognition. It also suggests that despite being viewed as too computationally expensive, a more powerful algorithm such as the Bayes optimal classifier in fact closely characterizes some of the aspects of human performance, which suggests that a suitable approximation has been implemented efficiently in the brain.

5. It includes a study of the sensitivity of the model to parameter values, which confirms that the model's qualitative predictions hold for a range of values. However, the analysis also establishes that the values of those parameters, both architectural and domain-specific, that are used in the lifetime simulation are in fact optimal for some measure of performance. Since the human cognitive system was presumably not developed to perform precise tasks such as arithmetic, this raises further questions about the actual extent of its adaptiveness.

6. It advances a view of cognition as a dynamic system (e.g. van Gelder, 1998). Unlike fixed models or models that learn from an external environment, the behavior of this model and its changes over time are primarily determined by its own operations, 
which follow internal dynamics that depend upon the fundamental parameters of the architecture. Because of the richness and variety of those non-linear dynamics, those models are better able to explain the full diversity of human cognition. 


\section{Tables}

\section{Table 1: Equations}

Activation 6

$\begin{array}{lr}\text { Base-Level Learning } & 7\end{array}$

$\begin{array}{ll}\text { Optimized Learning } & 7\end{array}$

$\begin{array}{ll}\text { Posterior Strength } & 7\end{array}$

$\begin{array}{lr}\text { Prior Strength } & 8\end{array}$

$\begin{array}{lr}\text { Empirical Ratio } & 8\end{array}$

$\begin{array}{lr}\text { Match } & 8\end{array}$

$\begin{array}{lr}\text { Retrieval Probability } & 9\end{array}$

$\begin{array}{ll}\text { Chunk Choice } & 9\end{array}$

$\begin{array}{ll}\text { Retrieval Time } & 9\end{array}$

$\begin{array}{ll}\text { Expected Gain } & 10\end{array}$

Dynamic Odds 36

$\begin{array}{ll}\text { Rehearsal Ratio } & 37\end{array}$

$\begin{array}{ll}\text { Retrieval Odds } & 37\end{array}$

Noise Reduction

$\begin{array}{ll}\text { Estimation } & 110\end{array}$ 


\section{Table 2: Symbols}

$A_{i} \quad$ activation of chunk $i$ in Activation and Match Equation

assoc weighting of prior strength in Posterior Strength Equation

$B_{I} \quad$ base-level activation of chunk $i$ in Activation, Base-Level Learning and Optimized Learning Equation

c scaling constant in Rehearsal Ratio and Retrieval Odds Equation

$C$ estimated cost of achieving the goal using the production in Expected Gain Equation

$d \quad$ decay rate in Base-Level Learning and Optimized Learning Equation

$E \quad$ expected gain of production in Expected Gain Equation

$E_{j i} \quad$ empirical ratio in Posterior Strength and Empirical Ratio Equation

$f \quad$ latency exponent (usually at its default value of 1) in Retrieval Time Equation

$F \quad$ total frequency of past opportunities (productions matched) in Empirical Ration

Equation; Also latency scale factor in Retrieval Time Equation

$F\left(C_{j}\right)$ frequency of source $j$ being in the context in Posterior Strength and Empirical Ratio Equation

$F\left(N_{i}\right) \quad$ frequency of chunk $i$ being needed (retrieved) in Empirical Ratio Equation

$F\left(N_{i} \& C_{j}\right) \quad$ frequency of chunk $i$ being needed (retrieved) when source $j$ is in the context in Empirical Ration Equation

$G \quad$ value of the goal in Expected Gain Equation

$L \quad$ life of chunk $i$, i.e. time since its creation, in Optimized Learning Equation

$m \quad$ total number of chunks in memory in Prior Strength Equation

$M_{i p} \quad$ match score of chunk $i$ to production $p$ in Match, Retrieval Probability, Chunk Choice and Retrieval Time Equation

MP mismatch penalty scaling constant in Match Equation

$n \quad$ number of past references in Base-Level Learning, Optimized Learning and Noise Reduction Equation; Also fan of the source of activation in Prior Strength Equation; Also amount of practice in Rehearsal Ratio and Retrieval Odds Equation

$O d d s_{1}$ odds of retrieving chunk $C_{l}$ in Dynamics Odds and Retrieval Odds Equation 
$P \quad$ probability that a chunk can be retrieved in Retrieval Probability Equation; Also estimated probability of achieving the goal in Expected Gain Equation

$P(i) \quad$ probability of retrieving chunk $i$ in Chunk Choice and Estimation Equation

Ratio $_{1}$ ratio of the past frequencies of rehearsal of chunks $C_{1}$ and $C_{2}$ in Dynamics Odds and Rehearsal Ratio Equation

$R_{j i}{ }^{*} \quad$ exponential of prior strength of association in Posterior Strength and Prior Strength Equation

$\tau \quad$ standard deviation of activation noise in Retrieval Probability Equation

$s \quad$ measure of activation noise equal to $\sqrt{ } 3 \sigma / \pi$ in Retrieval Probability Equation

$S \quad$ initial activation noise level in Noise Reduction Equation

$S_{n} \quad$ activation noise level as a function of practice in Noise Reduction Equation

$\operatorname{Sim}(v, d) \quad$ similarity between desired slot value $d$ and actual slot value $v$ in Match and Estimation Equation

$S_{j i} \quad$ strength of association from activation source $j$ to chunk $i$ in Activation and Posterior Strength Equation

$S_{p} \quad$ strength of production $p$ in Retrieval Time Equation

$\tau \quad$ retrieval threshold in Retrieval Probability Equation

$t$ temperature as a function of activation noise $t=\sqrt{ } 2 \mathrm{~s}$ in Chunk Choice Equation

Time $_{i p}$ time to retrieve chunk $i$ in production $p$ in Retrieval Time Equation

$t_{j} \quad$ time elapsed since $j$ th reference in Base-Level Learning Equation

$V \quad$ value that minimizes the squared sum of dissimilarities in Estimation Equation

$V_{I} \quad$ value returned by chunk $i$ in Estimation Equation

$W \quad$ total amount of source activation, usually set at 1 and divided evenly among sources

$W_{j} \quad$ weighing of the activation source $j$ in Activation Equation 


\section{Table 3: Parameters}

\begin{tabular}{|c|c|c|c|c|}
\hline $\begin{array}{l}\text { Simulations } \\
\text { Parameters }\end{array}$ & $\begin{array}{l}\text { Ashcraft (87) } \\
\text { Problem Size } \\
\text { Effect }\end{array}$ & $\begin{array}{l}\text { Siegler (84) } \\
\text { Addition } \\
\text { Retrieval }\end{array}$ & $\begin{array}{l}\text { Siegler (88) } \\
\text { Multiplication } \\
\text { Computation }\end{array}$ & $\begin{array}{l}\text { Lifetime } \\
\text { Simulation }\end{array}$ \\
\hline $\begin{array}{l}\text { Activation noise } \\
\text { (s) }\end{array}$ & N/A & 0.15 & 0.12 & 0.25 \\
\hline $\begin{array}{l}\text { Mismatch } \\
\text { penalty }(\mathrm{MP})^{*}\end{array}$ & N/A & $\begin{array}{l}1.5 \\
\text { (linear scale) }\end{array}$ & $\begin{array}{l}1.5 \\
\text { (linear scale) }\end{array}$ & $\begin{array}{l}1.5 \\
\text { (ratio scale) }\end{array}$ \\
\hline $\begin{array}{l}\text { Retrieval } \\
\text { threshold }(\tau)\end{array}$ & N/A & -2.25 & & -3.75 \\
\hline $\begin{array}{l}\text { Base-level decay } \\
\text { rate }(d)^{*}\end{array}$ & 0.5 & 0.5 & 0.5 & 0.5 \\
\hline $\begin{array}{l}\text { Associative } \\
\text { learning prior } \\
\text { weight (assoc)* }\end{array}$ & 1.0 & 1.0 & 1.0 & 1.0 \\
\hline $\begin{array}{l}\text { Total number of } \\
\text { problems }\end{array}$ & $\begin{array}{l}500000 \\
\text { (adults) }\end{array}$ & $\begin{array}{l}1000 \\
(4 \text { year-old) }\end{array}$ & $\begin{array}{l}150000 \\
\left(4^{\text {th }} \text { graders }\right)\end{array}$ & $\begin{array}{l}80000 \\
\text { (adults) }\end{array}$ \\
\hline $\begin{array}{l}\text { Presentation } \\
\text { frequency } \\
\text { (pbms/day) }\end{array}$ & 100 & 100 & 100 & 12 \\
\hline $\begin{array}{l}\text { Frequency ratio } \\
\text { (most/least) }\end{array}$ & 4 & 6.25 & 4 & 2.6 \\
\hline $\begin{array}{l}\text { Latency } \\
\text { intercept (I) }\end{array}$ & 0.4 & N/A & N/A & 0.2 \\
\hline $\begin{array}{l}\text { Latency of Zero } \\
\text { rule }\end{array}$ & 0.5 & N/A & N/A & 0.7 \\
\hline $\begin{array}{l}\text { Latency scale } \\
\text { factor }(F)\end{array}$ & 1.0 & N/A & N/A & 0.125 \\
\hline
\end{tabular}

* Those architectural parameters were left at their default values. 


\section{Appendix}

This appendix details the derivation of the Rehearsal Ratio Equation. Let us first derive the differential equation detailing the effect of retrievals on rehearsal frequencies using the probability rather than the odds form. If chunk $C_{l}$ had $n_{l}$ rehearsals out of a total of $n-1$ past rehearsals for both chunks, then the past probability $p_{n-1}$ of choosing $C_{1}$ is:

$$
p_{n-1}=\frac{n_{1}}{n-1}
$$

If $p^{*}$ is the current probability of retrieval of $C_{1}$ given the past rehearsal probability $p_{n-1}$, then this additional retrieval will result in a new probability of rehearsal $p_{n}$ :

$$
p_{n}=p^{*} \cdot \frac{n_{1}+1}{n}+\left(1-p^{*}\right) \cdot \frac{n_{1}}{n}
$$

The derivative of the probability of rehearsal $p$ as a function of total practice $n$ can be expressed as the difference between the successive probabilities:

$$
p^{\prime}=p_{n}-p_{n-1}
$$

which simplifies straightforwardly to:

$$
p^{\prime}=\frac{p^{*}-p}{n}
$$

A similar, though more complicated, derivation can be performed for the odds of rehearsal. The relationship is slightly different, but for most values of the activation 
noise $s$ and amount of practice $n$, it can be closely approximated by:

$r^{\prime}=\frac{r^{*}-r}{n}$

where $r$ is the past odds of rehearsal, $r^{*}$ is the current odds of retrieval, and $r$ is the derivative of $r$ as a function of $n$. Using the Dynamic Odds Equation to express the odds of retrieval as a function of the past odds of rehearsal, the differential equation becomes:

$\int \frac{d r}{r^{1 / s}-r}=\int \frac{d n}{n}$

The left-hand term of this equation can be solved by the change of variables:

$u=1-r^{\frac{s-1}{s}}$

to yield:

$-\frac{s}{s-1} \ln \left|1-r^{\frac{s-1}{s}}\right|=\ln (n)+c$

where $c$ is a constant. This equation can be transformed to extract $r$ as a function of $n$ :

$r=\left(1 \pm c n^{-\frac{s-1}{s}}\right)^{\frac{s}{s-1}}$

In the $s<1$ case, for small $s$ or large $n$, the left side of the right-hand term becomes negligible and this equation can be simplified to yield the Rehearsal Ratio Equation. 


\section{References}

Ackley, D. H., Hinton, G. E., \& Sejnowski, T. J. (1985). A learning algorithm for Boltzmann machines. Cognitive Science, 9, 147-169.

Anderson, J. A., Spoehr, K. T., \& Bennett, D. B. (1992). A study in numerical perversity: Teaching arithmetic to a neural network. In D. Levine and M. Aparcio (Eds.) Neural networks for knowledge representation, (pp. 331-335). Hillsdale, NJ: Erlbaum.

Anderson, J. R. (1990). The adaptive character of thought. Hillsdale, NJ: Erlbaum.

Anderson, J. R., \& Schooler, L. J. (1991). Reflections of the environment in memory. Psychological Science, 2, 396-408.

Anderson, J. R. (1993). Rules of the mind. Hillsdale, NJ: Erlbaum.

Anderson, J. R. (1996). Implicit memory and metacognition: Why is the glass half-full? In L. M. Reder (Ed.), Implicit memory and metacognition (pp. 123-136). Mahwah, NJ: Lawrence Erlbaum Associates.

Anderson, J. R., Bothell, D., Lebiere, C., \& Matessa, M. (1998). An integrated theory of list memory. Journal of Memory and Language, 38, 341-380.

Anderson, J. R., \& Lebiere, C. (1998). The Atomic Components of Thought. Mahwah, NJ: Erlbaum.

Anderson, J. R., Lebiere, C., Lovett, M. C., \& Reder, L. M. (in press). ACT-R: A higher-level account of processing capacity. To appear in Behavioral and Brain Sciences.

Anderson, J. R., \& Reder, L. M. (in press). The fan effect: new results and new theories. To appear in Journal of Experimental Psychology: General. 
Anderson, J. R., Reder, L. M., \& Lebiere, C. (1996). Working memory: Activation limitations on retrieval. Cognitive Psychology, 30, 221-256.

Ashcraft , M. H. (1987). Children's knowledge of simple arithmetic: A developmental model and simulation. In J. Bisanz, C. J. Brainerd, \& R. Kail (Eds.), Formal methods in developmental psychology: Progress in cognitive development research ( $\mathrm{pp}$ 302-338). New York: Springer-Verlag.

Ashcraft, M. H. (1992). Cognitive arithmetic: A review of data and theory. Cognition, 44, 75106.

Ashcraft, M H. (1995). Cognitive psychology and simple arithmetic: A review and summary for new directions. Mathematical Cognition, 1, 3-34.

Ashcraft, M. H., \& Christy, K. S. (1995). The frequency of arithmetic facts in elementary texts: Addition and multiplication in grades 1-6. Journal for Research in Mathematics Education, Vol. 26, No. 5, 396-421.

Benford, F. (1938). The law of anomalous numbers. Proceedings of American Philosophy Society, 78, 551-572.

Bobrow, D. G. (1980). Special issue on non-monotonic logic. Artificial Intelligence, Vol. 13, No. 1-2, 1-174.

Campbell, J. I. D. (1991). Conditions of error priming in number-fact retrieval. Memory and Cognition, 19, 197-209.

Campbell, J. I. D. (1995). Mechanisms of simple addition and multiplication: A modified network-interference theory and simulation. Mathematical Cognition, 1, 121-164.

Eliaser, N. M., Siegler, R. S., Campbell, J. I. D., \& Lemaire, P. (in preparation). The tie effect in simple arithmetic. 
Estes, W. K. (1964). All-or-none processes in learning and retention. American Psychologist, $19,16-25$.

Geary, D. C. (1996). The problem-size effect in mental addition: Developmental and crossnational trends. Mathematical Cognition, 2, 63-93.

Geman, S., \& Geman, D. (1984). Stochastic relaxation, Gibbs distributions, and the Bayesian restoration of images. IEEE Transactions on Pattern Analysis and Machine Intelligence, 6, 721-741.

Goldman, S. R., Pellegrino, J. W., \& Mertz, D. L. (1988). Extended practice of basic addition facts: strategy changes in learning-disabled students. In Cognition and Instruction, 5(3), 223-265.

Groen, G. J., \& Parkman, J. M. (1972). A chronometric analysis of simple addition. Psychological Review, 79, 329-343.

Hamann, M. S., \& Ashcraft, M. H. (1985). Simple and complex mental addition across development. Journal of Experimental Child Psychology, 40, 49-72.

Hamann, M. S., \& Ashcraft, M. H. (1986). Textbook presentations of the basic addition facts. Cognition \& Instruction, 3, 173-192.

Haussler, D., Kearns, M., \& Schapire, R. E. (1994). Bounds on the sample complexity of Bayesian learning using information theory and the VC dimension. Machine Learning, 14, 83-113.

Hinton, G. E., \& Sejnowsky, T. J. (1986). Learning and relearning in Boltzmann machines. In D. E. Rumelhart, J. L. McClelland, and the PDP Group, Parallel distributed processing: Explorations in the microstructure of cognition. Volume 1: Foundations (pp. 282-317), Cambridge, MA: MIT Press.

Kirk, E. P., \& Ashcraft, M. H. (1997). Verbal reports on simple arithmetic: A demanding task. Poster presented at the 38th Annual Meeting of the Psychonomic Society. 
Lebiere, C., \& Anderson, J. R. (1993). A connectionist implementation of the ACT-R production system. In Proceedings of the Fifteenth Annual Conference of the Cognitive Science Society, pp. 635-640. Hillsdale, NJ: Erlbaum.

Lebiere, C., Anderson, J. R., \& Reder, L. M. (1994). Error modeling in the ACT-R production system. In Proceedings of the Sixteenth Annual Conference of the Cognitive Science Society, pp 555-559. Hillsdale, NJ: Erlbaum.

Lebiere, C, \& Wallach, D. (in preparation). Example-based models of control problems.

LeFevre, J-A., Sadesky, G. S., \& Bisanz, J. (1996a). Selection of procedures in mental addition: Reassessing the problem size effect in adults. In Journal of Experimental Psychology: Learning, Memory and Cognition, Vol. 22, No. 1, 216-230.

LeFevre, J-A., Bisanz, J., Daley, K. E., Buffone, L., Greenham, S. L., \& Sadesky, G. S. (1996b). Multiple routes to solution of single-digit multiplication problems. In Journal of Experiment Psychology: General, Vol. 125, No. 3, 284-306.

Logan, G. D. (1988). Toward an instance theory of automatization. Psychological Review, 95, 492-527.

Lovett, M. C. (1998). Choice. In The Atomic Components of Thought, Anderson, J. R. \& Lebiere, C. (Eds.) Mahwah, NJ: Erlbaum.

Lovett, M. C., Reder, L. M., \& Lebiere, C. (1997). Modeling individual differences in a digit working memory task. In Proceedings of the Nineteenth Conference of the Cognitive Science Society, pp. 460-465. Mahwah, NJ: Erlbaum.

Lovett, M. C., Reder, L. M., \& Lebiere, C. (in press). Modeling working memory in a unified architecture: An ACT-R perspective. To appear in Miyake, A. \& Shah, P. (Eds.) Models of Working Memory: Mechanisms of Active Maintenance and Executive Control. New York: Cambridge University Press.

Luce, R. D. (1959). Individual choice behavior: a theoretical analysis. Wiley, New York, NY. 
McCloskey, M., \& Lindemann, A. M. (1992). MATHNET: Preliminary results from a distributed model of arithmetic fact retrieval. In J. I. D. Campbell (Ed.) The nature and origins of mathematicall skills, (pp. 365-409). Amsterdam: Elsevier Science.

Miller, K., Perlmutter, M., \& Keating, D. (1984). Cognitive arithmetic: Comparison of operations. Journal of Experimental Psychology: Learning, Memory, and Cognition, 10, 46-60.

Mitchell, T. M. (1997). Machine Learning. McGraw-Hill.

Newcomb, S. (1888). Note on the frequency of use of the different digits in natural numbers. American Journal of Mathematics, 4, 39-40.

Raimi, R. A. (1976). The first digit problem. American Mathematics Monthly, 83, 531-538.

Reder, L.M. (1982). Plausibility judgments vs. fact retrieval: Alternative strategies for sentence verification. Psychological Review, 89, 250-280.

Reder, L.M., (1987). Strategy selection in question answering. Cognitive Psychology, 19(1), 90138.

Reder, L.M., \& Ritter, F. (1992). What determines initial feeling of knowing? Familiarity with question terms, not with the answer. Journal of Experimental Psychology:Learning, Memory, and Cognition, 18, 435-451.

Schneider, W., \& Oliver, W. (1991). An instructable connectionist/control architecture: Using rule-based instructions to accomplish connectionist learning in a human time scale. In K. Van Lehn (Ed.), Architecture for Intelligence (pp. 113-145). Hillsdale, NJ: Erlbaum.

Schneider, W., \& Pimm-Smith, M. (1997). Consciousness as a message aware control mechanism to modulate cognitive processing. In J. Cohen and J. Schooler (Eds.), Scientific Approaches to Consciousness: 25th Carnegie Symposium on Cognition. 
Schunn, C. D., \& Anderson, J. R. (1998). Scientific discovery. In The Atomic Components of Thought, Anderson, J. R. \& Lebiere, C. (Eds.) Mahwah, NJ: Erlbaum.

Schunn, C. D., Reder, L. M., Nhouyvanisvong, A., Richards, D. R., \&Stroffolino, P.J. (1997). To calculate or not calculate: A source activation confusion (SAC) model of problemfamiliarity's role in strategy selection. Journal of Experimental Psychology: Learning, Memory, \& Cognition, 23, 1-27.

Servan-Schreiber, E. (1991). The competitive chunking theory: Models of perception, learning and memory. Ph.D. Dissertation, Carnegie Mellon University, Pittsburgh, PA.

Siegler, R. S., \& Robinson, M. (1982). The development of numerical understandings. In H. Reese \& L. P. Lipsitt (Eds.), Advances in child development and behavior (Vol. 16, pp. 241-312). New York: Academic Press.

Siegler, R. S., \& Shrager, J. (1984). A model of strategy choice. In C. Sophian (Ed.), Origins of cognitive skills (pp. 229-293). Hillsdale, NJ: Erlbaum.

Siegler, R. S. (1988). Strategy choice procedures and the development of multiplication skill. Journal of Experimental Psychology: General, 117, 258-275.

Siegler, R. S., \& Shipley, C. (1995). Variation, selection, and cognitive change. In T. J. Simon \& G. S. Halford (Eds.), Developing cognitive competence: new approaches to process modeling. Hillsdale, NJ: Erlbaum.

Van Gelder, T. (1998). The dynamical hypothesis in cognitive science. To appear in Behavioral and Brain Sciences.

West, B. J., \& Salk, J. (1987). Complexity, organization and uncertainty. European Journal of Operational Research 30, 117-128.

West, R., \& Lebiere, C. (in preparation). An ACT-R model of emergent strategies in simple games. 
Whalen, J. (1996). The influence of the semantic representations of numerals on arithmetic fact retrieval. Unpublished dissertation proposal.

Winkelman, J., \& Schmidt, J. (1974). Associative confusions in mental arithmetic. Journal of Experimental Psychology, 102, 734-736.

Zbrodoff, N. J. (1995). Why is 9+7 harder than 2+3? Strength and interference as explanations of the problem-size effect. Memory \& Cognition, 23 (6), 689-700. 